CHAPTER 9

\title{
Autonomy and Autonomy Disturbances in Self-Development and Psychopathology: Research on Motivation, Attachment, and Clinical Process
}

RICHARD M. RYAN, EDWARD L. DECI, and MAARTEN VANSTEENKISTE

\author{
INTRODUCTION 385 \\ Autonomy, Growth, and Psychopathology 385 \\ Chapter Overview 387 \\ AUTONOMOUS REGULATION AND FACILITATIVE \\ ENVIRONMENTS 387 \\ Autonomous Regulation of Behavior 387 \\ Distinguishing Autonomy From Independence 389 \\ Facilitating Environments: The Key Role of Need-Supportive \\ Socialization 390 \\ AUTONOMY AND AUTONOMY SUPPORT IN MAJOR \\ DEVELOPMENTAL PROCESSES: ATTACHMENT, \\ INTRINSIC MOTIVATION, INTERNALIZATION, \\ EMOTION REGULATION, AND IDENTITY \\ FORMATION 396 \\ Autonomy and Attachment Security 396 \\ Intrinsic Motivation: A Spontaneous Expression of Human \\ Autonomy 399 \\ Internalization: Assimilating Social Regulations and Values 403
}

\section{INTRODUCTION}

\section{Autonomy, Growth, and Psychopathology}

Autonomy, defined as self-regulation and integration in acting, is central to healthy psychological development and functioning. Classic organismic and dynamic theories have long considered the movement toward greater autonomy and integration to be a hallmark of optima development (e.g., Hartmann, 1947/1964; Jahoda, 1958; Loevinger, 1976; Piaget, 1971; Werner, 1948), a focus that continues in developmental psychologies today (see Sokol, Grouzet, \& Muller, 2013). Because of the importance of children developing capacities for autonomy and integrated behavioral regulation, support for autonomy is recognized as a central aspect of optimal parenting by many theorists (e.g., Bretherton, 1987; Grolnick, 2002; Grusec \& Goodnow, 1994; Hmel \& Pincus, 2002).

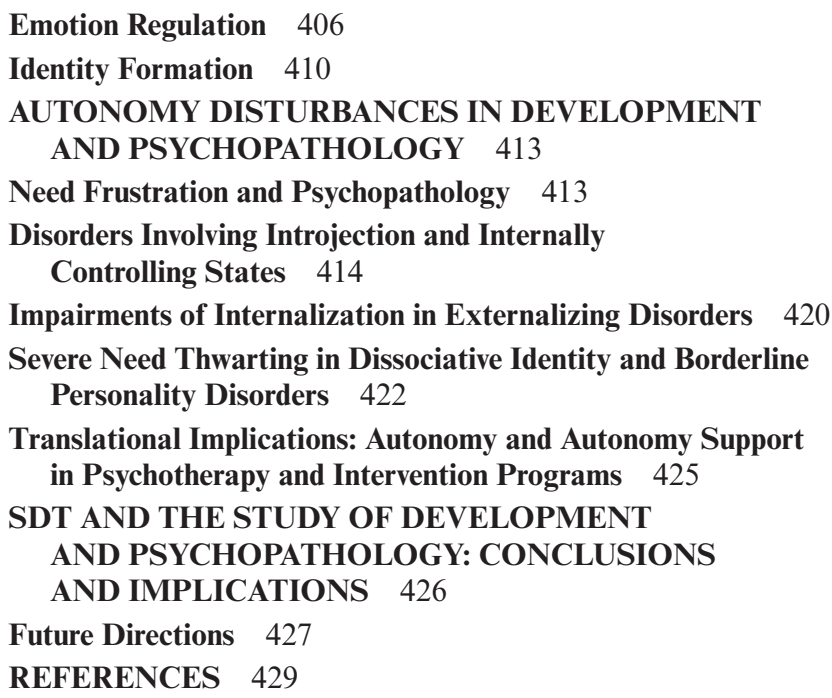

Issues of autonomy and integration are in fact frequently implicated in the development of psychopathology (Ryan, Deci, Grolnick, \& La Guardia, 2006). Historically, clinical literatures have highlighted that many forms of behavioral and mental disorders are characterized by autonomy disturbances (e.g., Shapiro, 1981; Winnicott, 1965). In many disorders, people's behaviors, emotions, and thoughts are experienced as pressured, compelled, or controlled, or alternatively uncontrollable (Ryan et al., 2006). For example, people can feel unable to regulate specific behaviors (e.g., some addictive and impulsive disorders). In other pathologies there is highly controlled, intentional behavior (e.g., restrictive eating, obsessive rituals) that is not well integrated or reflectively self-endorsed, whereas in other forms of psychopathology, usually involving considerable trauma at the hands of others, behaviors are enacted without being intentionally mediated by the self (e.g., dissociative disorders). In still others the motivation 
for pursuing intentions and goals is absent or depleted (e.g., some forms of depression). In short, compromised autonomy is entailed in a wide range of pathologies, from those involving a lack of behavior regulation to those that entail rigidly enforced self-controls and compulsive behaviors. Corresponding to this, caregivers' obstruction of children's autonomy in conjunction with genetic vulnerabilities has been shown to foster a broad range of negative effects on development and is thought to contribute to the onset of varied disorders (e.g., Bruch, 1973; Depue \& Lenzenweger, 2001; Ryan, 2005).

Given the role played by autonomy (or its absence) in both optimal development and developmental psychopathology, it is not surprising that autonomy support is emerging as an important, indeed critical, element in successful treatments for psychopathology (e.g., Mansour et al., 2012; Zuroff et al., 2007). In fact, most current behavioral and psychological intervention approaches attempt to support autonomy, albeit through different means, and with more or less explicit foci (Ryan, Lynch, Vansteenkiste, \& Deci, 2011). In some treatment approaches, support for autonomy is seen as important in its own right for building essential inner resources that can buffer vulnerabilities and enhance adjustment (e.g., Ryan \& Deci, 2008). Yet even when capacities for autonomy are not a focus, enhancing autonomous motivation for treatment enhances clinical success and engagement in behavior change over time (e.g., see Ng et al., 2012; Savard, Joussemet, Pelletier, \& Mageau, 2013; Zuroff, Koestner, \& Moskowitz, 2012).

Recognition of the importance of autonomy for healthy development and wellness has contributed to a fast-rising number of empirical studies on the topic of autonomy and its developmental dynamics. New research is coordinating phenomenological, functional, and mechanistic viewpoints in understanding autonomy and what influences it. Salient within this research is the power of social contexts (e.g., parents, teachers, coaches, peers) to facilitate the development and expression of autonomy and capacities for self-regulation in children. Salient as well is how the thwarting of autonomy, through either excessive control, conditional regard, or lack of empathy, disrupts development, and especially in vulnerable individuals, leads to dysregualtion and ill-being. Indeed, autonomy thwarting plays an important etiological role in many forms of psychopathology.

In this chapter we review the growing body of research on autonomy and autonomy disturbances primarily through the lens of Self-determination theory (SDT; Deci \& Ryan 2000; Vansteenkiste, Niemiec, \& Soenens, 2010). SDT is an empirically grounded theory informed by philosophical, clinical, and cross-cultural analyses of autonomy. SDT argues that among the predominant factors that foster resilience in development are opportunities to satisfy people's basic psychological needs, namely those for autonomy, competence, and relatedness (Ryan, 1995). Briefly, the need for autonomy refers to the necessity of experiencing a sense of volition; competence refers to the experience of effectiveness; and relatedness refers to the experience of warmth and reciprocal care. Support for the satisfaction of these needs is said to promote growth and well-being. Indeed, when social conditions support these psychological need satisfactions, individuals develop inner resources that allow them to better cope with adversity and flourish in their adult development. Interestingly, even individuals who do not explicitly value or desire these need satisfactions benefit from their fulfillment (Chen et al., in press). In contrast, the thwarting and frustration of these basic psychological needs is conducive to maladjustment and psychopathology (Ryan \& Deci, 2000; Vansteenkiste \& Ryan, 2013).

As an organismic theory, the principles informing SDT are fully consistent with a developmental psychopathology perspective (e.g., Cicchetti, 2006; Cicchetti \& Toth, 2009). In particular, SDT focuses on nonclinical, subclinical, and clinical populations, connecting the conditions conducive to optimal psychological development with depriving and thwarting conditions that redirect developmental energies into self-protection, defense, and maladaptive coping. SDT sees the pervasive experience of having basic psychological needs frustrated during development as setting the stage for later difficulties and deficits in one's capacity for self-regulation of action and experience, often through cascading negative effects (Vansteenkiste \& Ryan, 2013). In short, SDT suggests that the very same mechanisms, namely the satisfaction and frustration of people's basic psychological needs, accounts for substantial variance both in situational motivation and wellness and in developmental trajectories in self-regulation, well-being, and psychopathology.

In addition, and again in line with both developmental psychopathology (Cicchetti \& Toth, 20009) and organismic perspectives more generally, SDT sees multiple pathways to both health and illness. By identifying the resources associated with integrative tendencies and psychological resilience, SDT allows for considerable flexibility and equifinality in development (Ryan, 1993), and conversely identifies many pathways of harm leading to diverse outcomes. Conditions affecting basic psychological need satisfaction versus frustration range from biologic to cultural, and the consequences of need supports are manifest at every level of analysis, from physiological to phenomenological. SDT additionally seeks to coordinate 
these varied levels of analysis within its empirically driven framework, an aspiration that also characterizes the field of developmental psychopathology (e.g., Cicchetti, 2006; Cicchetti \& Toth, 2009).

\section{Chapter Overview}

This chapter consists of three major sections. First we define autonomy as it is classically understood and currently applied within the SDT perspective. In doing so, we not only specify what autonomy entails, but also distinguish it, both theoretically and operationally, from other developmental constructs with which autonomy is often confounded. These include the constructs of independence (Silk, Morris, Kanaya, \& Steinberg, 2003; Smetana, Campione-Barr, \& Dadis, 2004); individualism (e.g., Iyenger \& Devoe, 2003); individuation (e.g., Blos, 1979); and emotional autonomy (e.g., Steinberg \& Silverberg, 1986). Next, we elaborate on the type of social environments that are conducive to autonomous regulation via the satisfaction of individuals' psychological needs and those that have been found to obstruct and undermine autonomous regulation by eliciting need frustration.

Then we discuss the different healthy manifestations of autonomy, thereby reviewing in greater detail its developmental origins. Using research from both SDT and attachment theory, we begin by highlighting that secure attachments in childhood are developed through autonomy supportive and involved caregivers, who not only hold and protect (i.e., provide security) but also stimulate the budding initiative and self-expression of the infant (i.e., support autonomy), setting the stage for developing further capacities for mature self-regulation (e.g., Miklikowska, Duriez, \& Soenens, 2011; Whipple, Bernier, \& Mageau, 2009). Apart from being conducive to secure attachments, autonomy supportive relationships support the integrative functions of the self that allow for greater internal psychological coherence and more effective behaviora regulation. Exemplifying this, we then turn to evidence concerning how autonomy supportive contexts facilitate developmentally relevant integrative processes including: (1) intrinsic motivation; (2) internalization and integration of social regulations; (3) the development of emotion regulation; and (4) the formation of identity. We pay particular attention to how socializers, especially parents, either facilitate or thwart the developing child's autonomy, thereby supporting or hindering the individual's inner resources for successfully negotiating each of these developmental lines.

These reviews of autonomy in psychological development, and of parental nurturance versus thwarting of autonomy, provide the foundation for examining autonomy disturbances in varied types and presentations of psychopathology. The next section focuses on psychopathology is twofold: we consider autonomy depriving and thwarting environments as an etiological factor in psychopathology; and we highlight how autonomy frustrations are often a symptomatic factor in various disorders and sometimes a by-product of other cascading effects. Moreover, we identify three general types of psychopathology in which autonomy is differently implicated. These are internally controlling disorders, in which there is excessive self-control or internal compulsion; impairments of internalization, reflecting both impoverished development of self-regulation capacities or lack of value for social regulations; and finally fragmented self-functioning, which we relate to chronic or traumatic need thwarting. We conclude this section by considering implications not only for clinical interventions, but also for the design of social institutions such as schools and workplaces that can support persons vulnerable to psychopathology. Finally, we provide a summarizing overview and discuss a number of critical future research directions.

\section{AUTONOMOUS REGULATION AND FACILITATIVE ENVIRONMENTS}

\section{Autonomous Regulation of Behavior}

Before proceeding to the intricacies of autonomy in development we begin with some basic definitions and distinctions. The term autonomy is derived from the Greek words autos (self) and nomos (rule). Autonomous actions are thus those that are self-ruled or governed by the self. The opposite of autonomy is heteronomy, when one's behavior is regulated or ruled by forces experienced as heteron or alien to one's sense of self.

Phenomenological analyses suggest that, when people act autonomously, they feel a sense of ownership of their actions (Pfander, 1967/1908). When autonomous, people are willingly engaged in activities. Accordingly, their actions are characterized by an internal perceived locus of causality (de Charms, 1968; Ryan \& Connell, 1989). This quality of self-endorsement indicates that autonomous actions are informed by, and reflective of, individuals' abiding preferences and values and are congruent rather than discordant with their sense of self (Ryan Legate, Niemiec, \& Deci, 2012; Shapiro, 1981; Weinstein, Prybylski, \& Ryan, 2013). In contrast, when behaviors are heteronomous they are experienced as controlled. The actor experiences a sense of external or internal control, compulsion, or pressure to enact the behavior (Deci \& Ryan, 2000). In attribution terms, people have an external 
perceived locus of causality with respect to controlled behaviors. Interestingly, the functional effects of autonomy can be detected from early development and a sense of initiative and ownership of action is apparent from early infancy (Stern, 1985).

Although the concept of autonomy refers to integrated regulation by the self, autonomy is nonetheless a relative concept insofar as actions vary in their degree of autonomy. Autonomous functioning reflects the extent to which ongoing behaviors, thoughts, and feelings match with one's interests and values. Since most actions are both multiply motivated and more or less congruent, autonomy is inevitably a dimensional concept, and differentially associated with different types of motives. It is therefore basic to our view that autonomy is a quality of behavioral regulation, which is characterized by and requires an open processing of possibilities such that ongoing behaviors and underlying experiences are maximally integrated and volitional. This quality of behavioral regulation is relevant across the life span, even as the contents and focus of activities and values change with development

Phenomenological analysis also points to the relationship between autonomy and integration in functioning. When autonomous, people are wholeheartedly behind their actions, and this is reflected in the coherence and lack of internal conflict involved in those actions. Yet to varying degrees behavioral regulations can represent compartmentalized or defended motives, or can be enacted because of external or internal pressures without reflective ascent or full endorsement. Thus the experience of autonomy is directly related to the relative integration of one's motivations and valuing (Ryan \& Deci, 2008).

Although SDT's definition and understanding of autonomy is relatively unique within empirical psychology, it is strongly informed by philosophical treatments of the topic (e.g., Dworkin, 1968; Friedman, 2003). It can also be related to psychodynamic (e.g., Schafer, 1968; Loevinger, 1976; Shapiro, 1981) and humanistic (e.g., Rogers, 1963) traditions that have considered autonomy and integration to be highly important. Whether addressed in terms of self-endorsement, congruence, self-actualization, authenticity, will, or operating from the true self (see Ryan et al., 2006) many of these prior thinkers were addressing a shared idea, which is termed autonomy within SDT.

In addition, both the underlying sensibilities (e.g., recognition of values or interests in acting) and abilities (e.g., reflective capacities, goal selection, self-regulation skills) entailed in autonomy must develop, and accordingly there are individual differences in autonomous functioning (Deci \& Ryan, 1985a; Weinstein, Przybylski, \& Ryan, 2012). Yet autonomy is also situational, in part because social contexts differ in the extent to which they are autonomy supportive, controlling, or amotivating. This is true at all levels of development as parents, teachers, coaches, managers, and spouses for example, can all facilitate or stifle autonomy in an individual, with significant mental health consequences. Yet whether due to dispositional or proximal supports, people evidence fuller functioning and more positive behavioral and psychological outcomes when more autonomous, including more sustained persistence, enhanced creativity, and higher quality performance (Vansteenkiste \& Ryan, 2013).

Although phenomenologically autonomy is characterized by the experience of actions as being self-organized and volitionally supported, in terms of the quality of behavior, autonomy is manifest at varied levels of analysis. For example, evidence is emerging concerning the distinct neural basis of autonomous functioning relative to controlled, nonautonomous, motivational processes (e.g., Lee \& Reeve, 2013; Murayama et al., 2014). In general terms, autonomy depends on the coordination between prefrontal cortical regions and subcortical striatal-thalamic areas that promote or inhibit motivation as well as on inputs from the hippocampus and amygdala that can provide contextual and affective information (e.g., Bradley, 2000; Chambers, Taylor, \& Potenza, 2003). Autonomy requires supervisory and selective functions that are fully informed by affective and memory related processes (Di Domenico, Fournier, Ayaz, \& Ruocco, 2013; Ryan, Kuhl, \& Deci, 1997). Impairment in the development of prefrontal areas and interconnections with limbic structures produces vulnerability to autonomy disturbance (e.g., Bechara, Tranel, Damasio, \& Damasio, 1996)

Interestingly, under autonomy-supportive conditions people appear more capable to recruit and use selfregulation strategies associated with executive functioning, a fact with important implications for development. For example, Bernier, Carlson, and Whipple (2010) examined whether maternal sensitivity and autonomy support were related to toddlers' capacity for executive control, which was assessed via a set of cognitive indicators involving flexible goal setting, impulse control, and planning. After controlling for children's cognitive capacity and maternal education, both sensitivity and maternal autonomy support, assessed when children were 12-15 months old, related to executive control at 18 and 26 months, with maternal autonomy support emerging as the most consistent predictor of enhanced executive control.

In fact, exposure to particular social contexts shapes the use of neurological resources required for autonomous functioning. For example, Di Domenico et al. (2013) argued, based on SDT, that people's abilities to develop 
and act from a coherent sense of self are facilitated by satisfaction of the basic psychological needs. Using functional near-infrared spectroscopy (fNIRS), they examined the effect of need satisfaction on activity in the medial prefrontal cortex (MPFC), an important brain region for integrative processing of information about the self (Ryan et al., 1997). Participants engaged in a task involving a series of forced choices expressing personal preferences. Decisional conflict (i.e., choice difficulty) between the response options was manipulated using participants' previously collected preference ratings. Findings revealed that need satisfaction predicted elevated MPFC activity during high-conflict situations, suggesting that need satisfaction may facilitate access to and utilization of self-knowledge.

To summarize, autonomy is integrated self-regulation, and it is accompanied by a phenomenological experience of self-organization and volition, which is described in attribution terms by an internal perceived locus of causality (Deci \& Ryan, 2000). Autonomy thus involves an experience of regulation by the self, and it is contrasted with actions experienced as pressured, controlled, or caused by sources external to the self. These later experiences are central to many forms of psychopathology, and can increasingly be mapped to neurological underpinnings.

\section{Distinguishing Autonomy From Independence}

An important distinction, particularly relevant to development, is that between autonomy and independence. Although autonomy and independence have been clearly distinguished in philosophical analyses, theorists in developmental psychology have often equated or conflated these concepts (e.g., Silk et al., 2003). Dependence is reliance on others for resources or guidance, so independence is not relying on others. Autonomy, by contrast, connotes willingness or volition. Given this, in the SDT view individuals can be autonomously dependent or autonomously independent, depending on circumstances. They can also sometimes be controlled or pressured into dependence, as when they feel forced to rely on others because of illness or financial hardship, or they can be heteronomously independent, as when pushed away from relying on others, who are unavailable or unwilling to provide assistance.

SDT suggests that autonomous forms of dependence (for example, when a teenager willingly turns to parents for guidance) are often quite beneficial, a view that stands in contrast to some developmental perspectives that have equated autonomy with separation or independence (Ryan \& Lynch, 1989). For instance, Blos (1979) portrayed normative development in terms of an increasing relinquishing of both dependency on, and attachment to, parents.
He viewed moving away from parental supports as indicative of increasing individuation. Based on Blos, Steinberg, and Silverberg (1986) developed a construct labeled emotional autonomy that was intended to be a marker of individuation. Scale items assessing emotional autonomy made reference to "not using parents as resources," "disagreeing with parents," and "being different when not with parents."

Working from the SDT lens, Ryan and Lynch (1989) reexamined the emotional autonomy scale, suggesting that it captured a detached relationship with parents rather than autonomy and they argued that such detachment or separation from parents is neither a necessary nor ideal pathway of development. They also suggested that such detachment might be more likely in adolescents who did not have autonomy-supportive parents. They therefore predicted that emotional autonomy as thus measured rather than being indicative of positive maturation, would predict poorer adjustment. Consistent with this reasoning, findings indicated that adolescents high in emotional autonomy were those feeling less accepted by their parents and less lovable. These teens also evidenced poorer wellness outcomes overall. Ryan and Lynch (1989) interpreted these findings as highlighting the need to differentiate independence or nonreliance from autonomy.

Extending SDT's distinction between autonomy and independence, Van Petegem, Beyers, Vansteenkiste, and Soenens (2012) directly examined adolescents' level of independence, as well as their motives for both independent and dependent functioning. They found that autonomous (i.e., volitional) and controlled (i.e., pressured) motives for both independence and dependence played a more significant role in predicting adolescents' psychosocial functioning than did their level of independence as such. More autonomous forms of motivation for either dependence or independence were strongly associated with greater well-being and less problem behavior, whereas the level of independence was less predictive of these outcomes.

Because of the widespread interest within the developmental literature in assessments of autonomy and independence, Van Petegem, Vansteenkiste, and Beyers (2013) undertook a comprehensive study of 15 prevailing measures in adolescent psychology relevant to these constructs. Using higher order factor analyses, Van Petegem et al. (2013) showed that two basic dimensions provided the most parsimonious summary of the existing heterogeneity across available measures. A first dimension denoted the degree of distance versus proximity in the parentchild relation; a second dimension represented volition versus pressure in the relationship. The crossing of these two dimensions gives support for SDT's distinction between autonomy and independence. For example, the 
emotional autonomy scale (Steinberg \& Silverberg, 1986), characterized by Ryan and Lynch (1989) as reflecting more detachment than autonomy, fell in the quadrant tapping pressured distance. In contrast, a scale of emotional reliance (Ryan, La Guardia, Solky-Butzel, Chirkov, \& Kim, 2005), tapping adolescents' willing reliance on parents, fell in the volitional proximity-seeking quadrant. Van Petegem et al. (2013) further showed that the dimension of distance (versus proximity) was positively related to both avoidance and anxiety-based forms of attachment, whereas the dimension of volition (versus pressure) was negatively related to these insecure attachment styles. Such studies thus support the SDT literature in strongly distinguishing, both theoretically and empirically, autonomy from independence.

The distinction between autonomy and independence is critical to developmental psychology not only in childhood and adolescence, but also across the life span. For example, with advanced age people inevitably become more reliant or dependent on others. Nonetheless, there are important differences in wellness outcomes as a function of whether an older person's reliance on others feels autonomous and volitional or forced upon them (e.g., V. Kasser \& Ryan, 1999; Vallerand, O'Conner, \& Hamel, 1995). When elderly individuals can feel volitionally dependent and still experience a sense of choice in everyday living they remain more vital and resilient.

This distinction is also critical to cross-cultural psychologies. Various scholars have suggested that, because collectivist cultures emphasize duty and obligation to the group, autonomy is not very important for their members (e.g., Iyengar \& Devoe, 2003; Markus, Kitayama, \& Heiman, 1996). Yet this is a misinterpretation of the concept of autonomy, as it assumes people cannot autonomously engage in a duty, willingly comply with their parents' choices, or, with full volition, adhere to collectivistic cultural norms. In a direct examination of the distinction between autonomy and independence in the cross-cultural area, Chen, Vansteenkiste, Beyers, Soenens, and Van Petegem (2013) demonstrated that both Chinese adolescents' volitional reliance on parents as well as their volitional independent functioning yielded unique well-being benefits. Overall then, considerable data suggest that across cultural contexts, support for autonomy matters in both the internalization of ambient practices and in fostering individuals' overall wellbeing (Chirkov, Ryan, \& Sheldon, 2010)

Finally, it should be noted that the concepts of independence and autonomy yield a different relation to individuals' development of relatedness and intimacy. Excessive strivings for independence often come at the cost of the development of trustful and warm relations, suggesting that, for individuals to develop most optimally, they need to balance their strivings for independence and relatedness. Yet, from the SDT perspective, the experience of volition and autonomy does not necessarily stand in conflict with the development of warm relations, because as one volitionally relies on another, a sense of intimacy can be maintained without giving up one's autonomy. Thus, instead of autonomy development extracting a price in terms of increased relational distance or tension, in SDT's view autonomy and relatedness optimally stand in a mutually reinforcing relation to each other (Hodgins, Koestner, \& Duncan, 1996; LaGuardia, Ryan, Couchman, \& Deci, 2000). Indeed, parental support for children's autonomy enhances parent-child relatedness, a pattern that persists across the age-related changes in types and foci of dependencies.

\section{Facilitating Environments: The Key Role of Need-Supportive Socialization}

As an organismic theory, SDT endorses a transactionalecological conceptualization of development (e.g., Cicchetti \& Valentino, 2006), in which growth and competencies, as well as impairments and liabilities at earlier stages of development impact the direction and quality subsequent development. Within such an organismic model a critical issue concerns the necessary nutrients for optimal development, which for SDT are represented by the satisfaction of the needs for autonomy, competence, and relatedness (Ryan, 1995). That is, individuals are prepared to optimally develop psychologically and socially when their caregiving environments support these basic needs. When these needs are thwarted, people are prepared with other protective, even defensive, responses, and both the healthier and the more defensive processes can have cascading developmental consequences (Vansteenkiste \& Ryan, 2013).

Already in the earliest SDT parenting research (Grolnick \& Ryan, 1989), three distinct parenting dimensions were identified as being critical for satisfying children's basic psychological needs and thus fostering more integrative development and wellness (Grolnick \& Pomerantz, 2009; Soenens \& Vansteenkiste, 2010). Specifically, these dimensions are parental autonomy support, parental structure, and parental involvement. Environments that supply these three core nutrients for basic psychological need satisfactions energize healthy self-development. SDT's focus on the satisfaction of children's psychological needs and facilitation of intrinsic growth tendencies is relatively unique in the prevailing parenting literature because few models are clear about the exact processes 
that underlying children's growth. At the same time, SDT maintains that the very blocking and direct thwarting of these needs not only prevents children from growing but also can even elicit maladjustment and psychopathology. Children's psychological needs will get more readily frustrated when parents are controlling, chaotic, and neglectful. In what follows we discuss the SDT model of parenting in detail, comparing it with alternative perspectives and contrasting constructs.

\section{Parental Autonomy Support and Control}

Being autonomy supportive literally means that one person is supporting the other's self-management or self-rule. Research has identified a number of elements in interpersonal interactions that enhance experiences of autonomy. Most important, in being autonomy supportive a parent (or other caregiver) attempts to understand and acknowledge the child's perspective. Critical to taking the children's frame of reference is an authentic curiosity from the side of the caregiver, who is sincerely interested in what is going on for the child. When this occurs, one is in a position to be responsive to whatever issues are salient. In addition, autonomy support entails using minimal controls to foster behavior, and instead providing a meaningful rationale for behavioral requests and limits where possible to help anchor internalized motivation. Autonomy support requires respecting the children's pace of development, so demands can be volitionally and effectively engaged, and providing opportunities for choice and voice when possible, all facilitate autonomy. In contrast, being controlling involves parents pressuring the children to do what they want or value, emphasizing obedience and compliance (Grolnick, 2002; Soenens \& Vansteenkiste, 2010) and, in more extreme contexts, using punishments and physical threats. Dozens of studies have convincingly shown that autonomy-supportive parenting yields various benefits, including greater teacher-rated competence and grades (e.g., Grolnick \& Ryan, 1989), more personal well-being (e.g., Chirkov \& Ryan, 2001; Lekes, Gingras, Philippe, Koestner, \& Fang, 2010), and better social adjustment (e.g., Soenens \& Vansteenkiste, 2005).

Importantly, much like the experience of autonomy cannot be equated with independent functioning, the support of autonomy in SDT does not denote the promotion of independence. This was demonstrated in four studies by Soenens and colleagues (2007), who showed that parental promotion of independence and parenta autonomy support were only moderately positively correlated. Moreover, while both independence promotion and autonomy support were positively associated with psychosocial functioning at the correlational level, when the two competed for variance in predicting these outcomes, only autonomy support was a significant predictor. In other words, the aspect of parenting accounting for better outcomes was autonomy support rather than the promotion of nonreliance. Soenens, Vansteenkiste, and Sierens (2009) subsequently showed that adolescents who perceived parents as allowing volitional dependence reported better psychosocial functioning than those who felt parental pressure toward either independence or dependence.

Rather than working from their children's perspectives, controlling socializing agents alter, minimize, or deny the children's perspectives, thereby projecting their own wishes and standards upon the children and pushing the children in the parent-desired directions. SDT's formulation of controlling parenting can be further differentiated into two categories (Soenens \& Vansteenkiste, 2010). Parent can be controlling using externally controlling methods, such as the reliance on controlling reward or punishment contingencies. Externally controlling methods can be explicitly stated, but often the controlling contingencies are implicit and learned over time. A second subtype of controlling parental practices is internally controlling practices. This style of parenting has some overlap with psychological control as discussed in the frameworks of Schaefer (1959), Barber (1996), and Steinberg (2005). In this case, parents attempt to instill in the child positive and negative self-related feelings contingent upon compliance with parental standards and goals.

Internally controlling parental practices have been a concerted focus of research within SDT, especially through work on parental conditional regard. Parental conditional regard (PCR) describes parents or other caregivers using their attention, affection, and love to control their children's behavior (Assor, Roth, \& Deci, 2004). As a socializing method, PCR is a widely used and sometimes advocated parenting practice (e.g., Sears, Maccoby \& Levin, 1957; Fu \& Markus, 2014). Yet from the SDT perspective PCR is theorized to be controlling and therefore to have both subtle and more manifest detrimental effects on self-functioning. Indeed, parents who use conditional regard are essentially pitting the children's needs for relatedness and autonomy against each other. The message conveyed is essentially "to get my love you need to give up your autonomy." To maintain positive relatedness, the children have to comply, irrespective of the fit of the parental demands with their own developing interests, skills, or values. Yet, even when complying, children who experience PCR do not feel accepted but instead evidence resentment, because what is conveyed is that they are not being accepted for who they are but instead for whether they live up to their parents' demands. As such, the use 
of PCR with children serves to thwart the needs for both relatedness and autonomy, with resulting decrements in adjustment and well-being (e.g., Assor, et al., 2004; Roth \& Assor, 2012).

\section{Parental Structure and Parental Chaos}

Apart from parental autonomy support, SDT also highlights the critical role of parental structure, which concerns the degree to which parents or other caregivers create the necessary conditions for children to begin developing a sense of mastery and provide ongoing assistance to optimize competence development (Farkas \& Grolnick, 2010; Grolnick \& Pomerantz, 2009; Reeve \& Halusic, 2009). Structure entails the provision of an environment that makes optimal demands on children's expanding skills. Structuring parents provide guidance and scaffolding upon which the unfolding processes of development can climb. Specifically, provision of structure by parents includes: helping to adjust environments so that they can be mastered by the children without excessive anxiety; conveying consistent and clear expectations; providing guidance about the contingencies between behaviors and outcomes; offering meaningful informational feedback as the children engage in activities; and other elements of support focused on promoting the children's feeling of effectance in acting. In the research by Grolnick and Ryan (1989), parental structure predicted children's understanding the sources of control over outcomes in their lives, enhancing both the children's sense of predictability, and of competence or efficacy. In the educational domain, Mouratidis, Michou, Vansteenkiste, and Lens (2013) showed that competence need satisfaction largely accounted for the relation between perceived teacher structure and children's wellness and use of self-regulation strategies.

Although structure aids the building of competence, this process might be more or less facilitated depending on whether an autonomy-supportive or controlling style is used when introducing and maintaining the structure (Soenens \& Vansteenkiste, 2010). Specifically, the competence-enhancing impact of structure is likely strengthened when the structure is communicated in an autonomy-supportive way and diminished when done in a controlling way.

The differential impact of providing structure in autonomy supportive versus controlling ways was demonstrated early within SDT research, most notably in an experimental study by Koestner, Ryan, Bernieri, and Holt (1984). In a school setting first- and second-grade children came individually to an art room to do a painting project.
An experimenter set limits on their behaviors, advocating neatness and organization, either in a controlling or autonomy-supportive way. The autonomy-supportive method was based on SDT and included a rationale, an expression of empathy, and a clear limit. The controlling method simply directed the child to follow the limit. It was found that the controlling limits undermined the enjoyment of painting and creativity relative to autonomysupportive limits.

Overall then, structure is in no way antithetical to support for autonomy; on the contrary, the two often go hand in hand (Stipek \& Seal, 2001). Yet this conceptual point can easily be lost if parental structure is described as, or equated with, parental control. That is, if parental structure involves parents exerting pressure and control over children's functioning, that parental structure would indeed stand in contradiction to parents' encouragement of children's volitional functioning. Similarly, if parents are permissive, granting unmitigated freedom rather than specifically supporting autonomy, this would be contrary to providing structure. Parental autonomy support, in fact, can and optimally does involve parents introducing and maintaining structure, and does not entail avoiding all guidance and scaffolding (Grolnick \& Pomerantz, 2009; Soenens \& Vansteenkiste, 2010). SDT also expects that structure can be imposed in controlling ways, with less beneficial outcomes. Thus, the concepts of autonomy support and structure allow for a clearer differentiation between critical dimensions needed in parenting.

Consistent with this reasoning, Jang, Reeve, and Deci (2010) showed that structure and autonomy support were modestly positively (rather than negatively) correlated and independently contributed to positive educational outcomes. Along similar lines, examining a broad set of components of parental structure, Farkas and Grolnick (2010) found parental structure and autonomy support to yield unique positive associations with seventh- and eighth-grade children's perceived competence and actual grades. Overall, then, past research has found structure and autonomy support to be positively related to each other rather than antagonistic, and often each has yielded unique associations with a host of different outcomes. Moreover, by distinguishing the two concepts, one can assess the meaningful combinations between them, with expectations that controlling structure will have negative effects on development, whereas autonomy-supportive structure will be more positive. In fact, a number of studies in the domains of education (e.g., Vansteenkiste et al., 2012) and sports (e.g., Curran, Hill, \& Niemiec, 2013) indicate that 
the most optimal outcomes are attained under conditions of high autonomy support and high structure.

\section{Parental Involvement and Parental Neglect}

SDT also suggests that more than structure and autonomy support are needed to facilitate development. In addition, involvement, especially autonomy-supportive involvement is also helpful. Involvement refers to a parent's dedication of attention and caring, which both supports the child directly and conveys relatedness and connection as he or she faces developmental challenges (Grolnick \& Ryan, 1989). Parental involvement has long been found to have positive effects on children's behavioral regulation and well-being (e.g., Baldwin, 1955). In the Grolnick and Ryan (1989) study, assessment of involvement included the time parents spent with their children in interactive ways, their knowledge of their children's day-to-day life, and the warm tone of interpersonal contact. Parental involvement and in particular the mothers' involvement predicted children's achievement and grades in school, and was negatively correlated with teachers' ratings of children's acting out behaviors. Subsequently, Grolnick, Ryan, and Deci (1991) found that parental involvement, along with autonomy support, predicted children having greater inner resources for school engagement, especially greater feelings of autonomy and competence. Further, Cheung and Pomerantz (2011) found that in both U.S. and Chinese samples, involvement predicted engagement and achievement in schools. In both nations, when such involvement was accompanied by autonomy support children also had greater perceptions of competence and better emotional functioning.

More recently, Grolnick (in press) looked at parents motivation for their involvement with children. This is a particularly interesting area of investigation because parents can be involved both out of controlling motivations such as introjection or external pressures or because of value and interest in their children. Grolnick found that the more autonomous the parental motivation, the higher the level of involvement. Moreover, identified regulation was especially predictive of child outcomes one would expect from such willing involvement, such as more positive academic self-concept and better school grades.

These and related findings speak to the ways parents are involved, which can vary considerably. For example, much like the dynamic involved in conditional regard, parents can make their involvement (i.e., their devotion of time and resources to the child) dependent on obedient behavior, which SDT suggests will come with a cost. Consistent with this idea, Weiss and Grolnick (1991) studied the relations of parental involvement and autonomy support to adolescents' internalizing and externalizing symptoms. Adolescents rated their mothers and fathers on dimensions of involvement and autonomy support and completed a self-report profile of symptoms. Analyses indicated that parents who were perceived to be both highly involved and autonomy supportive had children who reported very low levels of either internalizing or externalizing symptoms. Yet there were significant interactions between perceived parental involvement and autonomy support on both types of symptoms, indicating that the combination of high involvement and low autonomy support yielded a high level of symptoms.

This general pattern of findings has been observed in other studies. Wouters, Doumen, Germeys, Colpin, and Verschueren (2013) found that high parental responsiveness strengthened the relations between psychologically controlling parenting and contingent self-esteem in university students. Aunola and Nurmi (2004) found in a three-year longitudinal study among 5-6-year-old children that parents' exertion of controlling practices slowed children's progress in mathematics, a trend that was exacerbated in cases where parents displayed high affection. Interestingly, the combination of high parental control and responsiveness appeared not only to yield a personal well-being cost but also to generalize to young adults' empathic responses. In this context, Kanat-Maymon and Assor (2010) showed that parental control related positively to the child perceived distress when their romantic partners were in need, whereas parental control was negatively related to empathic responding as reported by the partners, both effects being strengthened when parents were experienced as highly responsive.

It thus appears that feeling close to very controlling parents can be detrimental to a developing child's personal and social well-being, presumably because these children's psychological needs for autonomy and relatedness are insufficiently met. That is due to the high yet controlling involvement of parents, which may leave the children feeling as if they have no other choice than to connect with their parents by complying with their demands. Similar to the dynamics characterized by enmeshed families, however, the children's connections are likely to be fueled with feelings of internal conflict and pressured loyalty, thereby causing heightened emotionality and self-preoccupation, which may interfere with their capacities to respond empathically to others (Kanat-Maymon \& Assor, 2010). In short, the combination of high parental control and high 
responsiveness may create confusion because parents are sending conflicting messages to the their children (Aunola \& Nurmi, 2005).

\section{Parent's Psychological Needs: Contextual and Reciprocal Influences}

Thus far, our focus has been on how parents affect children's development and problem behavior through the nurturance or undermining of children's psychological needs. Yet parents themselves are subject to influences that can either support or thwart their capacities to support their children's development. That is, to the extent that parents engage in need-satisfying activities in their own lives, they are likely to have more energy available to be involved with their children, thereby being better attuned to the children's perspectives and, hence, being more capable of supporting children's unfolding interests, values, and skill development. To the extent that parents' psychological needs get thwarted, their energy levels would be more readily eroded so they would become more self-centered rather than open and receptive for their children's concerns and interests. Such self-centered preoccupation may either manifest through a direct withdrawal and involvement from the children or, to the extent that they are involved, parents would be more easily enforcing their own viewpoint or be unpredictable and inconsequent. This reasoning is graphically displayed in Figure 9.1, in which parents own need satisfaction and need frustration are part of the proximal determinants of their approach to their children. In an examination of this dynamic, De Haan, Soenens, Dekovic, and Prinzie (2013) found need satisfaction as reported by the parents in the parent-child relationships relate negatively to the adolescents' reports of negative parental styles (e.g., overreactive or controlling).

At the same time, a variety of more distal factors, either pressuring or facilitative, will impact on parents' experiences of need satisfaction and need frustration. Pressures on parents can come from their children's behaviors in the interactions of the parents and children. That is, there are both reciprocal influences from the children and proximal and distal social and economic pressures on parents from other sources that impact their resources (Deci \& Ryan, 2012). For example, research on child temperament suggests that children with difficult temperaments may make it harder for parents to refrain from controlling behaviors. Within the SDT literature, Grolnick, Weiss, McKenzie, and Wrightman (1996) found that parents who perceived their adolescents to be more difficult in temperament were also more controlling. In addition, it has been found that when children evidence poorer performance or get poorer grades in schools, parents often become more controlling (Aunola \& Nurmi, 2005; Grolnick, Gurland, DeCourcey, \& Jacob, 2002). In a particularly interesting study, Pomerantz and Eaton (2001) found that a child's low achievement was

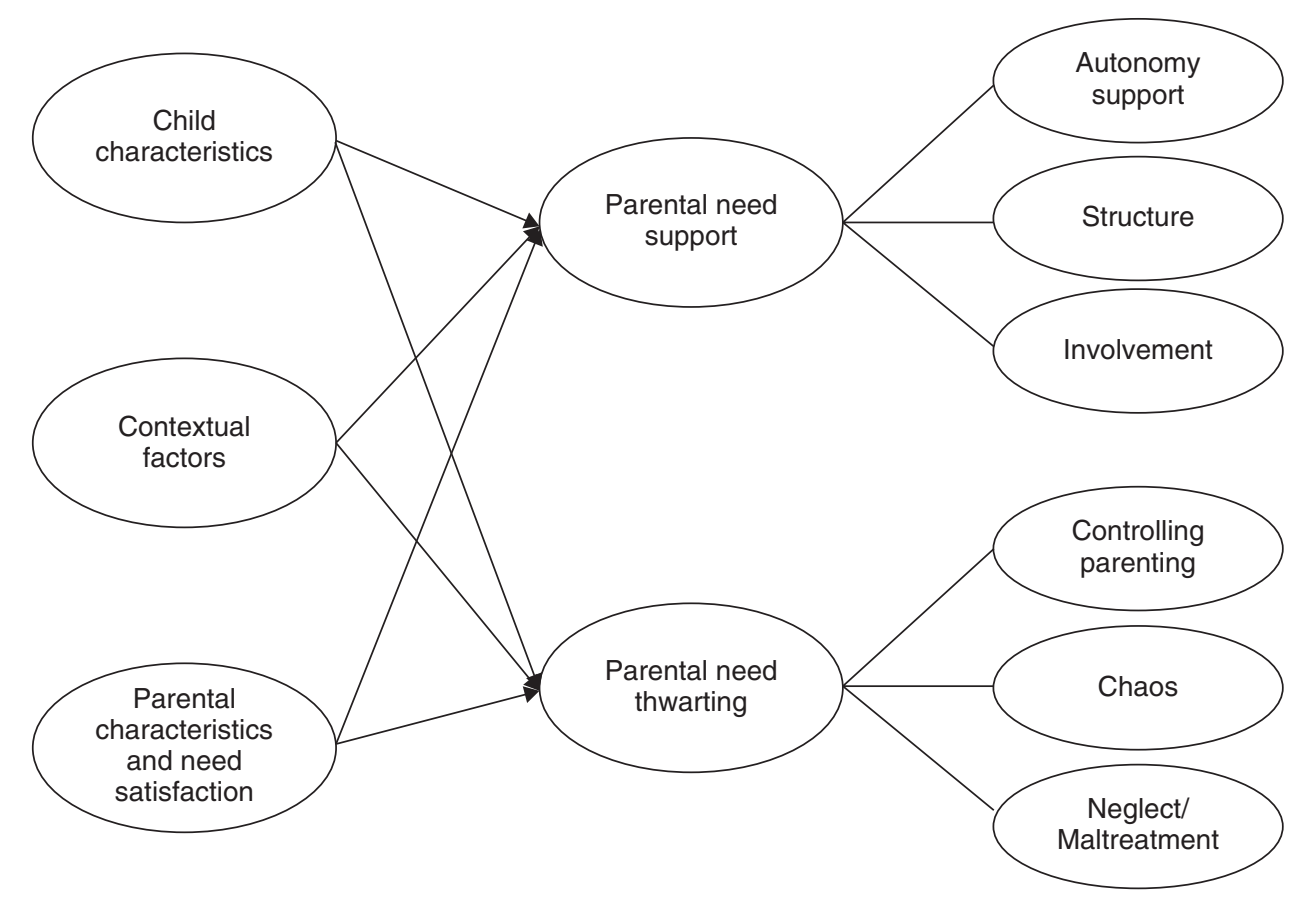

Figure 9.1 Graphic overview of the role of antecedents of parental need support and need thwarting. 
associated with increased maternal worry, and worry predicted more controlling behavior, which in turn negatively affected the child's achievement. Similarly, adolescents' defiance against parents' moral rules elicited a more controlling parental approach over time (Vansteenkiste, Soenens, Van Petegem, \& Duriez, 2014).

The parents' own supports can also bear on their capacity to nurture their children. For instance, Landry et al. (2008) showed that mothers who experienced greater social and partner supports were more able to trust the developmental process and thus support their children's autonomy. Similarly, Bouchard, Lee, Asgary, and Pelletier (2007) found that fathers' involvement and satisfaction in parenting was related, not only to their own perceived competence, but also to their perceptions that their partners placed confidence in their parenting abilities. In addition, Assor et al. (2004) found that many parents display the same parenting approaches (e.g., conditional regard) they were exposed to as children, thus exhibiting the intergenerational transmission of parenting styles.

Also parents often face a host of contextual pressures, which lead them to adopt a more controlling approach toward their children. Economic strain (Kasser, Ryan, Zax, \& Sameroff, 1995), stressful life events (Grolnick, Bridges, \& Connell, 1996), and experimentally induced stress (Grolnick et al., 2002) have all been associated with controlling parenting behavior. In one illustrative experimental study, Grolnick, Price, Beiswenger, and Sauck (2007) examined the effects of situational pressures on mothers' autonomy support, relative to control, in the social domain. The authors had 60 mothers work on a laboratory task with their fourth-grade children, presumably in preparation for meeting new children. Mothers in an evaluation condition were told that their children would be evaluated by other children and that based on this, the researchers would determine how much each child was liked and accepted by their peers. In the no-evaluation condition, mothers received no mention of peer evaluation. Results revealed that mothers in the evaluation condition spent more time giving answers to their children, especially mothers with more controlling parenting attitudes, and they also exhibited more controlling behaviors. In addition, mothers with high social contingent-self-worth who were in the evaluation condition exhibited the most controlling behaviors.

Apart from contextual pressures, internal forms of pressure, such as anxieties and worry, have also been associated with more controlling parent behaviors, especially when the worries concern their children. Gurland and Grolnick (2005), for example, examined mothers' perceptions of environmental threat regarding such issues as future economic instability and resource scarcity. Perceptions of future threat were positively associated with more controlling behaviors exhibited during parent-child tasks. In turn, these controlling behaviors were negatively associated with children's volitional motivation. Moreover, the relation between perceived threat and children's motivation was mediated by controlling parenting.

As can be noticed in Figure 9.1, parents' own personality functioning may also lead them to rely on certain parenting strategies more than others. Critical in this respect is parents' organismic trust in the development of their children (Landry et al., 2008), with parents high in organismic trust being more relaxed in their expectations regarding milestones and engaging in fewer social comparisons. Landry and colleagues showed that such organismic trust led mothers to rely on different parenting strategies during a play session where they and their 12-13-month-old infants were given problems to solve together. Mothers' autonomy support and structure were assessed using ratings of the extent to which the mothers took the children's perspectives, followed the children's pace, and structured the situation to be fitting with the children's skills. Autonomy thwarting behaviors were also measured, including tallying empathic failures and interfering in the children's pace, among other indicators. Results revealed that organismic trust, even when controlling for parental income and education and children's temperament and level of development, was significantly associated with greater autonomy support as opposed to autonomy thwarting.

Other personality factors within parents are also relevant. For example, Deci and Ryan (1985a) early on reported that persons with a stronger control orientation were less prone to support autonomy in others. The reason that parents with a stronger controlled orientation are more likely to use controlling techniques is because they more easily hinge their own self-worth upon their children's achievements. Thus, control oriented parents extend their ego-involved function to their offspring, thereby measuring their own success and worth versus failure and shame as a parents through their children's accomplishments (Wuyts, Vansteenkiste, Soenens, \& Assor, in press). Further, Soenens, Vansteenkiste, Duriez, and Goossens (2006) hypothesized that parents who have high separation anxiety may perceive expressions of their children's autonomy as threatening, and may be more likely to restrain autonomous behavior through controlling techniques. In addition, they argued that self-critical perfectionism in parents would lead them to project higher demands 
on their children, thereby pressuring toward specified outcomes. Their results supported these hypotheses.

Together such findings underscore that parenting behaviors are themselves influenced by a variety of factors both external and internal-from the socioeconomic pressures on parents that stretch their resources and lead to insecurities, to internalized social pressures to make their children achieve. Pressures on parents in the form of threats thus interrupt organismic trust, and capacities for nurturance. On the other hand, it is important to highlight that most parents, because of their inherent attachment and love for their children, naturally seek to provide support and guidance so that even their controlling behaviors are often, despite their negative consequences, expressions of their parental caring (e.g., see Chua, Wong, \& Koestner, 2014).

\section{Summary}

SDT is primarily focused on nurturing, facilitating environments that support the growth of the self. The theory specifies three operationally distinctive nutritive dimensions, namely, autonomy support, structure, and involvement. These parenting dimensions are systematically related to the satisfaction of children's basic psychological needs and allow for greater specification of optimal parenting relative to mixed constructs such as Baumrind's $(1996,2012)$ construct of authoritative parenting and confrontive power (see Grolnick, 2002, 2012). Further, in discussing the need-thwarting role of parents, we especially emphasized the role of controlling parenting, with conditional regard being the most studied controlling practice within SDT. Finally we suggested that parent behaviors do not occur in a vacuum. Parental stress, sense of threat, and ego involvement can all lead to controlling behaviors and can detract from positive involvement and capacities for autonomy support.

\section{AUTONOMY AND AUTONOMY SUPPORT IN MAJOR DEVELOPMENTAL PROCESSES: ATTACHMENT, INTRINSIC MOTIVATION, INTERNALIZATION, EMOTION REGULATION, AND IDENTITY FORMATION}

Having elucidated the critical parenting dimensions fostering growth, in this section we elaborate the role of autonomy in development and personality functioning. First, we focus on the relations between autonomy and attachment security (Bowlby, 1969). SDT suggests that autonomy support is an underemphasized yet critical predictor of secure attachment experiences (e.g., Whipple et al., 2009).
Even more, we argue that security provision is insufficient in accounting for healthy self-development, which requires the more active notion of autonomy support. We then turn to a review of research on four critical developmental processes in which autonomy figures heavily, namely, intrinsic motivation, internalization, emotion regulation, and identity formation, and we explicate the role of autonomy and the impact of contextual supports for it.

\section{Autonomy and Attachment Security}

Connections between autonomy and relatedness have been hotly debated in both developmental and social psychologies. Some authors see autonomy and relatedness as largely orthogonal if not antithetical constructs (e.g., Blos, 1979; Ingeyar \& DeVoe, 2003; Jordan, 1992). As noted already, SDT differs, viewing autonomy and relatedness as being interrelated and, indeed, integral to one another. This is so from the earliest phases of life, in which the strength and security of attachment is, according to SDT, in very large part a function of the autonomy support afforded by caregivers.

\section{Attachment and Autonomy in Infancy}

It is particularly difficult to think about autonomy without its foundations within relatedness during the child's first years of life and vice versa. During early development, establishing a core self is a primary agenda for the infant (Stern, 1985). Although the self that is consolidated within the first six months does not take the form of a conscious representation, it can manifest as coherence and volition. Yet because infants are highly dependent on caregiversnot only for biological necessities such as food and temperature regulation but also for interpersonal necessities such as love, contact, and comfort-these earliest experiences of the self as initiator of action typically occur within the context of close parent-child relationships.

Bowlby (1969) and later theorists elaborating his attachment theory framework (e.g., Mikulincer \& Shaver, 2007) have argued that early social relations with primary caregivers shape both the security of attachment and the corresponding working models of self and others that will organize future social relations. Perhaps the most emphasized feature within attachment theory that contributes to security of attachment is caregiver responsiveness or sensitivity (Ainsworth, Blehar, Waters, \& Wall, 1978). Sensitivity is defined as the provision of contingent, appropriate, and consistent responses to the child's signals and needs (Lamb \& Easterbrooks, 1981). Sensitivity thus conveys that caregivers are responsive to the child's initiations 
in ways that are empathic and appropriate to the child's needs. In our view, sensitivity is part of autonomy support, though by no means encompasses that construct.

Within SDT it has been suggested that when one unpacks the kind of parenting that fosters growth and security, chief among them will be autonomy support (e.g., Ryan, 1993; Ryan, Brown \& Creswell, 2007). As noted, in autonomy support what one is being sensitive to are the child's initiations, strivings, and the needs of the developing self (rather than the caregiver's agendas or projected needs). Indeed, Bretherton's (1987) description of sensitivity as "maternal respect for the child's autonomy" (p. 1075) captures the essence of such active responsiveness. The sensitive caretaker responds to the initiations, cues, concerns, and needs that emerge from the child, and this contingent responsiveness gradually strengthens the child's inner sense of agency and coherence, and thus the meaningfulness of the child's self-initiated action and expression. When responded to, the child experiences a sense of safety and interconnection that is lacking for one whose biddings and expressions have been neglected.

Autonomy support is thus a critical component of early parent-child relationships, for it facilitates not only the solidity of attachment but also self-development more generally. Numerous past studies support this perspective by linking sensitivity or autonomy support to more curiosity, effectance, initiative, and adjustment (e.g., Stevenson \& Lamb, 1981; Waters, Wippman, \& Sroufe, 1979; Yarrow, Rubenstein, \& Pederson, 1975). Specifically applying SDT, Grolnick, Frodi, and Bridges (1984) found that mothers rated as autonomy supportive had 1-year-old infants who both explored more and were more persistent at a play task than infants of mothers rated as controlling. Further, Frodi, Bridges, and Grolnick (1985) reported similar results for the persistence and competence at 20 month of age. Such studies suggest that in early stages of development, parental attunement and support for autonomy are enhancing the child's inner resources for self-regulation.

We suggest, in fact, that autonomy support is an aspect of caregiving that is far too underemphasized in attachment theory's foundational focus on security, protection, and responsiveness. Underscoring this argument, Whipple, Benier, and Mageau (2011) drew from SDT in using observational assessments of maternal autonomy support during infant ( 15 months) exploration. They also assessed maternal sensitivity with a 90 -item measure of the quality of maternal behaviors during a home visit and security of attachment using an observational Q-sort method. Results showed that not only sensitivity but also autonomy support explained independent variance in attachment security.
This shows how autonomy support is not encompassed by traditional conceptions of sensitivity.

This interrelation between autonomy support, attachment, and self-development is nonetheless consistent with some of Bowlby's (1969) speculations concerning infant exploration and mastery motivation. Bowlby postulated that to the extent that a child experiences security (i.e. caregiver accessibility and responsiveness in times of need), then the child will feel enabled to venture forth to explore with interest. Yet SDT would suggest, beyond Bowlby, that security is much more than protection and comfort; it is in fact responsiveness to the self of the child that fosters secure relationships, not simply reliable protection (Ryan, Brown, \& Crewell, 2007).

It is worth noting in this regard that for Bowlby, attachment was first and foremost about safety and protection rather than facilitating growth and development. As Kobak, Cassidy, and Zir (2004) stated, attachment is a "behavioral system activated by appraisals of danger and accompanying signals of fear" (p. 388). Adult attachment theorists concur, arguing that "the goal of the system is a sense of protection and security" (Mikulincer \& Shaver, 2007 , p. 5). Without doubting the importance of protection and the value of reducing anxiety in moments of danger one can question whether this is a sufficient foundation for a full theory of self-development (see Ryan et al. 2007). Although moments of dangers and strangers can be salient, they are episodic, and comfort following them may be a necessary, but not a sufficient, condition for the interpersonal support for self-development. Even among the object-relations theorists in Bowlby's time, figures such Winnicott (1965) more strongly pointed to the importance of ongoing positive supports for the nascent self to not only feel safe, but to develop and flourish. Winnicott highlighted not only a safe haven but also active empathy and having spontaneous initiations amplified through mirroring and responsive care. Like Winnicott, SDT argues that support and nurturing relationships in infancy extend well beyond security and protection to the active facilitation of self-functioning. Indeed, security and protection are likely key to prevent experiencing of need frustration, yet, for children to experience autonomy, competence, and relatedness, more active support of these needs is required. In fact, as we turn to other developmental processes such as intrinsic motivation, internalization, emotion regulation, and identity formation, the role of caregivers' support for the child's psychological needs, including autonomy, emerges as a critical facilitator of self-development.

This brief analysis of the role of autonomy and relatedness in infancy thus suggests that autonomy support is 
a critical component of caregiving in infancy that shapes both the experienced quality of relatedness (as reflected in the security of attachment) and the consolidation and vitality of the self (as reflected in both well-being and mastery motivation). Caregiving that is low in autonomy support, by contrast, will set the stage for attachment disturbances and impoverished agency.

More recent studies have begun to examine the independent role of parental autonomy support and attachment security per se in young children's development. For instance, Verschueren, Doumen, Vervoort, and Vansteenkiste (2013) showed in a longitudinal sample of preschool children that both a secure attachment and maternal autonomy support, as observed during a problem solving task, related to higher achievement on a standardized language and mathematics test taken at the end of the first elementary school year, through partially distinct pathways. Attachment security in preschool was related to greater closeness in the teacher-child relation in the first school year, whereas maternal autonomy support related to both greater teacher-child closeness and more learning based in interest and enjoyment (i.e., intrinsic motivation), which in turn predicted greater actual achievement. These findings suggest that a secure and autonomy-supportive child-parent relationship promotes a more successful transition from preschool to elementary school, with need-supportive interactions generalizing to new socialization figures.

\section{Attachment and Autonomy in Adolescence and Beyond}

There is increasing evidence that at whatever age attachment security is assessed, the provision of autonomy support from attachment figures will be a critical predictor of attachment security. Adolescence, for example, is a critical period of life in which teens are rapidly developing capacities for independence, while still needing considerable support and nurturance. As noted previously, in SDT's view, rather than individuating from parents, healthy adolescent development is characterized by continued attachment and dependence along with autonomy. In fact, evidence suggests that security of attachment and reliance on parents in this age is quite important for well-being and that these are facilitated by caregivers' support for autonomy.

First, several studies support the view that adolescents' continue to benefit from secure attachments to parents rather than by the detachment that Blos (1979) advocated. Ryan, Stiller, and Lynch (1994) investigated the contributions of felt security with parents, teachers, and friends to the motivation, adjustment, and self-esteem of early adolescents. They found that felt security with parents and teachers, as well as utilization (reliance on) these figures, predicted adjustment. Further, they found evidence that felt security with parents predicted security with both teachers and peers, and appeared to have priority among working models. Similarly, Bober and Grolnick (1995) assessed adolescents' felt security with and emulation of parents. Adolescents who displayed internalizing symptomatology reported low levels of felt security with parents yet emulated them. Adolescents with externalizing symptomatology reported low levels of felt security and low emulation of parents.

Ryan and Kuczkowski (1994) examined felt security with parents as it relates to the emergence of private audiences in the cognitive development of adolescents. Following Elkind (1967), they argued that the private-audience phenomenon initially represents a heteronomous influence on behavior for most adolescents, who become more self-conscious and more prone to conformity. Yet with self-development the salience of private audiences has been hypothesized to lessen. Ryan and Kuczkowski (1994) predicted and found that whereas felt security with parents was unrelated to the strength of private audiences in early adolescence (when it is a normative aspect of development), it was more strongly related in later adolescents. Adolescents experiencing insecurity with parents remained more preoccupied with private audiences, showing one pathway through which the growth of autonomy is hampered.

Ryan and Lynch (1989) examined the issues of autonomy and attachment in early, middle, and late adolescent samples. They argued that autonomy is typically facilitated by secure attachment to, and dependence on, parents rather than by detachment and nonreliance on them. They further suggested that among the primary reasons that adolescents are insecure with or detached from parents is that parents are overly controlling. Their studies confirmed that adolescents with more secure attachment to parents were, in fact, better adjusted, and had parents who were more autonomy supportive. These findings have been well replicated, including recent studies of both referred and nonreferred youth and their mothers, in which parental autonomy support related to lower anxiety-based attachment, while parental responsiveness and sensitivity related to lower avoidance-based attachment (Brenning, Soenens, Braet, \& Bal, 2012; Karavasilis, Doyle, \& Markiewicz, 2003).

The importance of autonomy supports for secure attachments extends to early adulthood. La Guardia, Ryan, Couchman, and Deci (2000) studied the attachment experiences of college students with multiple social partners such 
as their mothers, fathers, best friends, and romantic partners. They found, as classical attachment theory predicts, that individual differences in security of attachment were a moderately significant predictor of patterns of security of attachment across social partners. Yet they also hypothesized and found significant variability in students' security of attachment with these different social partners as a function of the different qualities of interpersonal supports provided within each relationship. In three studies, La Guardia and colleagues applied multilevel modeling to reveal that within-person variability in attachment security was systematically explained by relationship-specific autonomy support. In fact autonomy support was an especially consistent predictor of felt security, relationship satisfaction, and willingness to rely on that person.

In a more recent study of adult attachments, autonomy support, and emotional reliance, Lynch (2013) collected daily data on autonomy support and security of attachment to predict patterns of emotional reliance - that is, the individual's willingness to turn to another person for emotional support (see Ryan et al., 2005). It has been assumed by attachment theorists that secure attachments would be associated with positive abilities to recruit support from others when distressed (e.g., Belsky, 2002). Yet Ryan et al (2005) argued that autonomy support was also particularly crucial in facilitating such emotional reliance, as people are less prone to rely on others who are controlling. Lynch (2013) therefore allowed measures of both attachment security and autonomy to compete at both trait and state levels in predicting emotional reliance. At the trait level both security of attachment and trait autonomy predicted greater tendencies to emotionally rely on others. At the within-person level, results showed, as predicted by SDT, that people were specifically more willing to turn to those people whom they perceived to be autonomy supportive. Noteworthy is that at both within- and between-person levels of analysis, emotional reliance was more strongly linked with autonomy than with attachment security.

\section{Summary}

SDT and attachment theory thus concur that attachment security is associated with higher well-being and ability to rely on others. SDT additionally maintains that autonomy support is critical to experiencing attachment security, including primary attachment figures. Moreover, through the more active nurturance of explorative behaviors, emerging interests and personal values, autonomy-supportive agents do more than provide comfort; they promote children's healthy development.
Intrinsic Motivation: A Spontaneous Expression of Human Autonomy

\section{Intrinsic Motivation: Definition}

Perhaps the most direct catalyst of empirical research on autonomy within both social and developmental psychologies has been interest in the phenomenon of intrinsic motivation. Intrinsic motivation is defined as motivation derived from the inherent satisfactions of an activity - it applies when actions are experienced as interesting or enjoyable for their own sake (Deci \& Ryan, 1985b)

Harlow (1950) was, to our knowledge, the first scholar to employ the term intrinsic motivation. He used it in reference to the persistence of primates, curiously working at mechanical puzzles without reinforcements or rewards. In fact, he was an early observer of an "undermining effect" of rewards, noting that attempts to enhance curious behaviors with reinforcements disrupted such intrinsic motivation Subsequently, White (1959), drawing on both comparative psychology and developmental studies, argued that this type of interest-driven behavior underlies much of human social and cognitive development. Indeed, this idea of an innate, natural propensity towards the exercise and growth of capacities has been an important postulate in the study of developmental and learning processes (Deci \& Ryan, 2013; Flavell, Miller, \& Miller, 2002; Koestner \& McClelland, 1990).

Intrinsic motivation reflects a natural assimilative tendency, which is evident in the active exploration, manipulation, curiosity, and playful exercise of skills we observe in healthy children. Intrinsically motivated play and activity foster learning and the growth of competencies, as developmental theorists have long argued (Harter, 2012). Also studied as mastery motivation, intrinsic motivation is clearly evident in infancy (e.g., Yarrow et al., 1975) and differentiates into more specific interests as children grow (Deci \& Ryan, 2013). Numerous studies have linked intrinsic motivation to deeper learning (e.g., Grolnick \& Ryan, 1987; Yamauchi \& Tanaka, 1997), creativity (e.g., Amabile, 1983), and other indicators of high-quality assimilation.

\section{Social Contexts and Intrinsic Motivation}

Although intrinsic motivation is posited to be natural, its unfolding and development is nonetheless viewed within SDT as being dependent on specific social and contextual nutrients (Deci \& Ryan, 1985b; Ryan, 1993). A substantial amount of research has therefore explored the effects of various social contexts on intrinsic motivation, and this 
body of research has been described within a specific mini-theory of SDT called cognitive evaluation theory (CET; Deci \& Ryan, 1985b). In brief, CET argues that intrinsic motivation is dependent on experiences of both autonomy and competence. Social contexts that are either controlling or autonomy inhibiting (Grolnick \& Ryan, 1989) or that lack challenges or feedback that supports felt competence (e.g., Abuhamdeh \& Csikszentmihalyi, 2012; Mouratids et al., 2013) are expected within CET to diminish intrinsic motivation. In contrast, environments that support autonomy (e.g., provide choice, are responsive to initiatives) and competence (are well structured, provide positive feedback) are expected to maintain or enhance intrinsic motivation. Finally, in contradistinction to social-cognitive approaches (e.g., Bandura, 1989), CET holds that that intrinsic motivation requires autonomy satisfactions; self-efficacy alone will not sustain this spontaneous form of activity.

Studies testing CET have been done with toddlers to adults, allowing us to draw inferences about some fundamental, age-invariant aspects of intrinsic motivation. One type of evidence comes from experimental studies. In the typical paradigm children or adults work on an activity under different conditions that vary in terms of contextual factors affecting perceived autonomy, perceived competence, or both. Subsequently, intrinsic motivation is assessed by a free-choice behavioral measure. Participants are given a postexperimental period without demands, in which the target activity is available along with alternative activities (e.g., reading a magazine). The time participants spend spontaneously engaging the target activity is recorded and used as the free-choice measure of intrinsic motivation. Ratings of how interesting and enjoyable participants find the task are a complementary measure of intrinsic motivation.

One interesting factor affecting intrinsic motivation is external rewards. CET postulates that because rewarded participants are more likely to see the impetus or cause of their activity as stemming from external sources, when they are offered rewards for acting they are vulnerable to losing an internal perceived locus of causality and sense of autonomy. When originally proposed (Deci, 1971), this CET hypothesis was provocative because many psychologists then held a view, based in operant psychology (Skinner, 1953), that all behavior was controlled by external contingencies of reward and punishment. CET not only assumes this is not the case, but moreover suggests that some types of rewards that are very effective in shaping behavior can at the same time yield detrimental effects on intrinsic motivation, precisely because of their negative effect on autonomy. In contrast when rewards are not controlling, but rather provide more informational (or competence relevant) feedback, they can be more motivationally positive (Ryan, Mims, \& Koestner, 1983).

Danner and Lonky (1981), combining Piagetian and CET frameworks, presented a classic study illustrating some of the developmental implications of this undermining effect of rewards on intrinsic motivation. Children were classified with regard to their level of cognitive development using a Piagetian assessment. They then worked on additional cognitive tasks either explicitly for, or without, externally offered rewards. Subsequently, children were given a free choice period in which they were allowed to select activities during a play opportunity. Children who did not receive external rewards gravitated to tasks that were optimally challenging - those stretching their assessed cognitive capacities. In contrast, children who had been externally rewarded sought easier tasks and avoided challenges during free play. These results illustrate how attempts to enhance development through controlling rewards can backfire

Another example comes from studies of young children's intrinsic interest in helping others, which many argue is a deeply evolved human propensity (see Ryan \& Hawley, in press). To demonstrate that early altruism constitutes an intrinsic or a natural inclination Warneken and Tomasello (2008) created situations where 20-month-old toddlers could help adults (e.g., pick up dropped objects). In one condition they gave children a concrete reward every time they helped, whereas children in another condition did not get rewards. In a second phase, they found that children previously rewarded for helping were less helpful than those never rewarded. Presumably, the reward undermined children's spontaneous, intrinsically motivated tendency to be helpful, a finding paralleling adult findings (e.g., Weinstein \& Ryan, 2010). This experiment is notable in finding the effect in children before they can cognitively weight or discount the motives underlying their behavior, ruling out so-called overjustification explanations of this effect (e.g., Lepper, Greene, \& Nisbett, 1973).

Despite a plethora of studies documenting undermining effects, debate about the undermining effect was resparked by Eisenberger and Cameron (1996). who presented a meta-analyses of reward effects that seemingly contradicted previously published summaries and meta-analyses on the topic. In their analysis they surprisingly found no evidence for an undermining effect by rewards, and they labeled the undermining effect a myth. Their null findings were immediately heralded by many behaviorists. Yet detecting serious methodological problems, Deci, Koestner, and 
Ryan (1999) reanalyzed these experimental data, taking care to include all studies summarized by Eisenberger and Cameron (1996). The reanalysis, presented in Psychological Bulletin, not only confirmed this well-known undermining of intrinsic motivation by rewards, but more importantly, supported the differentiated pattern of reward and feedback effects on intrinsic motivation predicted by CET. Data indicated that controlling reward contingencies undermined intrinsic motivation, whereas noncontrolling and informational rewards did not.

Many studies continue to document this important effect. One study of particular interest focused on neurological supports for intrinsic motivation (Murayama, Matsumoto, Izuma, \& Matsumoto, 2010), providing a new way of demonstrating the undermining effect of rewards on intrinsic motivation. These researchers had participants engage in an interesting task under reward and no-reward conditions, tracking activations with fMRI. The no-reward group found the task interesting and cognitively engaging, and these inherent satisfactions were associated with meaningful striatal and lateral prefrontal cortical activity. For the reward group, Murayama et al. (2010) provided a performance-contingent monetary reward, and as predicted both striatal and lateral prefrontal cortical activity increased significantly, suggesting that the reward added to task motivation and engagement. Yet in a second phase of the study, participants repeated the task, but in this phase no rewards were offered. The researchers again examined the striatal and lateral prefrontal cortical activity the task generated. For participants in the prior no-reward condition, striatal and lateral prefrontal cortical activation were essentially stable; the task remained just as rewarding and engaging as before. Yet for those in the reward condition, striatal and lateral prefrontal cortical activity declined - the task now failed to generate pleasure (striatum) or cognitive engagement (lateral prefrontal cortical), as predicted by CET.

SDT scholars do not question the power of rewards to control behavior. However, SDT emphasizes that, at the same time, the very power of externally administered rewards can also lead individuals away from their intrinsic inclinations. Powerful extrinsic rewards, whether they be tangible rewards or conditional love, can lead people to forego autonomy, forget their values and preferences, and neglect their interests and relationships. That is precisely what unhealthy regulation and alienation is all about (Ryan \& Deci, 2000a). Not only can controlling rewards undermine intrinsic motivation, so can motivational tactics such as controlling praise (e.g., Ryan, 1982); threats of punishment (e.g., Deci \& Cascio, 1972); salient surveillance (e.g., Enzle \& Anderson, 1993; Plant \& Ryan, 1985); controlling-directive language (e.g., Hooyman, Wulf, \& Lewthwaite, 2014; Reeve \& Jang, 2006); and grades (e.g., Pulfrey, Buch, \& Butera, 2012). These and other frequently used methods of controlling behavior can have a psychological meaning, or functional significance, of being controlling, which in turn negatively affects people's experience of autonomy and engagement. To the extent that adults use salient prods, prompts, or pressures to induce children to perform interesting activities, they are likely to disrupt intrinsic motivation and autonomous functioning (Assor et al., 2004).

\section{Effects Across Developmental Period}

Although many experimental studies of intrinsic motivation have been in school and college settings, the effects of autonomy support versus control on intrinsic motivation are robust across developmental epochs and interpersonal settings. We review some examples.

Earlier we cited studies by Grolnick and colleagues $(1984 ; 1985)$ in which infants and toddlers whose mothers were more controlling evidenced less mastery motivation and persistence than those whose mothers more actively supported their initiations and autonomous play As another illustration, Grolnick et al. (2002) observed mothers and their third-grade children working together on an assigned task of problem solving and constructing poems. Following the interaction, children were asked to complete similar tasks on their own, without their mothers being present. Children whose mothers interacted with them in a more controlling manner had performed just as well as other children when with their mothers, but, when completing tasks on their own, they were less accurate in problem solving, and wrote less creative poems.

Deci, Driver, Hotchkiss, Robbins, and Wilson (1993) looked at maternal autonomy support and control with five- to seven-year-olds. They recorded mothers' vocalizations during an interactive-play period, and had raters code the interactions for autonomy supportive and controlling contents. After sessions in which mothers were rated, children's intrinsic motivation was assessed using persistence observations during solo play. Children whose mothers were rated as more autonomy supportive were more intrinsically motivated.

The significance for development of this general effect concerning autonomy support and control on intrinsic motivation cannot be overstated. Children's exploration, challenge seeking, and curiosity are inherent and natural resources for cognitive and emotional development, and a wellspring from which specific competencies derive. 
Also, when led by curiosity and exploration, children gradually learn to discover their interests, a first step toward pursuing their interests and gradually building their skills. Yet this resource is curtailed by controlling environments. Intrinsic motivational tendencies that support growth can be stifled by even well meaning caretakers as they attempt to elicit development through reinforcement or control.

The significance of intrinsic motivation, and the impact of social contexts on it, has been documented in various domains, including education, exercise, and work. For example, within schools Deci, Schwartz, Sheinman, and Ryan (1981) collected teacher's reports about their classroom motivational strategies before they met their students for the year. Several weeks into the school year Deci et al. (1981) returned to assess student motivation and well-being. They found that students assigned to teachers who were oriented toward supporting autonomy displayed higher levels of curiosity, perceived cognitive competence, and self-esteem than those assigned to teachers espousing more controlling orientations. Ryan and Grolnick (1986) reported parallel results using elementary school children's perceptions of their teachers. Notably, even in an "elective" domain such as sport, O'Rorke, Smith, Small, and Cumming (2012) found that young athletes' autonomy for playing sport was related to the motivational climate created by parents. Parents focused on mastery motivation with little ego-involvement had children with higher levels of autonomy and intrinsic motivation for sport. A prospective analysis over the course of a sport season showed, in fact, that ego-involved parents had children whose autonomy for playing decreased over the season.

Indeed there is now an extensive literature that continues to document the beneficial effects of autonomy-supportive socialization, including their generalizability across cultures. For example, Chirkov and Ryan (2001), studied Russian and U.S. high school students, finding that perceived autonomy support from parents and teachers was associated with more internalized academic motivation and better mental health in both samples, to comparable degrees. Jang, Reeve, Ryan, and Kim (2009), in a prospective study of South Korean high school students found that teacher autonomy support predicted greater intrinsic motivation in school. Jang, Kim, and Reeve (2012) extended these findings in a multiwave longitudinal study of more than 500 South Korean students, testing a complex path model. As expected, teacher autonomy support enhanced student need satisfaction and engagement, resulting in better achievement. But reciprocal effects were also apparent, such that when students' need satisfaction was high, teachers were influenced to be less controlling. This kind of longitudinal approach reveals the complex dynamic effects between autonomy support and autonomous motivation, which are truly interpersonal and in which influences can be reciprocal.

Intrinsic motivation is quite contextually sensitive, fluctuating over time with perceived autonomy support. For example, Tsai, Kunter, Ludtke, Trautwein, and Ryan (2008) showed that German public school students, followed over multiple days in three different required courses, showed considerable lesson-to-lesson variability in interest. Yet the fluctuations were systematic: they were directly associated with variability in teacher autonomy support. On days when the teacher was more controlling students' interest waned. These findings were extended by Mouratidis, Vansteenkiste, Lens, and Sideridis (2011). Rather than following daily variations in perceived teaching behavior, they instructed teachers to randomly build in autonomy supportive segments within their more typical teaching approaches. In classes where the strategies were inserted adolescents reported enjoying the class more and feeling more vital and energized. Moreover, these effects from inserting autonomy-supportive strategies were specifically accounted for by students' reports of autonomy need satisfaction.

Factors that support a person's autonomy can, of course, enhance interest and intrinsic motivation. For example, a meta-analysis by Patall, Cooper, and Robinson (2008) showed that in general the affordance of meaningful choices to people has an enhancing effect on intrinsic motivation (see also Katz \& Assor, 2007). Autonomy support is also conveyed by taking the person's internal frame of reference, or understanding their point of view (Deci \& Ryan, 1985b; Koestner, et al., 1984). In fact, Patall, Dent, Oyer, and Wynn (2012) found that teachers' perspective taking and provision of choice were both autonomy-enhancing factors for high school students. Such studies indicate that providing choice and acknowledging perspectives can enhance intrinsic motivation, as these events leave individuals feeling more autonomous.

\section{Summary}

Experiments and field studies in diverse domains, including parenting, teaching, and coaching suggest that social contexts that are experienced as controlling (i.e., as pressure to think, feel, or behave in specific ways) undermine autonomy, resulting in diminished intrinsic motivation and more negative self-related emotions. In contrast, contexts that are autonomy supportive (i.e., that consider 
the individual's frame of reference, provide choice, and encourage self-initiation) have positive effects on intrinsic motivation and persistence.

Internalization: Assimilating Social Regulations and Values

\section{The Internalization Continuum}

Intrinsic motivation is a prototype of autonomous motivation that occurs spontaneously, as an expression of our active, assimilative human nature. Many behaviors, however are not intrinsically interesting, but parents, school systems, or other authorities believe them to be in children's best interests and therefore important for them to acquire. Although parents and teachers, as primary socializing agents, attempt to motivate children to perform such activities, the methods they use vary. Some may be controlling and authoritarian, forcing the child to conform to norms or expectations; others may be autonomy supportive, influencing through reasoning, modeling, and guidance of choices. SDT predicts that whereas controlling methods foster at best short-term compliance, autonomy support structure, and involvement in conjunction enhance the internalization and integration of behavioral regulations.

Ryan, Connell, and Deci (1985) defined internalization within SDT as the means through which individuals can become more autonomous in performing an activity that was initially externally prompted. In this view internalization is an active process of selecting and transforming ambient regulations into personally endorsed values or motivational propensities. Through internalization, people acquire values and behaviors that allow them to be effective, connect with others, and experience volition - that is, to get their psychological needs met. When functioning optimally, the internalization process results in values and regulations that are more self-endorsed, that is, are fully integrated within the self

SDT further hypothesizes that supports for autonomy, competence, and relatedness are necessary for such optimal, integrated internalization (Deci \& Ryan, 2000; Ryan, 1993). Unfortunately, these ideal contextual conditions are not widely prevalent, and full integration of regulations is not often attained. Instead, when socializing agents are controlling or uninvolved, internalization results in values and regulations that are only poorly internalized. SDT therefore places importance on distinguishing between types of internalization for predicting the nature and quality of behavioral and psychological functioning. Specifically, SDT specifies a continuum of regulatory styles that spans from low to high autonomy for acting.
At the low end is external regulation, which describes extrinsically motivated behavior that is initiated and maintained by external rewards or punishment avoidance. A child who does chores to avoid parental nagging would be externally regulated, as would one who does them to get tangibly rewarded. External regulation is characterized by a lack of internalization, and although external rewards and punishments can be powerful motivators, externally regulated behavior is poorly maintained in the absence of direct control.

Somewhat more autonomous is regulation based on introjection. Introjected regulation is motivated by feelings of self-worth and loveability for acting as one should, and guilt or self-disparagement for failing to meet the standards or goals. Introjected regulation is thus often rigid and accompanied by feelings of internal pressure and tension. A child who does chores because she feels pressured to avoid feeling guilty and to feel like a "good girl" is displaying introjection. Indeed, introjection supplies the developmental basis for internally controlling regulation, as exemplified in ego involvement (Ryan, 1982), contingent self-worth (Deci \& Ryan, 1995; Kernis, 2003), and self-critical perfectionism (Frost et al., 1993; Soenens, Vansteenkiste, Duriez, Luyten, \& Goossens, 2005). Accordingly, research has related introjection to rigidity, anxiety, self-derogation, and other maladaptive patterns (e.g., Assor, Vansteenkiste, \& Kaplan, 2009; Weinstein, Deci, \& Ryan, 2011).

Still somewhat higher on this continuum of autonomy is identified regulation of behavior. In this type of regulation the person consciously identifies with a value or behavioral goal-accepting it as his or her own. It is therefore enacted with less resistance and conflict, and often with persistence, diligence, and efforts based in valuing. Children who clean their rooms because they understand that a clean room allows them to more easily find what they need have concurred with the importance of cleaning, that is, they have more fully internalized the value and reason for cleaning. Finally, integrated regulation represents the most autonomous form of internalized regulation, as it indicates that an identified value and its accompanying regulatory process have been reciprocally assimilated with other values and important personal goals. Various identified values may be more or less challenging to integrate, but if integrated, are wholeheartedly endorsed and performed

These four modes of extrinsic regulation lie along a continuum of relative internalization and autonomy, where external regulation represents the least internalized and autonomous mode and integrated regulation represents the most internalized and autonomous mode of extrinsically 
motivated behavior. Intrinsic motivation is also on the high autonomy end of this dimension, yet does not require internalization of regulation as the behavior already spontaneously manifests when people act out of enjoyment and interest. Considerable evidence backs up the continuum nature of this array. Ryan and Connell (1989) provided the initial studies assessing children's external, introjected, identified and intrinsic regulatory styles, and their data confirmed that these were reliably ordered along a continuum of increasing autonomy. Moreover, the more autonomous forms of regulation (identified and intrinsic) were positively correlated with positive affect and proactive coping, whereas the less autonomous forms (external and introjected) were correlated with negative affect and maladaptive coping. Introjection, in particular, was associated with anxiety and anxiety amplification following failure, highlighting the inner stress and tension associated with this internally controlling form of regulation.

Enhancing internalization and integration is a valuable goal in its own right, yet when students have more autonomous forms of motivation they also tend to more deeply process the learning material (Sobral 2004; Yamauchi \& Tanaka, 1998), to be more fully absorbed in the task at hand (e.g., Abuhamdeh \& Csikszentmihalyi, 2012), and to perform better. For example, De Naeghel, Van Keer, Vansteenkiste, and Rosseel (2012) examined elementary students' motives for reading, both in school and in leisure time. They found that more internalized, autonomous motives not only related positively to teacherrated engagement in reading, but also to children's scores on a standardized reading comprehension test. At the other end of educational development, Kusurkar' TenCate, Vos' Westers, and Croiset (2013) found that autonomous motivation is positively associated with the use of a deeper study strategy and better GPA in medical students. In fact, a recent meta-analysis by Cerasoli, Nicklin, and Ford (2014) supports this pattern of findings, with intrinsic/ autonomous motivation predicting both better quality and quantity performance, while incentives related only to better quantity performance.

The relations of internalization and autonomy to higher quality behavior are not limited to learning and achievement domains. For example, Ryan and Connell (1989) found that more autonomous regulation in the prosocial domain was associated with greater empathy, more mature moral reasoning, and more relatedness. Blais, Sabourin, Boucher, and Vallerand (1990) assessed adults' reasons for maintaining their primary relationship, finding that regulatory styles representing lower autonomy were negatively associated with dyadic adjustment and relationship satisfaction. Ryan, Rigby, and King (1993) examined the internalization of religious values, finding that introjected regulation of religious behaviors was positively associated with symptoms of depression, anxiety, and low self-esteem, whereas identified regulation was negatively associated with these indicators of ill-being. Neyrinck, Vansteenkiste, Lens, Hutsebaurt, and Duriez (2006) found that greater internalization of religious activities was associated with a more open and flexible interpretation of doctrines, stronger adherence to beliefs, and greater well-being. In the health care domain, Williams, Rodin, Ryan, Grolnick, and Deci (1998) showed that patients who had more strongly internalized the self-importance of taking their medication were more likely to effectively take them. Finally, although parents constitute primary socialization figures in the transmission of values, only the autonomous agreement with parental values relates positively to well-being, even after for controlling for the degree of parent-child agreement as such (Knafo \& Assor, 2007). Across these domains and others, findings consistently show that the more autonomous people's motives, the more effective their functioning and psychological experiences.

\section{Social Contexts and Internalization}

Given these benefits of fuller internalization, the developmental conditions that foster or facilitate internalization and autonomous regulation are an important focus of research. Although like intrinsic motivation, internalization is hypothesized within SDT to be a natural process (Ryan \& Hawley, in press), it too requires specific nutrients and supports from the social context. In fact, all three needs are critical to foster the full integration of socially prescribed norms and values. That is, a sense of competence is an important yet not sufficient condition for fuller internalization of an activity, as one can feel fairly competent, yet externally pressured to do the activity. Similarly, a sense of relatedness is conducive to taking in the behavioral regulation, yet a sense of internal conflict can still be underlying the behavior if it is emitted to avoid feelings of disloyalty and guilt vis-à-vis the socialization figure. For full internalization to occur, a sense of autonomy is critical. Consistent with this idea, Markland and Tobin (2010) showed that the satisfaction of the three needs maps in theoretically meaningful ways to the different types of regulation for exercising. Whereas relatedness satisfaction yielded a unique positive association with introjected regulation, it is only when all three needs were fulfilled, that the exercisers identified with the importance of exercising.

To promote autonomy need satisfaction and subsequent internalization, the use of control has been found 
to be harmful, whereas provision of autonomy support has been shown to be critical, a finding that generalizes across cultures (e.g., Ahmad, Vansteenkiste, \& Soenens, 2013; Barber, Stolz, \& Olsen, 2005; Chirkov et al., 2011; Jang et al., 2009). There are many elements to autonomy support, but they all start from an empathic grasping of an individual's perspective so as to better understand why the person refuses to engage in the noninteresting activity. By taking the frame of reference of the that person, the socializing agents can provide a more relevant and meaningful rationale, provide additional competence-enhancing information, if needed, and offer choices concerning how and when an activity is carried out. Along with support for relatedness, provision of such autonomy support and structure deepens internalization and contributes to social integration and wellness.

The critical role of need-supportive socialization for children's internalization has been documented in dozens of studies. For instance, in the study by Grolnick and Ryan (1989), parents who were rated as more autonomy supportive from interviews had children whose motivation for schoolwork was more fully internalized. Notably, many parents who were rated as controlling were clearly involved in their children's lives, and many were well intended, some highly invested in their children's success (Wuyts et al., in press). Yet in attempting to control the children to ensure that they attained certain outcomes, they often failed to promote their children's ownership of these goals or the children's personal investment in achievement, instead creating a reliance on external regulation.

As noted, the practice of parental conditional regard (PCR), or the degree to which a parent shows more attention and affection after compliance or success, and less affection and attention after noncompliance or failure, has received increasing recent attention. For instance, Assor et al. (2004), studying four domains (i.e., academics, sports, prosocial behavior, emotion regulation), showed that student perceptions of PCR, assessed separately for mothers and fathers, predicted compliant behavior that was largely based in introjection and inner compulsion. PCR appeared also negatively associated with identification and feeling a sense of choice about the activities, whereas PCR was positively related to contingent self-esteem, shame, guilt after failure, and short-lived satisfaction after success - all variables one would expect to be associated with introjection. Most tellingly, students who experienced more PCR also reported more anger and resentment toward their parents

Interestingly, the negative repercussion of PCR may not only manifest at the personal, but also at the interpersonal level. Moller, Roth, Niemiec, and Deci (2014) found that late adolescents who experienced parents as being higher on PCR not only reported less secure attachments with them; they also reported more conditional regard and less secure attachments with their current romantic partners and best friends. The experience of being conditionally regarded thus appears to generalize from experiences of parents to other close relationships. Along similar lines, Soenens, Vansteenkiste, Goossens, Duriez, and Niemiec (2008) found that parental psychological control, which compromises conditional regard as one of its central components, was associated with more physical aggression and poorer peer relationships

Conditional regard, as a form of internally controlling parenting, can be differently valenced-it can take a more seductive form of positive parental conditional regard, involving the provision of more attention and affection than usual in case of desirable child behavior and a more punitive form of negative conditional regard, involving the withholding or even withdrawal of attention and affection in case of undesirable child behavior. Although most theorists might expect negative PCR to be problematic, some approaches (e.g., many social learning and cognitive approaches) might argue that positive PCR is an effective form of parenting. Indeed, some theorists appear to justify and even advocate tiger mom tactics that involve the display of PCR (e.g., Fu \& Markus, 2014). Unfortunately, it appears the also positive PCR comes with a cost. For instance, Roth, Assor, Niemiec, Ryan, and Deci (2009) showed that parents using positive PCR to promote academic success had adolescents who reported internal compulsion to do school work rather than a sense of autonomy, and their teachers' reported them to be grade-focused. In addition, extending Assor et al.'s (2004) findings, these adolescents' reported greater resentment toward parents. Negative PCR was also related to resentment of parents, and was related negatively with school engagement. Thus negative PCR does not promote internalization (not even introjection) although years earlier Sears et al. (1957) had proposed that it would. Most likely the parents who used love withdrawal in their samples also used positive conditional regard, and it may have been the positive PCR that led to their proposal. In a second study by Roth et al. (2009), positive PCR was compared to autonomy support as parenting methods for children's school achievement. Results showed that both mothers' and fathers' positive PCR were positively related to the child exhibiting grade-focused engagement, and negatively to interest-focused engagement. Further, these relations were mediated by the experience of internal compulsion 
(i.e., introjection), as predicted. In contrast, autonomy support from each parent predicted interest-focused engagement, a relation that was mediated by a sense of choice.

While internally controlling strategies, such as conditional regard, may elicit partial internalization of parental demands through introjection, parents' reliance on external controls is likely to merely elicit external regulation, representing a complete lack of internalization of the behavioral regulation. Even more, in response to external controls, children may be noncompliant or even reactive. In an illustrative observational study among Mexican children, Brunstein (1994) showed that parents' engagement in externally controlling practices (e.g., orders, reprimands, and threats) related positively to both children's observed inattentive obedience and their passive and rather blunt resistance to comply with parents' requests. More recently, Talwar and Lee (2011) conducted a field experiment comparing two West African schools, which strongly varied in terms of their use of physical punishment as a discipline practice. Specifically, 3-4-year-olds of the one school, who were very frequently exposed to the physical punishment, were 12 times more likely to lie during a toy game and to conceal their lies.

Thus, for socializing agents to promote internalization and ownership of regulations, a controlling approach would best be avoided. Yet the absence of control does not imply the absence of guidance, as would be the case with a laissez-faire climate. What is critical for children to begin to internalize societal expectations is that prescribed behaviors and limits need to be clearly communicated; in other words, sufficient structure and guidance need to be provided. Furthermore, in communicating and monitoring these limits, an autonomy-supportive style is essential.

Consistent with this, Vansteenkiste, et al. (2014) showed that the style of introducing prohibitions to adolescents affects their acceptance of (i.e., internalization) versus oppositional defiance against the prohibitions. Specifically, their one-year longitudinal study revealed that a more autonomy-supportive style of introducing and monitoring prohibitions elicited greater identification with and ownership of the limits, while a perceived controlling style predicted either increases in externally enforced compliance with the prohibitions or oppositional defiance against them. Notably, these effects were found to be reciprocal such that a virtuous positive cycle unfolded when autonomy support was salient, and a vicious negative cycle was instigated in the case of a controlling style. These effects occurred regardless of whether the prohibitions had a more personal character (e.g., friendships) or were moral in nature.

\section{Summary}

A crucial task for socializing agents is to transmit norms, values, and regulations, such that children and adolescents will not stick to these norms out of strict, slavish obedience but rather out of volitional commitment, presumably because they have come to fully own (i.e., internalize) the norms. Whereas controlling socialization practices, such as positive PCR from parents, can produce compliant behavior, it is often at the cost of a sense of volition to behave. Negative PCR and more externally controlling practices may even be more counterproductive, eliciting at best externally driven obedience, and, at worst, anger and resentment as well as blunt defiance against requested norms. What is needed to foster full internalization of norms is an autonomy-supportive approach in combination with clear communication of expectations (structure) and involved guidance.

\section{Emotion Regulation}

\section{Distinguishing Different Styles of Emotion Regulation}

Similar to the process of internalization, the development of emotional self-regulation can be conceptualized as movement from reliance on outside sources to identify, modulate, and regulate affect, toward a growing capacity for autonomous, flexible, and adaptive regulation (Cicchetti, Ganiban, \& Barnett, 1991; Grolnick et al., 1996). Deci and Ryan (1985b) early on drew from Greenspan (1979) in referring to the development of regulatory processes for autonomously managing emotions as integration at the internal boundary. This was contrasted with the development of regulatory processes for engaging in behaviors deemed important by the social world as integration at the external boundary. Although these two developmental functions, referred to, respectively, as the development of emotion regulation and internalization, are distinct, they are similar in many respects. Both involve gaining the capacities to regulate oneself with respect to behavior that is not intrinsically motivated and both entail a developmental progression in which the child gradually relies less on cues and structures from the social context and more on internal cues and structures. Equally clear, deficits in the regulation of emotion and the ability to use emotions to appropriately inform or guide behavior are cardinal features of most forms of psychopathology. Accordingly, we turn now to this critical issue of emotional regulation and its healthy development.

We begin with acknowledging the complexity of emotions, and the multiple dimensions that impact upon their regulation and expression (see Cacioppo, Bernston, 
Sheridan, \& McClintock, 2000). In particular, there are salient individual differences in people's tendencies to experience emotions (e.g., Gable, Reis, \& Elliot, 2000) based on both genetic and temperament-based factors and their interactions with early environmental events. Individual difference factors make the problem of emotional regulation and integration much more formidable for some persons, whose sensitivity or lack thereof make the task of accessing, modulating, expressing, and using emotions much more challenging (Assor et al., 2004). Nonetheless, any reasonable summary of that complex literature makes clear that even given such individual differences or tendencies, social environments play a significant and interactive role in fostering healthy emotion regulation (Vansteenkiste \& Ryan, 2013). To more concretely understand what it is about social environments that can facilitate versus debilitate a growing person's capacities for emotional awareness and regulation first requires a clear conception of what healthy emotion regulation involves.

SDT's conceptualization of healthy emotional regulation begins with the assumption that emotions are informational inputs essential to guiding action and growth (Deci \& Ryan, 1985b). In this view, specific emotions are neither good nor bad; they simply provide organismically valuable guidance (Deci \& Ryan, 2000; Parrott, 1993). A similar view is expressed by Levenson (1999), who suggested that emotions provide organisms with a built-in feedback system that signal them to inspect, explore and evaluate their environment and act in accord with that evaluation.

SDT views wellness in terms of a person being fully functioning - being able to exercise potentials, connect with others, find meaning, and experience vitality. This eudaimonic view (Ryan, Curren, \& Deci, 2013) suggests that rich access to both positive and negative feelings, a capacity to express them, and the ability to integrate and use them to inform one's behaviors and goals is optimal. This in turn supports personal growth, self-acceptance, and abilities to intimately connect with others.

To capture this quality of emotional regulation we distinguish emotional integration as an autonomous form of emotional regulation from notions of emotional control and emotional dysregulation. Specifically, emotional integration involves a differentiated awareness of one's emotional states and the capacity to use this sensitivity and awareness in the volitional regulation of action. Autonomous behavioral functioning is dependent on emotional integration, because emotions, when openly received, supply essential information that guides one in setting goals and adjusting them toward the fulfillment of psychologica needs (Ryan et. al 2006). Healthy emotional integration is thus characterized by an allowing of and interest in inner experience, rather than the control or stifling of emotions (Deci, Ryan, Schultz, \& Niemiec, in press).

Yet emotions can be experienced as more than just information; they can be experienced as evaluative and controlling, as when affect overwhelms self-regulation or automatically drives action. Even the etymology of the term emotion conveys this idea of a force that propels motion. Alternatively, emotions can be so overcontrolled such that people lose access to them, and in such cases the emotions can fail to move the individuals when they should. Controlled emotional regulation is manifest through the ignorance, suppression, or denial of emotions and, as we will see later, is implicated in specific forms of psychopathology and ill-being. Accordingly any conception of healthy or integrated emotional regulation is based neither on emotions controlling people nor on people controlling (e.g., suppressing) their emotions. When the self can access feelings without being controlled by them, the opportunity for autonomous self-regulation is maximized. This of course is a capacity that only develops over time and that requires contextual supports, in the form of guidance and need satisfaction.

Indeed, the process of increasingly integrating one's emotions and regulatory capacities is a continuation of the work a child does in gaining regulatory capacities with respect to emotions, but it represents a mature version of that process. Whereas emotional regulation is concerned with the modulation of emotional experience and expression, emotional integration is concerned with the flexibility and choice people feel in the regulation of emotions and emotion-related actions. Individuals high in emotional integration are more likely to be receptive to emotiona signals, to take interest in them, and to experience them as valuable inputs to actions. In contrast, if regulatory processes are rigidly introjected and thus in conflict with the emotions, people are likely to suppress the feelings and thus ignore their personal meaning.

Consistent with the idea that integrative emotional regulation allows for a more open and nondefensive processing of emotion-related stimuli, Roth et al. (in press) showed that, after having seen a fear-eliciting movie, individuals high in emotional integration were more likely to make use of self-referenced words, to make use of causal language and to write in the past tense, all indicative of a more open and full processing of the emotional event. In contrast, individuals high in suppressive regulation made use of fewer self-referenced and past tense wording, while at the same time using less negative emotional words, all indicative of the avoidance and lack of processing of the negative event. 
This capacity for integrative emotion regulation of one's fear and sadness has also been found to promote a greater ability to support a romantic partner's difficulties in handling negative emotions (Roth \& Assor, 2012). In contrast, suppressive regulation or dysregulation of fear and sadness was found to compromise individuals' capacity for intimacy. Individuals who tended to suppress fears and sadness reported more difficulties in disclosing negative emotions vis-à-vis their romantic partner, as well as feeling less capable of providing emotional support to their partner. Individuals who tended toward emotion dysregulation equally reported being less able to support their partner emotionally, presumably because they were so overwhelmed by their own negative emotions. Brenning, Soenens, Van Petegem, and Vansteenkiste (in press) further showed that self-esteem is affected by emotion regulation. They reported that increases in integrative regulation covaried with increases in self-esteem among young adolescents over a one-year interval. In contrast, increased use of suppressive regulation predicted increases in depressive symptoms

\section{Autonomy as an Antecedent of Emotional Integration}

A number of studies have examined whether greater autonomy would be associated with more effective emotion regulation, and better long-term coping with negative events. For instance, Weinstein and Hodgins (2009) had participants view an emotionally distressing movie twice, at least a day apart. The film was a graphic HiroshimaNagasaki documentary that was challenging to assimilate. In an initial study they showed that participants with greater trait autonomy, when given an opportunity to express emotional reactions, later had more positive well-being, energy, and memory after the second viewing. In a second study they created situational motivation by priming autonomy and control. Results showed that dispositionally controlled individuals received the same benefits as autonomous individuals, but only when primed with autonomy and encouraged to express emotions. Weinstein and Hodgins (2009) coded the contents of the emotion expressions and found that the positive outcomes associated with autonomy were mediated by nondefensive processing, as reflected in varied indices such as more self-referencing, more words reflecting internal cognitive processes, and lower use of concrete words. In short, their results suggest that autonomy facilitates ownership and expression of feelings, enhancing emotional integration over time.

Koestner, Bernieri, and Zuckerman (1992) explored the relation of autonomy to the integration and synchrony of emotions, cognitions, and behavior. They separated participants into a more autonomy-oriented group and a more control-oriented one (Deci \& Ryan, 1985a) and then explored the consistency of behaviors, attitudes, and traits within each of these two groups. In the first of two experiments, they found high correlations between behavioral persistence and self-reports of intrinsic interest within the autonomy-oriented group, but no significant correlations within the control-oriented group, suggesting less congruence in the latter. In a second study, Koestner et al. (1992) had participants complete a measure of conscientiousness, and then gave them an opportunity to behave conscientiously in succeeding days. Participants' self-reported conscientiousness and their actual conscientious behaviors were significantly more highly correlated within the autonomous group than the controlled group. Self-ratings and peer ratings of conscientiousness were also more highly correlated for the autonomy oriented. Together these studies provide support for the proposition that autonomy is associated with greater congruence among traits, behaviors, and feelings, reflecting greater integration in personality functioning.

Related to the issue of emotion regulation and integration is that of people's open versus defensive functioning. Openness versus defense can be manifest in various ways, but in social interactions it entails being honest, transparent, trusting and receptive, whereas defense in contrast entails concealment, distrust, and often self-distortion of experience (Hodgins \& Knee, 2002). Investigating openness versus defensiveness using a diary methodology, Hodgins et al. (1996) found that people with a more autonomous orientation evidenced more disclosure, trust in others, and honesty in their daily interpersonal interactions, whereas those with a controlled orientation showed lower honesty and openness.

Another indicator of openness versus defensiveness concerns the alignment or congruence between implicit and explicit attitudes and motives. Thrash and Elliot (2002) found that persons higher in trait autonomy had lower discrepancies between implicit (projective) and explicit (self-report) assessments of achievement motivation. Hodgins, Brown, and Carver (2007) found that controlprimed individuals evidenced greater discrepancies between explicit and implicit self-esteem. Weinstein, Ryan, and colleagues (2012) found that children of controlling parents showed a greater discrepancy between their implicitly assessed sexual attractions to same sex others and their self-reports of sexual orientation. This discrepancy, in turn, related to greater hostility toward gay targets, indicative of reaction formation (Freud, 1966). In other words, 
parental autonomy thwarting can foster incongruence, and the defensive behaviors it spawns. Indeed, the mere priming of a controlled orientation has been sufficient to elicit defensive responding, as indexed by the use of hostile humor (Weinstein, Hodgins, \& Ostvik-White, 2011), the avoidance of negative experiences (Hodgins, Yacko, \& Gottlieb, 2006), or avoidance of emotionally distressing information (Weinstein, Deci, et al., 2011; Weinstein \& Hodgins, 2009). Across these studies of emotional integration and congruence it seems clear that controlled people show less integrity, whereas autonomy is conducive to more integration in both attitudes and less defensive and more open functioning.

\section{Social Contexts: Developing Emotional Integration}

The development of capacities to regulate and integrate emotions in flexible and autonomous ways is assumed within SDT to be supported by responsive, need supportive caregivers. Grolnick, Bridges, and Connell (1996) explored this with 12- to 32-month-old children. They observed toddlers in two stressful situations: waiting to play with an attractive toy (or to eat a food) and having a brief separation from mother. The children's emotionality (upset) and strategies to regulate this upset were coded. Results supported an autonomy continuum in that the more autonomous strategies were associated with the least upset, whereas the more controlled or passive strategies were associated with the most upset. Further, there were increases with age in the use of autonomous, proactive strategies, indicating development of emotion regulation through these toddler years.

As noted, the regulation of emotions and impulses can be done in a fairly controlling way. Some children, when in a negatively arousing situation such as being asked to delay gratification, exert great effort, forcing themselves to push emotions out of their minds. But controlling themselves in this way requires attention and energy, which can diminish the capacity for adaptive engagement with the environment. When used as a chronic way to deal with distress, it leads children to experience being controlled by their own harsh thoughts and to display nonadaptive engagement. In contrast, other children in the same stressful settings may be more flexible (i.e., less rigid and pressured) in dealing with the situation, for example by doing alternative activities or expressing their disappointment or frustration, signifying that they have developed more adaptive capacities for both accessing and regulating emotion.

This developing capacity for emotional regulation is not just a function of time and maturation. In fact, it is very much an interpersonal process. It is in the interpersonal realm, within which caregivers regulate children's expressed emotions more or less responsively and effectively, that children learn to regulate their own emotions. Autonomy-supportive interpersonal contexts are theorized within SDT to afford access to and expression of emotional experience, and to facilitate more reflective engagement and executive guidance of subsequent behavioral regulation (Grolnick et al., 1996; Gross \& John, 2002). Calkins and Johnson (1998) found evidence for this in demonstrating that mothers' use of positive guidance (akin to an autonomy-support approach) was associated with greater use of distraction and constructive coping in emotion inducing situations. On the other hand, children of mothers who used high levels of negative control during free play spent more time orienting to a desired but forbidden stimulus. They used less self-distraction and were less physiologically well regulated during a waiting situation relative to those of mothers who used less negative control.

Grolnick, Kurowski, McMenamy, Rivkin, and Bridges (1998) also examined strategies mothers use to help their young children regulate distress. Children of mothers who maintained their active assistance beyond what the children needed were less able to regulate their distress when on their own. Thus, while responsiveness to distress was important, parents who took responsibility for regulating children's distress and did not allow the children opportunities to self-regulate appeared to undermine children's self-regulatory capacities. Because the capacity to effectively modulate distress is so integral to mental health and the prevention of psychopathology, an environment supporting this capacity is of crucial importance.

More recently, Roth, Asssor, et al. (2009) showed in a sample of ninth-grade adolescents that parents who made use of conditional regard when adolescents expressed negative emotions (e.g., showing less acceptance and positive regard) had children who more frequently engaged in suppressive regulation of emotions, reported greater dysregulation of emotions and more resentment vis-à-vis the parents. In contrast, both mother and father autonomy support related to children showing greater integrative regulation of negative emotions. These adolescents allowed their emotions to be more fully experienced and could then, with a greater sense of choice, decide whether and how to express them. Along similar lines, Roth, Ron, and Benita (2009) showed that parents' use of conditional regard related to reduced disclosure of difficulties at school among 14-16-year-old teenagers. Presumably, with contingently regarding parents, children tend to keep the encountered problems for themselves rather than opening up, as they anticipate critical reactions from their 
mothers. A longitudinal study of adolescents by Brenning et al. (in press) further supported these associations. They found that parental autonomy support predicted increases in emotional integration and decreases in suppressive emotion regulation, whereas higher levels of emotional dysregulation were related to lower perception of parental autonomy support.

Further, Roth and Assor (2010) showed that the costs of a controlling parenting approach are apparent by the ages of five or six. Parents reported the degree to which they used both conditional regard with their children to socialize them regarding regulation of sad emotions. These parent reports were then related to various assessments of their children's emotional skills. Results indicated that parental use of conditional regard was related to children being less aware of sad feelings in themselves, less able to recognize sad feelings in other children, and less empathic in responding to other children who felt sad.

Although parents' display of contingent regard for showing negative emotions may lead them to suppress their negative emotions, it is also often the case that some parents may pressure their children to express (rather than withhold) negative emotions (Roth \& Assor, 2012). Although such a practice may seem benign at first sight because negative emotions get shared, the pressure to share negative emotions to gain parental appreciation may come at the cost of adequate emotion regulation. Consistent with this, late adolescents experiencing conditional regard to express negative emotions reported more emotional dysregulation, which hampered their capacity for intimate relations with romantic partners. In contrast, an autonomy-supportive approach was related to more integrative emotion regulation.

\section{Summary}

Developing the capacities for respecting others and delaying gratification represents a central agenda from toddlerhood through adulthood; it involves learning when and how it is reasonable and appropriate to express one's feelings and being able to use that information in a way that enhances the autonomous regulation of behavior. Thus, emotional regulation entails children internalizing caregiver-provided values and regulatory structures to manage their internal urges - a process that, similar to the internalization of behavioral regulations for uninteresting tasks, is facilitated by supports for the children's basic psychological needs. Although differences in autonomous functioning contribute to a greater congruence between felt emotions, held thoughts, and enacted behaviors, differences in contextual need support also played a role.
Previous studies suggest that controlling parental practices regarding emotions, whether involving pressure to keep negative emotions to oneself or to express them before one is ready to do so, come with costs. In contrast, need-supportive parents respect the children's rhythm and pace of expressing emotions, displaying an active interest and concern with what is emotionally salient for them. They also set appropriate limits on behavior while allowing adequate expression of feelings. This facilitates the children's attunement to the social world, and acceptance of self in the process of adapting to it.

\section{Identity Formation}

\section{An SDT View of Identity and Self}

Identity formation is among the most formidable of developmental challenges (Kroger \& Marcia, 2011; Soenens \& Vansteenkiste, 2011). This is especially so in modern cultures within which apparent options for identities are greater than ever before and in which there are fewer of the explicit vertical constraints that have historically channeled people into preordained roles and identities (Ryan \& Deci, 2011). For many scholars this fluidity and opportunity for choice represents a potential for persons to be better matched in their life roles with their interests and sensibilities (see Appiah, 2005), whereas other scholars emphasize the psychological burdens that such latitude and choice present to developing individuals (e.g., Schwartz, 2000). Both positions have substantial evidence in their favor.

Identities are self-representations-they are what and who people understand and describe themselves to be Once adopted, maintaining and defending identities can be a strong motivator of behaviors, and can orient people's learning, behaviors, and friendships (Vallerand, 2010). Different types of identities can be distinguished (Vignoles, Schwartz, \& Luyckx, 2011), with some being more personal (e.g., personal interests), others being social in nature (e.g., roles), and still others being collective in nature, thereby reflecting the groups and institutions with which we are affiliated, and supplying varied venues through which people can be recognized and valued.

Particularly during adolescent and young adult years, a challenging task is to form a meaningful identity that will serve to organize and guide an adult life. This is a period when people are particularly vulnerable to external forces. With the emergence of adolescent egocentrism and self-consciousness (Elkind, 1967), teens tend to see themselves in ways they imagine others see them, and they show a greater tendency to conform in an effort to be accepted by others. Thus, as they are attempting to formulate a sense 
of their own identity, they are also struggling with real or imagined social pressures from many sources including, not only peers but also parents, teachers, role models, and advertisers. As adolescents develop they must work to explore, try on, and accept or reject possible identities, sometimes painfully. During this process, pressures from parents and other socializers can detract the young people from smooth identity formation insofar as the offered identities are incongruent with their developing and abiding interests and sensibilities. Indeed, all people, but perhaps especially adolescents, are today exposed to the wide reach of media, which attempts to orient them toward particular types of identities and affiliations (Dittmar, 2011; Kasser, 2002). Advertisers work to have people define themselves in terms of the brands they purchase and the media cultures they engage.

Central to the process of forming identities is people's inherent desire to experience solid connectedness to individuals, groups, or cultures. By accepting the values, mores, missions, attitudes, and behaviors of others, people do not feel alone. Rather, they feel part of a group, experiencing a sense of relatedness to others and a place in the social order (Baumeister \& Leary, 1995; Ryan, 1993). Further, identities can also support the need for competence. Often people orient toward identities - perhaps being a painter or physicist, for example - that require skill acquisition, offer optimal challenges, and allow them to feel effective. Finally, the formation of identities can fulfill people's need for autonomy if they engage the relevant activities with a sense of choice and as an expression of their own values and interests. In short, people tend to gravitate toward those identities that allow maximal satisfaction of their basic psychological needs (Luyckx, Vansteenkiste, Duriez, \& Goossens, 2009)

Need satisfaction then serves as the energetic basis for anchoring identities within one's sense of self. Thus, although the terms identity and self are often used interchangeably in the identity literature, they get clearly differentiated within the SDT perspective. That is, although we have multiple identities, each is more or less internalized and integrated within the self (Soenens \& Vansteenkiste, 2011; Ryan \& Deci, 2011).

\section{Anchoring Identities Within the Self}

SDT suggests that there are two interrelated means through which identities form and become integrated to different degrees within people's self-functioning: (1) through the discovery and differentiation of intrinsic interests and talents; and (2) through the internalization of values and self-representations.
People manifest individual differences in their competencies, interests, and inclinations. Some children seem inclined toward music, others toward physical activity. The forms that such inclinations take in people's lives is a function of their interactions with the social world, but having the inclinations - for music, or sports, for example - does increase the likelihood that related activities will become important parts of identity (Deci \& Ryan, 1985b). Their inclinations lead them to explore different options and, over time, to gravitate toward others with similar interests, such that the activities, values, and relationships associated with those interests become central aspects of their lives and come to define who they are. They also are likely to experience greater competence, further enhancing their interests in the activities. In other words, people are fortunate when they adopt identities that engage intrinsic motivation (Krapp, 2002).

Still, for identities to develop out of intrinsic interests often requires both enduring affordances and social supports. A child who is naturally inclined to be physically active but who has no access or opportunities to play sports might not develop an identity as an athlete. In short, the development of an identity depends to some extent on the cultural affordances available to the person (Erikson, 1994). Further, to form an identity as an athlete will require the experience of need supports in relation to the engagement of sport activities. Supports for autonomy, competence development, and relatedness in sport will be critical in facilitating identity formation as an athlete. This is the same for identities in every domain, whether it be academic, religious, family, or career related.

Few identities are sustained solely out of intrinsic interests. Instead most are taken on or elaborated through processes of internalization. Importantly, however, much like behavioral regulations, identities can differ in the degree to which they are integrated with the self. When identities are adopted within the context of pressure and control, it is likely that they will be introjected. That is, people will take them on as "shoulds", or as identities they must enact, which essentially means that they will be identity deficits. Often in college settings, for example, there are individuals who identify as pre-med because they have always been told they will be a doctor, regardless of their interests or predilections. In fact, the students' sense of worth and esteem may depend heavily on the enactment of such an introjected identity, leading to a defensive adherence to identity and inner conflict when failing to engage in identity-consistent behaviors. Nonetheless, for identities to be not just adopted, but also autonomously engaged, the individuals will need to integrate the identities with 
their other available identities, values, beliefs, behaviors, and interests, which will allow them to be both authentic and vital in their pursuit.

Soenens, Berzonsky, Dunkel, Papini, and Vansteenkiste (2011) assessed late adolescents' identity commitments, and their autonomous and controlled motives for committing to particular identities. Even after controlling for the strength of identity commitments per se, those commitments that were autonomously motivated were associated with more positive adjustment, whereas controlled identity commitments were predictive of poorer adjustment. Thus, although adolescents could commit themselves to particular self-representations (e.g., musician, basketball player), such commitments must be accepted and integrated to ensure adjustment.

\section{Fostering Identity Development: The Role of Need Support}

Given that identities may involve intrinsic motivation, and may be anchored by either introjected or more integrated regulations, it becomes clear how a need-supportive environment is important in the process of identity formation (Soenens \& Vansteenkiste, 2011). As Assor (2012) pointed out, parents vary in the extent to which they foster inner valuing and allow adolescents to explore different interests and values, thereby nurturing self-discovery. To the extent such need supportive practices are provided, children and teenagers are more likely to develop an inner compass when making critical identity-related decisions. In contrast, finding life roles and self-definitions that are congruent with interests and integrated values is more difficult in controlling contexts. Consistent with this, Luyckx, Soenens, Vansteenkiste, Goossens, and Berzonsky (2007) showed that college students who perceived their parents as using psychologically controlling strategies were more inhibited in making identity-related decisions and in truly endorsing and identifying with the identity decisions being made.

Importantly, the development of an integrated set of regulations is presumed to be critical for subsequent need satisfaction, such that a positive spiral would develop. Evidence for such a model was obtained in longitudinal research on high school and college students by Luyckx et al. (2009), who focused on the extent of exploration, commitment, and ruminative thought these students displayed with regard to the directions and identities they were adopting. Results showed that satisfaction of SDT's basic psychological needs was in general positively related to making and identifying with particular identities, and to enhanced breadth and depth of identity explorations. Those with less consolidated identity commitments and those associated with more rumination had lower basic need satisfaction.
Moreover, cross-lagged analyses provided evidence for reciprocal relations - need satisfaction both facilitated more positive identity formation, and more positive identity formation facilitated greater need satisfaction.

Although there are clear advantages to integrating identities, some identities are socially stigmatized, and some may conflict with other identities or roles to which a person aspires. Such identities often remain poorly integrated. These identifications can be resisted, or remain relatively isolated or compartmentalized, as opposed to being integrated with other identities, needs, and value attachments of the self (Ryan \& Deci, 2011). For instance, suppose in his political considerations a man identifies with an ideology that is oppressive to certain minorities, and yet in his religious considerations he endorses being a generous and loving human being. Both might be values that he deems important, but their inconsistency requires that he keep them compartmentalized. Advocating harm to others might catalyze guilt or uncertainty when he is in his more religious identity. Yet integrating these might require change in one or both identities, and the psychological threat or loss of relatedness that might accompany such change.

The more autonomy supportive one's social context, the more easily the person can integrate identities and self-characteristics. For example Weinstein, Deci, et al. (2011) examined how autonomous versus controlled motivation, operationalized through either individual differences or the semantic priming of autonomy versus control, facilitates or inhibits the integration of positive and negative past identities. Results of their studies showed that more autonomously motivated participants felt closer to, and were more accepting of, both negative and positive past characteristics and identities, whereas more controlmotivated participants were closer to and more accepting of only their positive, but not negative identities and characteristics. Moreover, defensive processes, as reflected in the use of nonpersonal pronouns and stronger escape motives, mediated these effects, indicating that lower defensiveness allowed fuller integration of negative identities. Finally, results demonstrated that when participants did more fully integrate both positive and negative past identities, they exhibited greater vitality and sense of meaning.

\section{Toward Qualitatively Different Types of Identities}

Finding identities that are need satisfying is often a struggle, as many social contexts pressure individuals to adopt identities that may or may not befit their interests or talents. Social contexts also can reject or demean other identities and roles. Thus, identities can be adopted for 
controlled reasons, with the predicted negative outcomes for both well-being and persistence.

Identities can also be motivated as a response to need thwarting. SDT argues that, when needs are strongly thwarted in development, persons may adopt need substitutes referred to as extrinsic aspirations, such as those involving the pursuit of materialism, an appealing outer image, or ideas of fame and popularity (Kasser \& Ryan, 1996). These identity pursuits often serve a defensive function, blocking feelings of vulnerability, and often are pursued to compensate for a lack of support and acceptance from others during development. In line with this, Kasser, Ryan, Sameroff, and Zax (1996) predicted that identities focused on materialistic life goals might be associated with (and compensating for) experiences of autonomy and relatedness thwarting from caregivers. Using a longitudinal dataset, they showed that adolescents whose mothers were less warm and autonomy supportive when the children were 14 years old were more materialistic at age 18 .

Adolescents who experience need thwarting may be especially vulnerable to extrinsic ideals promoted within the media and advertising, which often suggest that the pursuit of extrinsic ideals will yield the confidence, love, or self-esteem such teens do not feel. For example women high in autonomy frustration may be more susceptible to ambient extrinsic ideals for thinness and selfobjectification. Mask and Blanchard (2011) examined this by having individuals differing in general levels of autonomy watch and react to a video depicting the thin ideal. After watching the video, women low in autonomy reported greater pressure to be thin, more dissatisfaction with their bodies, and increased concern with the quantity of food they ate. In contrast, women high in autonomy became more concerned with the quality of the food they ate, but they did not feel increased pressure or selfdissatisfaction (see also Pelletier, Dion, \& Levesque, 2004) Thus, to the extent adolescents have built up a stronger inner foundation, they can rely on it to more autonomously decide how to handle identity pressures, which suggests that autonomy is an important factor in resilience.

\section{Summary}

We have discussed how the formation of identities and self-representations that are well internalized and integrated represents a major challenge for contemporary adolescents. Research suggests that without sufficient contextual need support, adolescents' development of a strong inner compass (Assor, 2012), and the adoption of identities truly grounded in their emerging interests and personally held values, may be forestalled.

\section{AUTONOMY DISTURBANCES IN DEVELOPMENT AND PSYCHOPATHOLOGY}

\section{Need Frustration and Psychopathology}

The development of the self proceeds most optimally in social contexts where children experience ongoing supports for their basic psychological needs. When needs are supported in domains and activities appropriate to their developmental-level children display increasing amounts of autonomy. The situational and cumulative effects of support for psychological needs are expected to yield inner resources in the developing persons that further contribute to subsequent wellness and adaptation. Yet often development does not proceed optimally, especially when social contexts thwart the children's basic needs and in doing so amplify vulnerabilities and potentiate psychopathology.

Many forms of psychopathology entail impairments of autonomy, as failures in organismic integration and in the development of self contribute to a range of dysfunctions. From the perspective of SDT, explicating autonomy impairments involves a consideration of the interplay between the psychological needs for autonomy, competence, and relatedness that support the developmental processes of attachment, intrinsic motivation, internalization, emotional regulation, and identity formation, and the social contexts, such as parent or institutional behaviors, that either support or thwart the satisfaction of these basic psychological needs. In what follows we focus on specific types of disorders, both in terms of a theoretical account of autonomy impairments and a review of relevant research. Specifically we discuss three general categories of psychopathology, all of which involve serious autonomy disturbances but each of which has particulars forms of autonomy impairments, leading to one of the categories of pathology, as is graphically illustrated in Figure 9.2.

First, we consider disorders in which internally controlling regulation of behavior predominates. These are disorders that, despite their many differences, are characterized by the salience of introjection or rigid internalizations and the associated feelings of anxiety, self-criticism, and threat. We especially focus on obsessive-compulsive disorders, paranoid personality, self-critical depression, and controlled eating disorders (i.e., anorexia and bulimia).

A second type of autonomy disturbance concerns impairments in internalization of social values and regulations, characterized by difficulties in the self-regulation of behavior. To illustrate these types of autonomy disturbance, we discuss problems of impulsivity and aggression, including oppositional defiance, conduct, and antisocial 


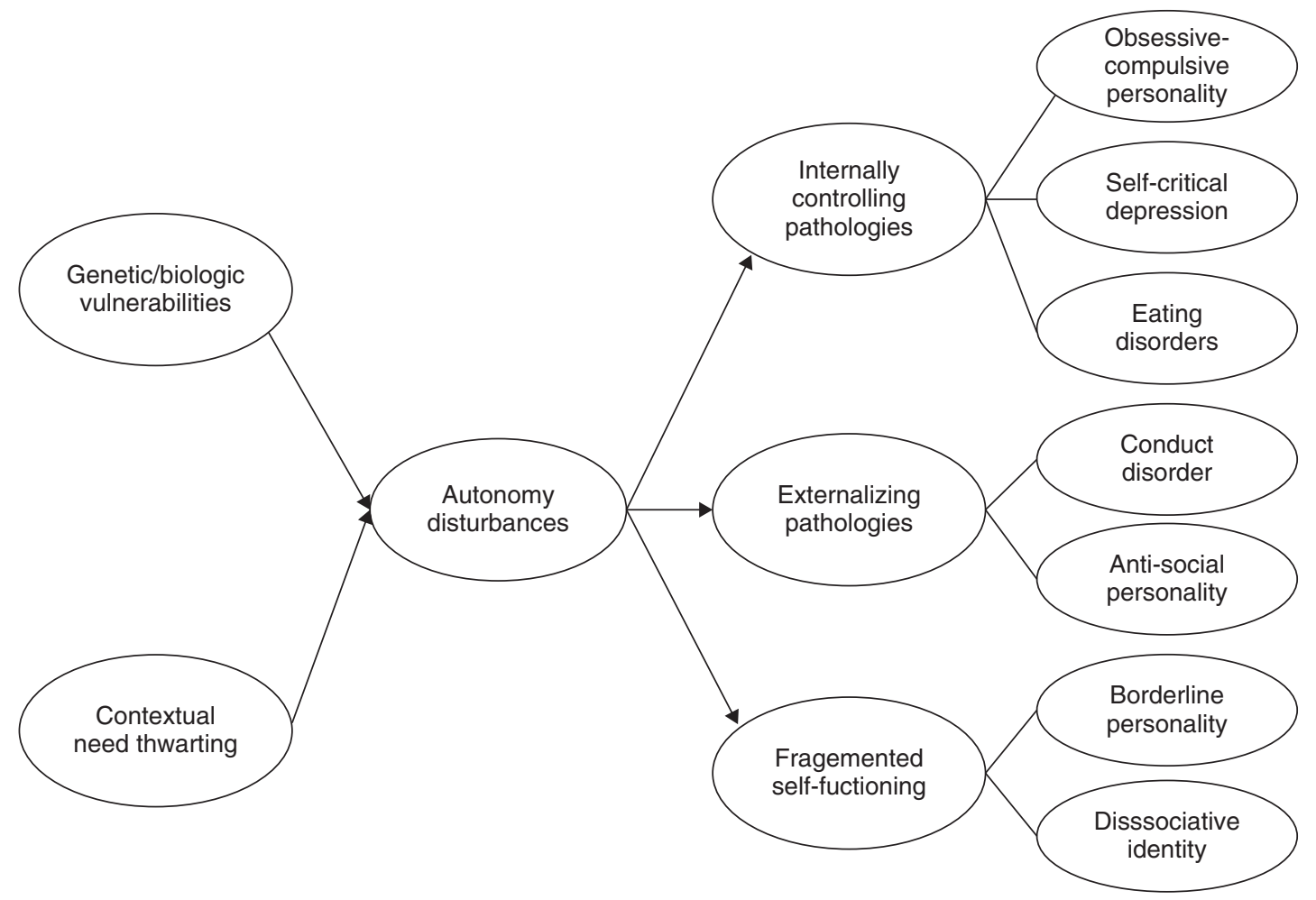

Figure 9.2 Graphic overview of selected psychopathologies reflecting autonomy disturbances.

personality disorders. A third category of autonomy disturbances we discuss are disorders associated with more severe thwarting of psychological needs in development, namely disorders associated with fragmented self-functioning. Here we focus especially on persons with borderline personality disorder and dissociative identity disorders, and on the integrative difficulties posed by controlling, inconsistent and intrusive others who thwart basic psychological needs and damage self-development.

In discussing each of these types of autonomy disturbance we address the experience of autonomy within each, and discuss factors relating to the social contexts of development that may contribute to impairments in autonomy and integration. Although it is the case that any factor that disrupts the organizational tendency, whether biological, interpersonal, or cultural in nature, can potentiate disturbances of autonomy, in many cases the sources of disrupted autonomy can be strongly related to deficiencies in the social environment - that is, to its failure to provide appropriate autonomy support, structure, or involvement (Ryan \& Deci, 2000b; Vansteenkiste \& Ryan, 2013). Yet even in those cases where the causes of the disorder do not lie in need thwarting contexts, the symptoms associated with such impairments can themselves interfere with autonomous functioning, and are often experienced as not under volitional control. In short, we discuss autonomy issues both as both an etiological factor and as a symptomatic factor, in varied forms of psychopathology.

\section{Disorders Involving Introjection and Internally Controlling States}

There are rich and variegated literatures concerning internalizing disorders, in which a person is beset with internal pressures, judgments, compulsions, and other self-directed mandates to act. These disorders, associated with both anxiety and depression, centrally involve a lack of autonomy and volition, as often motivations to act are highly controlled and even coercive. This literature also indicates that caregiver styles characterized by a lack of autonomy support and the presence of controlling or authoritarian strategies contribute to internalizing disorders, especially in vulnerable children (e.g., Bayer, Sanson, \& Hemphill, 2006; Muhtadie, Zhou, Eisenberg, \& Wang, 2013). What SDT's perspective adds to this literature is a rich body of findings concerning how controlling environments foster the development of internally controlling regulations - that is, how such conditions lead to regulation through introjects (Deci \& Ryan, 1995; Ryan \& Deci, 2008). 
Introjects are understood within SDT as motivations characterized by internal pressure, enforced by contingent self-worth. Introjection involves an ongoing salience of strict internalized demands. Such demands and standards are introjected in the sense that one's motivation is based largely on internalized self-related emotions and appraisals. In introjection people adopt external standards as their own and determine self-worth based on whether they meet them, without making the standards a part of their integrated sense of self. Often the process of introjection entails a type of self-deception in which the people thinks they want what the authority originally prescribed (e.g., Weinstein, Ryan, et al., 2012). This is akin to what Winnicott (1960/1965) referred to as false self, in which cognitive functions gradually lose their grounding in organismic processes. Yet heteronomy is phenomenally manifest in the pressured, have-to nature of experience, backed by threat of anxiety and self-criticism that drives much of the person's behavior. Introjects can indeed be highly motivating, and represent the core driving forces in people's lives. Thus, even when not formally diagnosed, the dynamics of introjection can catalyze significant inner distress. In addition, when rigid, punitive, or overly pervasive, they can be highly dysfunctional. This is particularly true for persons who are diagnosed as having an internalizing disorder.

Although, as we shall stress, the etiology, presentation, and course of internalizing disorders are complex and multidetermined, evidence that caregiver styles can contribute to such problems is also relatively clear. For example, consider that factors such as loss of parents (and thus of attachment supports), depression in parents (and thus low involvement or autonomy support), and excessive parental control (thus thwarting autonomy needs) have all been implicated in the development of childhood depression and self-critical styles (Ryan et al., 2006). SDT more specifically relates the formation of introjects to the thwarting of autonomy and relatedness needs, a process central to a variety of clinical presentations (Ryan et al., 2006). Thus, we look at parental thwarting of autonomy and relatedness as factors in internally controlling disorders.

\section{The Obsessive-Compulsive Disorders}

Two separable diagnoses carry the rubric of obsession and compulsion: obsessive-compulsive disorder (OCD) and obsessive-compulsive personality (OCP). These distinct entities share some common features in the dynamics of behavior regulation, but they also differ in meaningful ways.
OCD is characterized by the experience of intrusive thoughts and demands that typically can be alleviated only by engaging in ritualistic, rigid behavior. The thoughts are often ego-dystonic, in that they are unwelcome and anxiety provoking, and thus such thoughts are experienced as having an origin outside the self. Indeed, these thoughts often are inconsistent with the person's conscious values and ideals. An example is an OCD-diagnosed woman who reported continual intrusive thoughts concerning hurting her baby whenever she was near certain objects. These intrusive thoughts raised considerable anxiety precisely because they ran against her strongly held desire to keep her child safe. To alleviate this anxiety she was compelled to check continuously for dangerous objects in her vicinity. OCD is classified as an anxiety disorder precisely because obsessions and rituals often have the function of regulating anxiety, albeit usually with only temporary success.

An important feature of the dissonant thoughts often found in OCD is that they are persistent, unwanted, and difficult to control. From the perspective of the individual the unwanted thoughts befall him or her and are not autonomous or volitional. The person then feels coerced or compelled into ritualistic behaviors; he or she must do them or face dreadful anxiety. The actions that alleviate obsessional thoughts therefore have an external perceived locus of causality. As an aspect of this external causality, compulsive behavior patterns typically are performed under strict constraints - there is an inner demand to engage in actions in rigidly prescribed ways. These orders are experienced as heteronomous, albeit within the person. The cost of failure is guilt, anxiety, and self-disparagement, and in other cases a sense of panic and fragmentation of the self. The regulatory process of compulsive acts is thus accompanied by a sense of inner pressure that we would describe as internally controlling. That is OCD has the regulatory form of introjection, even though it may not always have social roots

Like OCD, OCP is characterized by living in a very structured and orderly way, which can have both positive and negative consequences depending on how severe it is. In less severe cases, the regulation of behavior is stil introjected and thus has the negative psychological consequences associated with introjection. Nonetheless, the order that is manifest from mild OCP can have adaptive aspects, for example in getting homework done or organizing possessions in orderly ways. Yet OCP is often more severe and compromising, with debilitating pressure and anxiety, and behaviors that are rigid and extreme. For example, people's collecting which is likely to be based in mild OCP is likely to become hoarding when the pathology becomes more serious, and their organizing may become 
intensely rigid with threats of self-disparagement and selfpunishment hanging over their heads to keep them in line.

Unlike OCD, there appears to be a less robust biological contribution to OCP, even though there are some common symptom dynamics. Supporting the differential role of biology, results by Jenike as early as 1991 found responsiveness among OCD patients to pharmacological interventions, but not among those with OCP. Also OCP, in contrast to $\mathrm{OCD}$, is more a lifestyle than a symptom, and in many ways OCP can be viewed as a continuum from a somewhat rigidly focused set of attitudes to a disorder that interferes with ongoing functioning. Accordingly, many features of this controlled lifestyle are ego-syntonic rather than disturbing (Sperry, 2003). For example, individuals with OCP who organize their possessions and become distraught when things get moved or misplaced might go into treatment not because they view the orderliness as problematic, but because their pattern of living, which seems fine to them is causing interpersonal or vocational difficulties. In addition, as reported by Othmer and Othmer (2002), persons with OCP often display their characteristic drivenness, compulsiveness, and inflexibility when events involving authority, intimacy, or lack of structure trigger them. Nonetheless, the surface structure of these behaviors share similarities with the controlled behaviors so often manifest in OCD.

The internally controlling nature of regulation in $\mathrm{OCP}$ can, in some instances, be highly stable and self-sustaining, in part because it can come with ongoing external approval and derivative internal gratifications. For example, Assor and Tal (2012) examined adolescents' perceptions of their mother's use of parental conditional positive regard (PCPR) to motivate them to academically achieve. Those teens whose mothers applied contingent positive regard evidenced more self-aggrandizement following successes yet greater self-devaluation and shame following failures. This hypersensitivity to outcomes in turn predicted compulsive over-investment and maladaptive self-feelings, even when controlling for the effects of conditional negative regard. Thus, it appears that the experience of maternal conditional positive regard to promote school achievement leads to self-esteem dynamics in which the adolescents vacillate between feelings of grandiosity following success and self-derogation and shame following failure, representing significant emotional costs. These general population findings are reminiscent of the distress many OCP patients vividly describe.

It is particularly clear with OCP that the pathology of obsessive-compulsive actions is not always defined by the behavior itself, because as noted the behavior can be effective and functional. Rather, it is the rigid regulatory processes underlying behavior that are dysfunctional. Persons with OCP, while not acutely afflicted with intrusive thoughts sometimes befalling those with OCD, are often inordinately concerned with carrying out the actual or presumed demands of authority. For example, a person with this personality style can be quite industrious and fastidious in complying with introjected demands, and this may yield high activity and even productivity. However, rigidly controlled workaholics, who are likely to have OCP, tend to evidence a range of ill-being consequences (Van den Broeck et al., 2011). Clearly, the industriousness and rigidity of people with OCP is quite different from the vitality and spontaneity that characterizes autonomously functioning individuals, even ones who work excessively. Ryan and Deci (2008) argued that vitality concerns the free energy a person has at his or her disposal. The internally controlled regulation of an individual with OCP is often experienced as energy draining and without joy. As Sperry (2003) noted, the demeanor of someone with OCP can be "grim and cheerless" (p. 178). Moreover, resistance to inner dictates, when possible, may feel like a gargantuan effort.

There is an interesting point about OCP that highlights an important aspect of its disturbed autonomy. Although obsessive-compulsive individuals often act with determination in carrying out introjected dictates, they also sometimes display confusion and indecision. The problem especially arises when there is no dictate, no established course to follow - that is, when there is uncertainty and one needs to fall back on inner guidance. At such times, the people may be rocked with indecisiveness and ambivalence. This illustrates clearly how dependence on controlling regulations, being unintegrated, leaves one without self-direction. The anxiety of indecision highlights how the seeming determination behind many obsessivecompulsive acts is not autonomous; there is no inner compass to rely on when external direction is lacking.

Although the obsessive-compulsive disorders represent clear instances of psychopathology with disturbed autonomy, it is not clear, nor theoretically necessary that they are exclusively outcomes of parental control. Evidence has, in fact, been compelling for robust biologic contributions to OCD, whereas parental factors appear to play a larger role in the development of OCP. Indeed, case literatures concerning OCP have more frequently pointed toward controlling, often intrusive parenting (e.g., Millon, Davis, Millon, Escovar, \& Meaghan, 2000). Benjamin (2003) suggested that persons with OCP often come from demanding, even coercive parenting environments. Moreover she argued that the controlling emphasis is more often 
on punishment for failures than on acknowledgement for success. Yet, here too, systematic investigation of the social context of development of OCP individuals is not extensive. It does appear, however, that the controlling regulatory styles of those with $\mathrm{OCP}$ are more likely to be linked with excessive parental controllingness than is the case for OCD.

\section{Introjection and Self-Disparagement: Self-Critical Depression}

At the core of a number of pathologies are introjected demands that organize intentional behavior. Although this type of autonomy disturbance involves conflict and tension, individuals with rigid character are often able to behave intentionally and satisfy their introjects. There are other types of introjected demands, however, that individuals cannot easily attain, and these invariably result in experienced failure and self-disparagement. These forms of disturbed autonomy, like those of rigid character, begin with individuals' introjecting the demands of authorities and basing their self-worth on living up to those demands But here, the pathology and experience are quite different, for the individuals' predominant experience is failure and worthlessness. Thus, not only is their autonomy undermined by introjects and the social conditions that instilled them, but they also feel incompetent and unloved by significant others. Two such pathologies of introjection are self-critical depression and eating disorders. We focused first on self-critical depression.

Research on depression and its etiology has increasingly pointed toward two distinct pathways to the disorder, each of which can build on biological vulnerabilities (Luyten \& Blatt, 2013). The first is concerned primarily with internalization of excessive demands for achievement and is characterized by harsh self-criticism and guilt, and the second is concerned more with the loss of relatedness, love, or attachment. Although both of these vulnerabilities involve self-esteem dynamics, and issues of autonomy and relatedness are intertwined in both, the former, self-critical form of depression is particularly relevant to our discussion of autonomy disturbances, internalization, and internally controlling regulation. We thus focus on the self-critical depression syndrome, which has been labeled variously as a disorder of achievement-autonomy (Neitzel \& Harris, 1990), introjection (Blatt, 1974), and autonomy (Beck, 1983).

By whatever label, this syndrome involves a type of disturbed autonomy in which people experience dysphoric affect and lethargy resulting from the belief that they are failures. In this disorder there are rigid standards or ideals that have been introjected, along with the belief that failure to attain them means they are unlovable and unworthy. Thus, the punitive introjects, with their ties to contingent self-worth, leave people vulnerable to ongoing self-scrutiny. Because with such introjects there is little individuals can do that is good enough, they will invariably experience a sense of worthlessness. In other words, self-disparagement tends to be ubiquitous. Individuals with such introjects are harsher in their self-judgments than in their judgments of others, and these self-judgments often form the precursors to depressive episodes (Beck, 1983). Their harsh self-judgments may even manifest outwardly, through engagement in nonsuicidal self-injurious behaviors, which reflect the scars of the inner critic (Claes, Soenens, Vansteenkiste, \& Vandereycken, 2012). Accordingly it is hardly surprising that adolescent depression and low self-esteem are associated with low autonomy (e.g., Noom, Dekovic, \& Meeus, 1999).

The phenomenological functioning underlying selfcritical perfectionism is particularly relevant to the understanding of depression as involving disturbed autonomy. With respect to significant self-goals people see themselves as responsible yet as incapable. Thus, the absence of felt competence to attain internalized goals results in a sense of amotivation (Deci \& Ryan, 1985a). At the same time many of the specific demands on the self to achieve or succeed have the character of have-to and must revealing their phenomenological character as having an external perceived locus of causality (i.e., as being heteronomous with respect to the self). Consistent with such an analysis, self-critical perfectionism has been found to be associated with more controlled functioning (e.g., Miquelon, Vallerand, Grouzet, \& Cardinal, 2005) and increased psychological need frustration over time (Boone, Vansteenkiste, Van der Kaap-Deeder, Soenens, \& Verstuyf, 2014).

There has been recent interest in the development of perfectionism, a characteristic related to self-critical depression. In line with our argument that such internally controlling characteristics reflect disturbed autonomy, evidence suggests that individuals with high levels of self-critical perfectionism frequently report having experienced harsh and authoritarian parenting (see, e.g., Flett, Hewitt, \& Singer, 1995; Frost, Novara, \& Rheaume, 2002; Kawamura, Frost, \& Harmatz, 2002). For example, Enns, Cox, and Clara (2002) found support for a model in which harsh parenting led to maladaptive perfectionism, which in turn was associated with an increased proneness to depression.

Such findings suggest perfectionism as a pathway through which controlling environments might increase 
depressive symptoms. Consistent with this, investigations of the backgrounds of self-critical depressives point to controlling parental styles. In one illustrative investigation, McCranie and Bass (1984) found that women high in self-critical depression had parents who maintained strict control, demanded high achievement, and were inconsistent and contingent in their conveyance of love. Whiffen and Sassville (1991) reported similar results for both males and females. Research based in SDT concurs, with longitudinal data showing that children growing up in controlling families are more likely to adopt self-critical perfectionistic attitudes, which, in turn, relate to elevated depressive symptoms (Soenens, Luyckx, et al., 2008). Moreover, the adoption of self-critical perfectionistic standards in controlling environments may not elicit depressive symptoms but may also involve self-esteem deficits (e.g., Soenens, Vansteenkiste, Luyten, Duriez, \& Goossens, 2005)

Empirical findings are thus consistent with our speculations concerning the nature of the social contexts leading to introjection and internally controlling states. In this frame one can view self-critical depression as a chronic and pervasive state of ego-involvement in which one continuously fails to live up to the demands and is thus punished. Unfortunately, self-critical perfectionism appears to get transmitted from the one generation to the other through controlling parenting (Assor et al., 2004; Soenens, Elliot, et al., 2005). Presumably, the contingent love self-critical perfectionists display vis-à-vis their own functioning gets projected onto their children, such that children experience their parents as displaying conditional regard, which subsequently affects their own functioning.

Indeed, such dynamics between parent and child may be mutually problematic. Using a cross-lagged longitudinal design in two samples of middle and late adolescents, Soenens, Luyckx, Vansteenkiste, Duriez, and Goosens (2008) compared three models concerning the role of parental control in depression. Results generally favored a reciprocal model in which controlling parent behaviors contribute to increases in depression, which in turn enhance parents' tendency to control their teenager. This represents a truly maladaptive cycle between parent and child. This bidirectional relationship suggests that more depressive symptoms in an adolescent can elicit less autonomy support from parents over time, even as autonomy support from parents conduces toward fewer depressive symptoms in their adolescents.

Further, Soenens, Park, Vansteenkiste, and Mouratidis (2012) examined whether this association between parental control and depressive symptoms among adolescents might be culture bound as some cultural relativists have argued, versus whether as SDT suggests, the detrimental effects of psychological control and the loss of autonomy generalize across cultures. Soenens et al. (2012) specifically investigated the relevance of two domain-specific expressions of psychological control (i.e., dependence oriented and achievement oriented) in both Belgian and South Korean adolescents. Analyses showed very similar relations between the psychological control, depressive personality, and depressive symptoms across the two samples, thus supporting the notion that the effects of psychological control generalize across cultures.

In short, there are clear relations between control and these internally controlling forms of depression in which self-critical cognition is salient. Although there is substantial evidence that vulnerability to depression varies, the importance of autonomy thwarting in this expression of psychopathology is becoming ever better documented.

\section{Eating Disorders: Anorexia and Bulimia}

In her classic book on the topic, Bruch (1973) described eating disorders as pathologies of autonomy. She argued that these eating pathologies typically involve a struggle for control that takes the form of an obsession with eating and body image, with control over one's body dynamically staving off a pervasive sense of ineffectiveness. Bruch saw people with eating disorders as exemplifying the capacity, as a function of harsh introjects, to manipulate bodily states and desires to preserve the illusion of self-sufficiency and to feel a sense of control with respect to oneself and others. In restrictive anorexia, the introjects around eating and weight are more stable and effective in keeping people's eating behaviors in abeyance, whereas in bulimia there is a more open conflict between rigid controls and akratic eating. Bulimics engage in binge eating - in expressive, uncontrolled consumption - only to be plagued by guilt and self-derogation leading to vomiting or abuse of diuretics.

The psychodynamics highlighted by Bruch were empirically supported in an early study by Strauss and Ryan (1987). They found greater self-oppression and selfrejection in both anorexic and bulimic participants than in a matched control group, suggesting internally controlling dynamics, whereas control-group participants displayed more flexible self-management and self-acceptance. Furthermore, Strauss and Ryan documented a particularly heightened impersonal causality orientation (Deci \& Ryan, 1985a) among restrictive anorexics, indicative of an impoverished sense of both autonomy and effectiveness. 
Internally controlling forms of regulation are readily apparent in the dynamics of many behaviors entailed in eating disorders, especially those involving restrictive self-control. Whether anorexic or bulimic these patients display inordinate concern with how others view them, and hypertrophied public self-consciousness. As Plant and Ryan (1985) argued, such consciousness potentiates an external perceived locus of causality in which people have to conform to the projected views of others. Although a focus on weight is often paramount, eating disordered patients are typically self-conscious, demanding, and self-critical with regard to many aspects of appearance and behavior. And although restrictive anorexics in particular appear to display a high degree of personal control, the regulatory basis of this control is dictatorial.

Applying SDT to anorexia, Vansteenkiste, Soenens, and Vandereycken (2005) described how a focus on thinness represents an attempt to gain a sense of security and worth, yet the thinness, even when attained, fails to supply the anticipated emotional benefits. The anorexic person is never thin enough, yet they continually work with the belief that positive feelings are a few pounds away. The paradox, however, is that achieving their extrinsic goal is satisfying to some degree, so it further anchors them in this pattern of behavior. These patients often strongly assert that they do feel better when losing weight and believe that the pursuit of weight loss is therefore not the source of distress. Indeed, their own experiences seem to confirm that efforts at weight loss are part of the solution. The transitory nature of relief thus fosters an addictive quality to the internally controlling drive for thinness

Whereas the restrictive anorexics can display a high degree of control with respect to eating, bulimic patients often find their control overwhelmed by an impulse to binge eat. Binges typically occur at times of high psychological need frustration, thus showing how their introjected regulatory structures are not sufficiently stable to keep the impulse in check. The binge impulse is often a reaction to the experience of need frustration rooted in internal criticism, so it represents an attempt to escape from the painful sense of self that carries the burdensome, introjected standards. For example, Verstuyf, Vansteenkiste and Soenens (2012) researched the connections between need frustrations and binge eating. In a sample of female adolescents, they showed indeed that an investment in appearance-focused motives led to need frustrations, which in turn predicted increased bulimic symptoms. Along similar lines, a diary study in female adolescents (Verstuyf, Vansteenkiste, Soenens, Boone, \& Mouratidis, 2013) indicated that daily variation in psychological need frustration covaried with ups and downs in bulimic symptoms, suggesting that binge eating serves to compensate for painful experience of need frustration.

It is interesting to note that, as the psychic threat gets greater for individuals with the bulimic disorder, the people's regulatory capacities becomes weakened, whereas in people with obsessive-compulsive personality the regulatory capacity often becomes even more rigid and dominant. The lack of stability of the regulatory introjects in the people with bulimia thus allows for the akratic action, but the self-evaluative introjects invariably result in self-disparagement and feelings of depression for having lost control. It is therefore interesting to note in this regard that the long-term course for many restrictive anorexics is a shift to bulimic patterns of coping.

Although again there are multiple contributors to the development of bulimia and related eating disorders the role of familial factors in setting up the dynamics of introjection and internal control are quite salient. Bruch (1979) has vividly depicted the role of parents in catalyzing anorexia by depriving their daughter of autonomy and the "right to live her own life" (p. 38). Minuchin, Rosman, and Baker (1978) similarly reported high levels of enmeshment and intrusive control in families of patients with eating disorders. Strober and Humphrey (1987) reported that both anorexics and bulimics experience parents as blaming, rejecting, and critical. Strauss and Ryan (1988) found less mutuality of autonomy in the object representations of both bulimic and anorexic participants, and lower expressiveness within their families. Another more recent study found that late-adolescent women hospitalized for eating disorders reported having more psychologically controlling fathers than a matched sample of women without the eating disorders (Soenens et al., 2008).

\section{Summary}

Each of the disorders we have considered thus far begins with rigid, demanding, and critical introjects. These rigid structures take varied forms and are more or less stable and effective in controlling the people's actions. In some cases, most notably obsessive-compulsive personality and anorexia nervosa, the disordered individuals can feel a strong sense of personal control and self-efficacy - the OCP can keep personal affairs orderly and anorexics can keep body urges under control. But these, like the other disorders involving salient introjects, constitute disturbed autonomy and can terrorize people with such contingent self-esteem. Findings in this area also highlight 
the important difference between personal control and autonomy; being self-controlled can sometimes yield desired outcomes yet not be experienced as autonomous or volitional (Muraven, Gagné, \& Rosman, 2008).

We turn now to disorders characterized not by the prevalence of introjects, but rather by the relative absence of internalization. These forms of psychopathology involve the lack of adequate regulatory structures that link one effectively to the socializing context.

\section{Impairments of Internalization in Externalizing Disorders}

As we argued earlier, the process of internalization is dependent upon certain affordances in the caregiving environment - namely, autonomy support, structure, and involvement-which together facilitate both attachment to caregivers and a readiness to assimilate the values they model. Some caretaking environments, particularly those characterized by coldness and hostility or by neglect conduce toward poor quality of attachments and lessened internalization of social norms and prosocial behaviors (Johnson, Cohen, Chen, Kasen, \& Brook, 2006; Joussemet et al., 2008). In addition, those low in autonomy supports, especially the lack of both perspective taking and attunement to needs and interests, are at risk for both low social skills and negative social interactions (Shields et al., 2001). In fact, parenting environments impact on internalization through their effects on need satisfaction (Ryan et al., 2006). Controlling parents not only restrict opportunities for children to develop self-regulatory capacities, they create emotional and need-related frustrations that can fuel negative behaviors.

In the realm of more severe forms of psychopathology, we are also concerned with more extreme forms of need thwarting, including physical, sexual, and emotional maltreatment, which thwart all three basic needs, with irradiating effects across multiple developmental lines. Such parenting conditions may result either in externalizing problems (Gershoff, 2013) such as conduct disorders, or, especially when the thwarting is extreme, in disorders such as dissociative identity.

Illustrating this with respect to externalizing disorders, Shields, Cicchetti, and Ryan (1994) examined the social-competence self-regulation impairments of children who had been maltreated by caregivers. The setting was a summer camp for both maltreated and economically disadvantaged children, allowing for a comparison of these groups. As predicted, children who had endured maltreatment displayed, as rated by observers, less social competence, more emotional dysregulation, and impaired self-regulation reflected in externalizing disorders as well as internalizing disorders. Indeed, self-regulatory deficits mediated the relations between maltreatment status and social competencies.

In a subsequent study, Shields, Ryan, and Cicchetti (2001) further explored this developmental process by assessing maltreated and nonmaltreated children's representations of the parenting they experienced. They found, as expected, that the representations of parents in maltreated children, as accessed through a projective narrative task, were less coherent and integrated, and more negative and constricted than those of nonmaltreated children. These differences in parental representations were in turn related to the externalizing processes of emotional dysregulation, aggression, and peer rejection in the ecologically valid camp setting.

These issues concerning need thwarting conditions and impairments in self-regulation have particular salience with respect to conduct and oppositional disorders, in both of which internalization, self-regulation, and capacities for relatedness are impaired.

\section{Conduct Disorders and Antisocial Personality}

Broadly speaking antisocial personality disorder (APD) applies to persons who lie, steal, manifest an impoverished sense of responsibility, are aggressive and manipulative toward others, and show evidence that these patterns are continuations of behavior disorders earlier in life. Children diagnosed with conduct disorders, a frequent antecedent of APD, display control problems, lability, a lack of ability to give and receive affection, and delayed or impaired development of conscience. Further, self-aggrandizement and egocentrism typically characterize their behavior, and their lying about accomplishments highlights their excessive need to be recognized or esteemed by others in an immediate way. Like adults with ADP, these children tend to display a lack of conscience, or deep concern with what is good or right. Related to conduct disorders and APD is the behavioral category of reactive or destructive aggression, the developmental routes of which have been related to those associated with these diagnostic categories.

Etiologic theories of APD have clearly implicated biologic or genetic factors such as poor autonomic reactivity (Burnette \& Cicchetti, 2012; Raine, 2013). Yet even with this recognition of potential biologic vulnerabilities, it is clear that such vulnerabilities become amplified in nonnurturing environments. Our contention is that APD is a psychopathology entailing impaired internalization, and although internalization may have been compromised by biological factors (Jafee, Moffitt, Caspi, \& Taylor, 2003), 
we maintain that these impairments can also be linked to, and are interactive with, deficits in the social contextual factors associated with the basic psychological need supports that are essential for internalization to develop.

An internalization perspective looks toward the family environment for the relational resources that support the internalization of values of a prosocial nature, resources that are frequently lacking in the families of persons developing APD. For example, the empirical evidence supports the idea that prosocial values are most likely to be acquired (or expressed) when caregiving is characterized by warmth (Maccoby, 1980), low power assertive discipline (Hoffman, 1960), and autonomy support (Ryan \& Hawley, in press).

The experience of growing up in a nurturing, caring, and responsive familial environment undoubtedly facilitates prosocial values both directly and indirectly. A person who grows up in a basic need supportive environment will have been exposed to models of caring and concern about others, from figures to whom they are likely to attach and desire to emulate. Support for this comes from many quarters. For example, Ryan and Connell (1989) reported that children who experience a high quality of relatedness to parents were more autonomously motivated in the prosocial domain. Conversely, a variety of clinical and empirical perspectives have suggested that antisocial personality, oriented towards self-serving, manipulative, and hedonically gratifying acts, has its roots, in part, in cold, inconsistent, and controlling family environments (e.g., Benjamin, 2003). A plethora of studies has also shown that the common backdrop to APD and conduct disorders of childhood includes such factors as maternal depression, loss, high family conflict, other parental pathology, and impoverished conditions of life that fragment the family (Coie \& Jacobs, 1993; Loeber \& Stouthammer-Loeber, 1986). All of these factors potentiate a situation ultimately lacking in the basic nutrients upon which internalization depends - namely autonomy support, adequate structure and guidance, and concerted, caring involvement.

One limitation of these models of value acquisition and their implications with regard to the development of externalizing disorders such as APD, is that they do not focus on how or why children might develop nonprosocial or nonmoral attitudes, except as a converse or absence of moral internalization. For example, there are probably very few parents who strive to teach their children to be manipulative, materialistic, or Machiavellian, but there are many children who develop such an orientation. Absence of identification does not explain why one proactively seeks to act in these ways. Thus, a fuller model of APD requires both explaining why internalization fails, and why hedonic aggressive, self-gratifying values predominate instead.

Directly informative in this regard is a six-year longitudinal study by Joussemet et al. (2008), who followed children from six to twelve years of age. Specifically, they identified developmental trajectories in childhood aggression in which they applied SDT. They found that over these years children on average engage in a lower frequency of aggressive acts, presumably reflecting socialization and development. Yet the slope of that trajectory differs among children, such that while for most there are declines in aggressive acts, for others there are maintained or escalating frequencies of aggression. After controlling for a number of other risk factors for being aggressive (e.g., being male, having a reactive temperament, parental divorce/separation), Joussemet et al. found, as they hypothesized based on SDT, that having a mother who was autonomy thwarting rather than autonomy supportive reliably increased the odds of a high aggressive trajectory.

In fact, some research within SDT suggests that controlling parenting can result not only in poor internalization, but also oppositional motivations. In line with this, parental prohibition of moral misdeeds perceived to be controlling was found to predict increased oppositional defiance (Vansteenkiste, Soenens, et al., 2014). Other studies have established the explanatory role of need frustration in the relation between controlling parenting and oppositional defiance and associated problem behaviors (Van Petegem, Beyers, Vansteenkiste, \& Soenens, in press). Indeed, it seems that much antisocial behavior can be understood as a reaction to controlling, power-assertive strategies in contexts where attachment security and relatedness is low (Kim, Kochanska, Boldt, \& Nordling, 2014).

Ryan, Sheldon, Kasser, and Deci (1996) argued that, to the extent that individuals lack autonomy support and caring involvement (and therefore are deficient in the development of self), they often turn toward extrinsic values to gain and sustain some minimal sense of power, narcissistic importance, and worth, and a corresponding lack of concern with doing for others or contributing to society. In line with this, Kasser et al. (1995), analyzing data from a long term longitudinal sample, found that adolescents who were more materialistic came from homes where both they and their mothers reported that there was less autonomy support, warmth, and security. They also found that more impoverished, high-crime neighborhoods, in confluence with these more controlling and hostile parenting environments, were likely to promote children's placing strong importance on these extrinsic values. Most relevant in this context, Kasser et al.'s analysis of clinical interviews with 
participants in an at-risk population (defined in terms of maternal psychopathology and low socioeconomic status) revealed that those with greater centrality of materialistic values were more likely to be clinically diagnosed as conduct disordered. One important aspect of this work was its clarification that environments that fail to support autonomy and relatedness promote a compensatory emphasis on alienated or substitute needs, such as material acquisitions and visible trappings of worth. These, in turn, activate the so-called antisocial personality, albeit in ways often seen as self-centered. The research also highlights the continuity of motivational dynamics between nonclinical and clinical populations, as caregiving factors associated with materialism, in more extreme forms are associated with conduct disorders. This general pattern of findings is also consistent with Coie and Jacob's (1993) analysis of conduct disorders.

From the perspective of SDT, the development of conduct disorders and a self-focused goal orientation both stem directly from inadequate attachment and failed internalization resulting from an externally controlling, inconsistent, and affection-impoverished social context as they interact with vulnerabilities to poor self-regulation. To the extent that social values (and the economic conditions that structure them) disable, distract, or fragment the caretaking environment, then children will be more oriented to narcissistic goals to gain a temporary sense of worth and importance. This model is applicable not only to conduct-disordered children but increasingly also to American culture more generally (Kasser, 2002; Twenge et al., 2010). Put succinctly, the more we create conditions that disrupt the quality and stability of familial relationships, the more narcissistically oriented and antisocial our culture as a whole may become.

In sum, conditions of need thwarting, particularly threats to and deprivations of autonomy and relatedness satisfactions in childhood conduce to lowered attachment and interpersonal connectedness, to poorer development of self-regulation and thus impoverished internalization, and to need substitutes in the form of hedonic and materialistic aims, rather than prosocial ones. While such conditions negatively affect wellness in general (e.g., Kasser et al., 1995) when applied to vulnerable individuals the result is often more entrenched antisocial patterns of behavior.

\section{Severe Need Thwarting in Dissociative Identity and Borderline Personality Disorders}

We have argued that essential to the formation of integrated experience and behavioral regulation is support for autonomy, competence, and relatedness. Dissociative identity disorders (DID) and borderline personality disorders (BPD) represent, in most cases, disorders befalling individuals whose life experiences were characterized by not only deprivation, but also active intrusive thwarting, of both autonomy and relatedness needs. In fact, they represent disorders where there has been a failure in the average expectable environments needed for psychological development. For example, Johnson et al. (2006), using a longitudinal sample, reported that low affection (low relatedness) and punitive (low autonomy support) parenting practices were associated with elevated risk for borderline and other personality disorders. Steinberg and Schnall (2001) reported extremely high rates of early physical and sexual abuse among patients with DID. In the histories of people with either disorder, important others who should have been loving and kind to them were instead hurtful and maltreating, violating of the minds and bodies of the people who needed their support. The compensatory results of this are less integrated functioning and diminished sense of autonomy and connection.

\section{Borderline Personality Disorder}

Borderline personality disorders have become a predominant concern in clinical settings both because of their increasing incidence and thus the personal resources demanded in their treatment. Studies of BPD highlight many issues in character pathology generally, and they represent a prototypic example of structural damage to the self that has been associated with caregiver failures to support autonomy and relatedness in early development (Ryan, 2005).

The core feature of BPD is the lack of a cohesive and stable sense of self. Associated with this lack of a consistent and organized self are highly labile emotions, interpersonal relations, and self-esteem dynamics. Borderline individuals show the externalizing attributes of impulsivity, along with some of the features of internalizing disorders such as susceptibility to depression, anxiety, and fragmentation in the face of self-esteem related losses. A cardinal feature of BPD is anger, both self- and other-directed, which can result in destructive actions and magnify relationship volatility and internal feelings of being overwhelmed and disintegrated. More generally, patients with BPD have difficulty differentiating internal needs from external reality, and they are tremendously dependent on concrete supports from others to maintain a sense of self. They lack the internal regulatory processes to modulate anxiety, which can escalate to panic proportions, particularly when no one is available to contain and comfort them. 
Persons with BPD also often lack a stable identity or sense of purpose (Meissner, 1988). Patients with this disorder may, however, latch on to something or someone in an effort to derive a temporary feeling of cohesion, but these choices are often inappropriate or destructive. Commitments are difficult because borderline individuals lack a stable and cohesive self that can form the basis for sharing in committed relationships or endeavors. Connected with the lack of feeling of identity, patients with BPD may feel empty and isolated (Westen, 1991). Clearly, there is a diminution of their true self whereby they lose connection to their interests and feelings. Patients with this disorder often report feelings of boredom and may engage in impulsive acts, such as substance abuse, careless spending, and binge eating, to counteract such feelings. Closely related to this is borderline individuals' lack of capacities for reflective awareness, especially when inner urges are strong (Bleiberg, 2004; Ryan, 2005).

Phenomenologically, patients with BPD often report being both controlled and helpless with respect to their behavior. They often feel like a victim of circumstances without a sense of responsibility for the direction of their own fate. One late adolescent patient, for example, reported that prior to self-mutilation he entered into a lost state, where the overwhelming impulse to cut came on him (impersonal perceived locus of causality), while at the same time he felt he could obtain relief and release only from dysphoric self-hate by engaging in such acts (suggesting external causality). In no sense did he feel autonomous and volitional in these acts, but rather driven, desperate, and helpless.

Although there is some evidence of genetic contributions to BPD, in that many appear to have exhibited a difficult child temperamental profile, much of the etiology appears to be associated with care-giving environments (e.g., Silk, Lee, \& Hill, 1995). As we discussed in attachment research, formation of a stable and cohesive sense of self depends on nurturance in the form of autonomy support and positive involvement. Yet as Sperry (2003) noted, those with BPD have typically experienced severely impoverished care-giving during these early years, with parents' (particularly mothers) having difficulty allowing the children to move toward self-sufficiency and autonomy. Through being both controlling and inconsistent, parents of individuals with BPD fail to bolster the children's integrated self that is essential for the tasks of emotion regulation and identity formation. Having been exposed to caregivers who did not provide autonomy support and were unable to take the children's perspectives, the children failed to develop the sense of reflective awareness that would allow them to be in touch with their own internal states or the internal states of others.

Early caregivers of individuals with borderline disorder have often been described as unavailable, inconsistent, and neglectful, with frequent reports of sexual, emotional, and physical abuse (Sperry, 2003; Zanarini, 1997). In one study, $80 \%$ of borderline patients were reported to be physically or sexually abused or to have witnessed serious domestic violence (Herman et al., 1989). Explicating more specifically the affect and behavior regulation difficulties of these patients, Linehan (1993) suggested that patients with borderline disorder often come from families that invalidate the affective experience of their children. There is little tolerance for fears or anxieties in their children, and they do not offer soothing or comfort when needed. Without such care, these children have difficulty internalizing the capacity to self-soothe, resulting in emotion regulation difficulties, especially being unable to tolerate feelings of distress.

According to object relations theorists (Masterson, 1985), the disorder has its roots in the mother-child relationship, particularly during the phase of separationindividuation when the child is striving to experience him or herself as separate from the mother. Part of the phase involves a pushing away from the mother. In families of borderline individuals, the mothers are not able to tolerate movement toward self-sufficiency as it brings up their own fears of abandonment. Consequently, the mothers threaten to withdraw nurturance from the children if they move to act as separate autonomous individuals. Given this level of conflict, there is no "good" object that can support the psychological needs underlying a cohesive sense of self.

The problems of borderline patients thus illustrate how lack of empathy and consistent involvement and autonomy support undermine intrinsic interests and tendencies and self-regulatory functions - all aspects of a healthy autonomous functioning (Ryan, 2005). These developmental thwarts are thus critical to the formation of BPD Sadly patients with BPD thus have ongoing experiences of lack of need satisfaction, as they have difficulty trusting in relationships and often find themselves acting without autonomy, both contributing to inner distress and continuing cycles of dysregualtion.

\section{Dissociative Identity Disorder}

Dissociative disorders are a particularly important issue within SDT given the theory's focus on autonomy as integration and synthesis. That is, SDT defines autonomy as the integrated regulation of behavior, and dissociative disorders, by definition represent the absence of such integrated regulation. There are two broad senses in which 
dissociative disorders can represent nonintegration. Some dissociative disorders concern a lack of integration between behavior and experience with a sense of self. This is evident in depersonalization symptoms and when there is amnesic or accessibility barrier for certain event or actions. More extreme is the issue of multiplicity, the idea of more than one center of consciousness, expressing distinct motivations, memories, and attributes.

Dissociative disorders thus fundamentally represent a substantial breakdown in self-integration (Ryan et al., 2006). Related by some to an extreme form of BPD, as well as to severe PTSD, most clinicians and researchers also view most dissociative disorders, and particularly dissociative identity disorder (DID) as frequently a product of severe maltreatment or loss (although see Lynn, Lilienfeld, Merckelbach, Giesbrecht, \& van der Kloet, (2012) for a contrary perspective.) Dissociation represents a protective or defensive mechanism, originally arising to protect integrity of a self that is beset with trauma. However, when inability to integrate experiences is chronic or sever, fragmentation of self-functioning and increased tendency to use dissociation to cope with threat occur (Carlson, Yates, \& Sroufe, 2011).

DID manifests as compartmentalized functioning, in which traumatic experiences are housed in dissociated, often inharmonious, parts of personality. Yet more distinctly, DID symptoms include alterations between distinct identity states, often accompanied by amnesic barriers between them. Given its traumatic origins, symptoms of DID overlap with those of both BPD post-traumatic stress disorder. Yet DID is also commonly related to depression, associated with both developmental deficits and helplessness within the condition. In part this may be relate to a common pattern in which, in an attempt to maintain relatedness to an abusing or harsh caregiver, the child internalizes self-critical and self-blaming views (e.g., Westen, 1991). Low self-esteem and depressive affect thus commonly accompany DID and other dissociative disorders.

DID is an autonomy disturbance insofar as autonomy requires cohesiveness and integration, and the violation of self, especially by caregivers, can shatter integrity. The splitting of personality has the function of defensively shielding the core self from pain or harm (van der Hart, Nijenhuis, $\&$ Steele, 2006), but the shifts in identity that characterize DID are typically not experienced as within personal control. Indeed, they have an impersonal perceived locus of causality (Ryan et al., 2006). Depersonalization, another symptom associated with DID, also exemplifies this sense of impersonal causality, as if one's body were acting independently of one's will. As autonomy, or self-rule, depends on a sense of self and an internal perceived locus of causality for behavior, it is clear that autonomy is seriously disturbed when behavior occurs without choice or in a dissociated state.

The perceived lack of personal causation is often part of the despondency and depression these patients report. For example, a patient who came to treatment for depression and for concerns for her memory for events had manifest symptoms of DID. She subsequently revealed repeated sexual abuse by her father that she previously had not reported. Images began to surface, often uncontrollably. As memories unfolded it was also clear that she had been repeatedly threatened if she ever told anyone, a command she had internalized. Various parts of her story, and emotional reactions to it, were represented by different personality systems, each with its own age and makeup, and its own capacity to exert executive control under particular circumstances.

As van der Hart and colleagues (2006) pointed out, whereas healthy personality functioning is characterized by synthetic, coordinated functioning, the defensively precipitated identities in DID are often very simple and focused, and not prone toward integration or harmony with other personality subsystems. Van der Hart et al.'s treatment approach focuses on increasing connectivity between split off parts of personality, using a slow process of exposure and dialog in which compassion and understanding are fostered, along with encouragement to move toward feared or avoided opportunities for self-realization, to further promote such connectivity. Clinicians who have worked with DID know the difficulty of the process approach and the required patience and empathy. Often change requires strong supports and anxiety reduction strategies as well. Adaptation and tools for grounded self-regulation rather than full integration is often an appropriate goal. The goal of course is the recovery more coherent, integrated functioning, and the resumption of self-development to whatever extent possible.

In sum, a fundamental tenet of developmental psychopathology is that the study of both normal and atypical development should inform each other (Cicchetti, 2006; Cicchettti \& Toth, 2009). SDT's research into basic psychological needs richly links psychologies of growth with those of pathology. Evidence shows how the psychological nutrients that facilitate optimal development, when neglected or thwarted can have cascading negative effects across development. Moreover, the kind of need thwarting a child experiences can be related to the kind of pathology he or she manifests. We identified three types of disorders reflecting different types of autonomy disturbance: 
internalizing disorders, impairments in internalization, and self-fragmentation. Each reflects a varied way the integrated self- regulation and ownership of actions is disrupted, and each also has autonomy thwarting as a featured symptomatic or etiological factor.

\section{Translational Implications: Autonomy and Autonomy Support in Psychotherapy and Intervention Programs}

An interesting question concerns the relations between etiological factors such as lack of autonomy, relatedness, and competence supports in development to ameliorative factors proposed by various treatment approaches to psychological problems. It seems clear that the absence of need-supportive inputs from caregivers can catalyze an array of negative developmental processes, both behavioral and biological, that eventuate in disordered functioning. Reciprocally, in efforts to treat the immediate harms and cascading negative effects of such social etiological factors, SDT suggests that need supportive treatment and intervention contexts are critical.

Ameliorating or treating existing disorders often entails addressing need deficits both directly, by providing a need supportive therapeutic context, and indirectly, for example by providing people with skills or opportunities to better satisfy needs in their natural environments. In other words, SDT suggests therapy is, in large part, effective through facilitating the individuals' capacities to act with autonomy, and in acting so, to satisfy all three basic psychological needs (Ryan \& Deci, 2008).

Autonomy support is of course important to all schools of psychotherapy, especially because volitional motivation is so necessary to therapeutic engagement and success. For example, Ryan, Lynch, Vansteenkiste, and Deci (2011), reviewing approaches to psychotherapy, and behavior change distinguished between outcome and process focused therapies. Outcome focused approaches (e.g., behavioral and cognitive behavioral treatments) tend to promote autonomy through an emphasis on transparency. Patients are told beforehand what is expected and allowed to select in to treatment, or not. Readiness for treatmen can also be facilitated through motivational enhancement therapies, such as brief motivational interviewing (MI; Miller \& Rollnick, 2002) as adjuncts to outcome-focused interventions to prepare the individual to volitionally engage the therapy. Ryan et al. pointed out that more process-oriented schools of therapy (e.g., psychodynamic and existential approaches) conceive of motivation as a focus of treatment itself rather than something that facilitates pre-treatment readiness. Cultivating autonomous engagement and taking interest in resistances is in fact considered an active and ongoing focus of process-oriented therapies.

Zuroff et al. (2007), drawing on SDT, proposed that autonomy should be considered a common factor in all effective psychological treatments. Supporting their proposal, they reported a study of depressed outpatients who were randomly assigned to receive interpersonal therapy, cognitive-behavioral therapy, or pharmacotherapy with clinical management. Assessments of depression severity were taken before and after treatment, and therapeutic alliance, the clients' autonomous motivation, and therapists' autonomy support were measured at the third session within each group. Zuroff et al. found first, as expected by SDT that therapist autonomy support was associated with greater client autonomous motivation. More strikingly, autonomous motivation was a better predictor of improved outcome than therapeutic alliance across all three treatments. The researchers therefore suggested that the promotion of autonomy is an important factor in treatment across modalities and can be distinguished from therapeutic alliance per se.

Given its focus on facilitating autonomous motivation, not surprisingly SDT has increasingly been used as a guiding framework for clinical interventions and randomized clinical trials in behavior change (Ryan, Patrick, Deci, \& Williams, 2008). In fact clinical studies applying SDT attest to the positive consequences of autonomy-supportive treatment climates, including higher treatment attendance, less dropout, less relapse, and enhanced well-being over the course of treatment. Such results have been obtained in various domains such as drug and alcohol dependence (Foote et al., 1999; Ryan, Plant, \& O’Malley, 1995; Zeldman, Ryan, \& Fiscella, 2004), weight loss (Williams, Grow, Freedman, Ryan, \& Deci, 1996), smoking cessation (Williams, McGregor, et al., 2006), general medication adherence (Williams, Rodin, Ryan, Grolnick, \& Deci, 1998), HIV-related medication adherence (Kennedy, Gogin, \& Nollen, 2004), eating disorders (Van der Kaap-Deeder et al., 2014), and other issues.

Beyond the importance of autonomy for behavior change is its importance in restorative self-development and personality growth. These principles are critical both in psychotherapy and in programs that provide support and training for caregivers. Particularly germane to the issue of motivation in growth-focused settings is a focus on autonomy support, which conduces exploration, disclosure, and integration (Weinstein, Przybylski, \& Ryan, 2012). Specific behaviors associated with autonomy support include (1) taking the child's or adolescent's internal frame 
of reference and welcoming negative feelings and resistance; (2) providing a meaningful rationale for requests and activities; (3) minimizing external controls such as contingent rewards and punishments or conditional regard, instead nurturing inner motivational resources (e.g., challenge, curiosity); (4) providing opportunities for participation, input, and choice; and (5) following the child's rhythm of progress and development (Reeve, 2009; Ryan \& Deci, 2008). In autonomy-supportive contexts, pressure to engage in specific behaviors or express specific attitudes is minimized, and individuals are encouraged to base their actions on their own reflective considerations and values. Thus, autonomy for behavior is facilitated insofar as actors are helped to identify and own their reasons for changing or not changing behaviors, and do not feel pressured toward specific outcomes. This in turn leads to greater internalization and maintenance of change.

In addition to experiencing autonomy, internalization also requires that a person experience the confidence and competence to change. In SDT, competence support is afforded when practitioners provide the effectance-relevant inputs of structure and feedback (Jang, Reeve, \& Deci, 2010; Sierens, Vansteenkiste, Goossens, Soenens, \& Dochy, 2009). Clients are afforded the skills and tools for change and are empathically supported when efficacy-related barriers emerge. In the SDT model of change, gaining a sense of competence is facilitated by autonomy, in that, once people are volitionally engaged and have a high degree of willingness to act, they are then most apt to learn and apply new strategies and competencies (Markland et al., 2005). At that point providing additional challenges and structure can be especially helpful.

Finally SDT considers relatedness support as critical in both growth and internalization processes. Unconditional positive regard and helpful, but not intrusive, involvement facilitate relatedness experiences, which support interest and curiosity, as well as the trust and connectedness important to internalization. However, unconditional regard is not merely a technique, and it must be perceived as authentic or genuine to have the functional significance of relational support (Ryan, 1995)

These general principles can be actively applied in programs to train teachers (e.g., Aelterman, et al., in press; Cheon, Reeve, \& Moon, 2012) or parents to adopt a more need-supportive approach, as to enhance developmental outcomes and lessen psychopathology. For example, Joussemet, Mageau, and Koestner (2013) studied an intervention program called the How-to Parenting Program, which included components meant to enhance provision of structure, greater relatedness and more support for autonomy. Results indicated the program succeeded in increasing these parenting attributes relative to baseline, and moreover, the level of children's internalizing and externalizing symptoms were significantly decreased, suggesting program effectiveness.

Future translational efforts must also move beyond tertiary efforts at psychotherapy and behavior change, toward more prevention, and this is a goal for which SDT's body of knowledge is uniquely suited. SDT, as an empirically supported framework, points both to general facilitating factors (e.g., psychological need satisfactions), and criteria for testing any specific strategies for enhancing these factors (i.e., measurements of targeted motivational mediating variables). There is thus great promise ahead for both intervention and process studies of behavior change and for creating conditions in families, schools, and communities that build resilience through fostering capacities for autonomy.

\section{SDT AND THE STUDY OF DEVELOPMENT AND PSYCHOPATHOLOGY: CONCLUSIONS AND IMPLICATIONS}

Among the central aims of SDT is the explication of the social-contextual conditions that promote optimal development of self. Thus, we have focused considerable attention on specifying the conditions that both facilitate and undermine optimal development. In this way, basic psychological needs are constructs that link SDT's efforts in understanding growth and positive experience, to the study of ill-being and the development of psychopathology. It is our contention that many of the processes that are integral to nonoptimal functioning in normal populations are also central to various psychopathologies. Indeed, we see continuity in the basic psychological needs for autonomy, competence, and relatedness across people's personality differences, developmental epochs, and cultures, suggesting that thwarting of these basic needs, especially when being severe, is a critical component of psychopathology and more normal or transitory maladjustment.

The developmental antecedents of disturbed autonomy are manifold, with genetic, biological, interpersonal, and sociocultural factors all being relevant (Cicchetti \& Dawson, 2002; Ryan \& Hawley, in press). Genetic and other biological factors enter transactionally into interpersonal relationships, facilitating or forestalling the quality of these social contextual inputs and thus constituting an obstacle or affordance for experienced need satisfaction, and cultural factors both shape and are emergent from patterns of social and familial functioning. Our focus has 
been primarily on the social and familial factors, although our aim was not to provide a complete account of the development of autonomy disturbances. Rather, we have attempted to describe the phenomenological significance of autonomy in normal and pathological development and to show empirically and theoretically how interpersonal factors contribute to the etiology of pathologically disturbed autonomy.

Within this approach, we have viewed the developmen of autonomy as proceeding most effectively in familial and social contexts that provide autonomy support, optimal structure, and interpersonal involvement. In the absence of these necessary social nutrients - in contexts that thwart satisfaction of the needs for autonomy, competence, and relatedness-disturbed self-development is expected, resulting in the emergence of psychopathology. Psychopathology is thus the result of disorganizing influences, of contexts that thwart or forestall personality integration.

We have reviewed a large number of studies indicating that the development of autonomy - that is, the maintenance of intrinsic motivation, the internalization of values and regulatory processes, the integration of emotions, and the formation of identities - is facilitated by the contextual nutrients of caregiver attention and interest, and of encouragement for exploration and self-initiation. Contexts where interpersonal involvement and autonomy support are absent have been found reliably to diminish autonomous regulation and impair the development of self. The contextual elements that have consistently been found in our studies to impair autonomy and development - namely, controlling or uninvolved parenting-have also been emphasized in the clinical literature on the antecedents of disorders that involve either heteronomous introjects or failures of internalization. Thus, there appear to be clear parallels between the results of the empirical explorations of autonomy dynamics in normative development and the conclusions from clinical studies of psychopathology. Indeed, basic psychological needs represent constructs linking our understanding of both growth and pathology in self-development (Vansteenkiste \& Ryan, 2013)

\section{Future Directions}

SDT focuses on the role of basic psychological needs in both growth and pathology. What is exciting about the convergence between SDT-grounded research results on both healthy populations and those experiencing psychopathology is that it sets the stage for more integrative empirical investigations in which the concepts of autonomy, competence, and relatedness needs figure heavily. For example, the constructs concerning intrinsic motivation, internalization, and autonomy-supportive relationships all bear on clinical phenomena, and they help us see the continuity of human needs in diverse populations. Whereas theories of internalization have been widely discussed in developmental and clinical literatures by such seminal writers as Freud, Mahler, Erikson, Perry, Piaget, and Kohlberg to name a few, there has been a paucity of empirically-based studies of the process, and little specific theory about factors that facilitate versus undermine internalization in clinical settings. SDT's empirical focus provides testable hypotheses, amenable to both experimental and field methods, concerning both how to measure internalization, and its antecedents, consequences, and neuropsychological correlates.

More detailed work on the antecedents of specific autonomy disturbances within the various disorders will also be important. It is clear, for example, that the lack of autonomy support and genuine relatedness by caregivers are antecedent to the development of a wide range of pathologies (Ryan et al., 2006). Yet why an individual develops anorexia nervosa rather than pervasive self-critical depression in familial contexts that are demanding and critical is an example of the kinds of questions that are important to tackle empirically, and in coordination with epigenetic and neuroscience findings. Although failures of autonomy support appear to supply a generalized stressor to integrated development, how this differentially impacts and interacts with varied diatheses and vulnerabilities is an area for further inquiry. We know far too little about the specific pathways to specific disorders, leaving the study of factors that conduce to or elicit particular pathologies an important agenda for continued research.

The theoretical framework of SDT specifically distinguishes autonomy from independence, and this distinction is one we see as particularly critical to the field of developmental psychopathology, as well as to theories of parenting, attachment, and development more generally Noting that both independence and dependence can be either heteronomous or autonomous can help future researchers disentangle what heretofore has been a very mixed and confused literature on dependence across the life span. In our view, dependence should be viewed not only as a potential problem but also as a positive capacity. The willingness to rely on and receive support from others is a basic human propensity that is fostered when autonomy is supported (Ryan et al., 2005). Future research can thus build on this distinction, examining more clearly the pros and cons of dependencies in different developmental epochs and interpersonal contexts. It can also inform 
the growing literatures on psychopathology as it relates to gender and culture, where issues of autonomy and independence have too often been melded and confused (Chirkov, Ryan, \& Sheldon, 2011).

More generally, we have pointed to the connections between the development and integration of personality and the phenomenological experience of autonomy in the regulation of behavior. In our view, the issue of autonomy is a critical one for organizational perspectives on developmental psychopathology. The experience of autonomy is a defining feature of organization, whereas disturbances of autonomy that correspond to fragmentation and disorganization in psychological development are also manifest as experiences of control and amotivation. We have further pointed to environmental conditions that either thwart or nurture needs for autonomy, competence, and relatedness as influential factors in development either away from or towards greater organization and integrity, respectively. The differentiated study of how these psychological needs, in interaction with the biological and social conditions of development, result in relative integration and healthy self-regulation supplies a broad and important agenda for future clinical research, and for the development of a knowledge base applicable in clinical interventions.

Because of their fundamental importance in psychological development and their pervasive expression in human behaviors, there is clearly more to know about the nature of basic psychological needs, their evolution, and their mechanistic underpinnings. Moreover, research is only nascent regarding their dynamic interaction with physiological or physical needs (Chen, Van Assche, Vansteenkiste, Beyers, $\&$ Soenens, in press). Finally, there is much more research needed about micro- and macrosocial contextual factors impacting their satisfaction or frustration. Progress is rapidly being made on the neuropsychological underpinnings of autonomous and controlled forms of motivation, as we have reviewed. However, much of this knowledge is still at the descriptive level and has yet to be coordinated with genetic studies, and the differential expression of human capacities for growth and defense over development. For example, we know that need thwarting impacts more than immediate experience and behavior and can have cascading effects in multiple areas, from social adjustment to executive functioning (e.g., Bernier, Carlson, \& Whipple, 2010; Vansteenkiste \& Ryan, 2013). Understanding how need frustrations have these arrayed effects is a matter that will require both more refined empirical and theoretical efforts at every level of analysis.

Developmental psychology, in fact, offers windows into our evolved tendencies and their selective advantages
(Ryan \& Hawley, in press; Tomasello, 2008). The fact that basic psychological needs universally play a role in healthy psychological development and wellness suggests that further studies, both genetic and comparative, will add to our understanding of their role in adaptation and maladaptation. More generally, SDT has grown very rapidly in recent years, and its body of evidence contains studies of persons in every part of the life span. Yet there needs to be much deeper understanding of both how need satisfactions change over time in character and focus and how they support developmental progressions in multiple developmental lines.

As a universalistic, organismic perspective on development and personality functioning SDT is also inherently a cross-cultural psychology. Cultures, by their behavioral norms, environmental affordances, and familial practices differentially impact need satisfactions and frustrations. Although the cross-cultural research in SDT has largely focused to date on showing commonalities in effects of satisfaction and frustration of basic needs across groups, domains and cultures, as a maturing scientific perspective it is also time for SDT to take closer interest in the differences these contexts set in motion. For instance, there may exist some cross-cultural variability in the way the psychological needs get nurtured and satisfied (Soenens, Vansteenkiste, \& Van Petegem, in press). Along with this, greater attention is needed on cultural differences that enhance or undermine the adopted and internalization of need supportive tertiary and preventive interventions.

Beyond culture we also need more empirical research on how conditions of impoverishment and oppression at cultural levels impact developmental outcomes as mediated by need supports and thwarts. Both proximal and global conditions affect human psychological need satisfactions, so just as we need to coordinate the study of psychological needs with their mechanistic underpinnings, understanding the more global social conditions that affect human wellness is critical to research that can inform policies on child welfare, education, and the necessary social supports to facilitate optimal development for children of all social backgrounds. Illustratively, two recent studies demonstrate how differences in socioeconomic status and the presence of income inequalities negatively affect people's physical and mental health (Di Domenico \& Fournier, 2014; Gonzales, Swanson, Lynch, \& Williams, 2014). Insofar as conditions of poverty and deprivation conduce to psychopathology through the frustration of basic needs, global efforts to address such conditions and to establish greater social justice deserve our attention both as clinical scientists and as world citizens. 


\section{REFERENCES}

Abuhamdeh, S., \& Csikszentmihalyi, M. (2012). The importance of challenge for the enjoyment of intrinsically motivated, goal-directed activities. Personality and Social Psychology Bulletin, 38, 317-330 doi 10.1177/0146167211427147

Aelterman, N., Vansteenkiste, M., Van den Berghe, L., De Meyer, J. \& Haerens, L. (in press). Fostering a need-supportive teaching style: Intervention effects on physical education teachers' beliefs and teaching behaviors. Journal of Sport \& Exercise Psychology.

Ahmad, I., Vansteenkiste, M., \& Soenens, B. (2013). The relations of Arab Jordanian adolescents' perceived maternal parenting to teacher-rated adjustment and problems: The intervening role of perceived need satisfaction. Developmental Psychology, 49, 177-183.

Ainsworth, M. D. S., Blehar, M. C, Waters, E., \& Wall, S. (1978). Pattern of attachment. Hillsdale, NJ: Erlbaum.

Amabile, T. M. (1983). The social psychology of creativity. New York, NY Springer- Verlag.

Aunola, K., \& Nurmi, J. E. (2005). The role of parenting styles in children's problem behavior. Child Development, 76, 1144-1159.

Appiah, A. K. (2005). The ethics of identity. Princeton, NJ: Princeton University Press.

Assor, A. (2012). Allowing choice and nurturing an inner compass: Educational practices supporting students' need for autonomy. In S. L. Christenson, A. L. Reschly \& C. Wylie (Eds.), Handbook of research on student engagement (pp. 421-439). New York, NY: Springer.

Assor, A., Roth, G., \& Deci, E. L. (2004). The emotional costs of parents' conditional regard: A self-determination theory analysis. Journal of Personality, 72, 47-88. doi: 10.1111/j.0022-3506.2004.00256.x

Assor, A., Vansteenkiste, M., \& Kaplan, A. (2009). Identified versus introjected approach and introjected avoidance motivations in school an in sports: The limited benefits of self-worth strivings. Journal of Educational Psychology, 101, 482-497. doi: 10.1037/a0014236

Assor, A., \& Tal, K. (2012). When parents' affection depends on child's achievement: Parental conditional positive regard, selfaggrandizement, shame and coping in adolescents. Journal of Adolescence, 35, 249-260. doi: 10.1016/j.adolescence.2011.10.004

Baldwin, A. L (1955). Behavior and development in childhood. New York, NY: Dreyden

Bandura, A. (1989). Human agency in social cognitive theory. American Psychologist, 44, 1175-1184. doi: 10.1037/0003-066X.44.9.1175

Barber, B. K. (1996). Parental psychological control: Revisiting neglected construct. Child Development, 67, 3296-3319. doi: 10.1111/ j.1467-8624.1996.tb01915.x

Barber, B. K., Stolz, H. E., \& Olsen, J. A. (2005). Parental support, psychological control, and behavioral control: Assessing relevance acros time, method, and culture. Monographs of the Society for Research in Child Development, 70(4, Serial No. 281)

Baumeister, R., \& Leary, M. R. (1995). The need to belong: Desire for interpersonal attachments as a fundamental human motivation. $P$ sychological Bulletin, 117, 497-529. doi: 10.1037/0033-2909.117.3.497

Baumrind, D. (1996). The discipline controversy revisited. Family Rela tions, 45, 405-414. doi: $10.2307 / 585170$

Baumrind, D. (2012). Differentiating between confrontive and coercive kinds of parental power-assertive disciplinary practices. Human Development, 55, 35-51. doi: 10.1159/000337962

Bayer, J. K., Sanson, A. V., \& Hemphill, S. A. (2006). Parent influences on early childhood internalizing difficulties. Journal of Applied Developmental Psychology, 27 (6), 542-559. doi: 10.1016/j.appdev. 2006.08.002
Bechara, A. Tranel, D. Damasio, H., \& Damasio, A. R. (1996). Failure to respond autonomically to anticipated future outcomes following damage to prefrontal cortex. Cerebral Cortex, 6, 215-225 doi: 10.1093/ cercor/6.2.215

Beck, A. T. (1983). Cognitive treatment of depression: New perspectives. In P. J. Clayton \& J. E. Barrett (Eds.), Treatment of depression: Old controversies and new approaches (pp. 265-290). New York, NY: Raven Press.

Belsky, J. (2002). Developmental origins of attachment styles. Attachment and Human Development, 4 (2), 166-170.

Benjamin, L. S. (2003). Interpersonal diagnosis and treatment of personality disorders. New York, NY: Guilford.

Bernier, A., Carlson, S. M., \& Whipple, N. (2010). From external regulation to self-regulation: Early parenting precursors of young children's executive functioning. Child Development, 81, 326-339.

Blais, M. R., Sabourin S., Boucher, C, \& Vallerand, R. J. (1990). Toward a motivational model of couple happiness. Journal of Personality and Social Psychology, 59, 1021-1031. doi: 10.1037/00223514.59.5.1021

Blatt, S. J. (1974). Levels of object representation in anaclitic and introjective depression. Psychoanalytic Study of the Child, 29, 107-157.

Bleiberg, E. (2004). Treating personality disorders in children and adolescents: A relational approach. New York, NY: Guilford.

Blos, P. (1979). The adolescent passage. New York, NY: International Universities Press.

Bober, S., \& Grolnick, W. S. (1995). Motivational factors related to differences in self-schemas. Motivation \& Emotion,19, 307-327. doi 10.1007/BF02856517

Boone, L., Vansteenkiste, M., Soenens, B., Van der kaap-Deeder, J., \& Verstuy f, J. ( 2014). Self-critical perfectionism and binge eating symptoms: A longitudinal test of the intervening role of psychological need frustration. Journal of Counseling Psychology, 61, 363-373. doi: $10.1037 / \mathrm{a} 0036418$

Bouchard, G., Lee, C. M., Asgary, V., \& Pelletier, L. G. (2007). Fathers' motivation for involvement with their children: A self-determination theory perspective. Fathering, 5, 25-41.

Bowlby, J. (1969). Attachment and loss: Vol. 1: Attachment. New York, NY: Basic Books.

Bradley, S. J. (2000). Affect regulation and the development of psychopathology. New York, NY: Guilford.

Brenning, K., Soenens, B., Braet, C., \& Bal, S. (2012). The role of parenting and mother-adolescent attachment in the intergenerational similarity of internalizing symptoms. Journal of Youth and Adolescence, 41, 802-816. doi: 10.1007/s10964-011-9740-9

Brenning, K., Soenens, B., Van Petegem, S., \& Vansteenkiste, M. (in press). Perceived maternal autonomy support and early adolescent emotion regulation: A longitudinal study. Social Development.

Bretherton, I. (1987). New perspectives on attachment relations: Security, communication and internal working models. In J. Osofsky (Ed.), Handbook of infant development (pp. 1061-1100). New York, NY: Wiley.

Bruch, H. (1973). Eating disorders. New York, NY: Basic Books.

Bruch, H. (1979). The golden cage: The enigma of anorexia nervosa. New York, NY: Vintage Books.

Brunstein, P. (1994). Patterns of parent-child interaction in Mexican families: A cross-cultural perspective. International Journal of Behavioral Development, 17, 423-446.

Burnette, M. L., \& Cicchetti, D. (2012). Multilevel approaches toward understanding anti-social behavior: Current research and future directions. Development and Psychopathology, 24, 703-704. doi: 10.1017/ S0954579412000314 
Cacioppo, J. T. Berntson, G. G., Sheridan, J. F. \& McClintock, M. K. (2000). Multilevel integrative analyses of human behavior: Social neuroscience and the complementing nature of social and biological approaches. Psychological Bulletin, 126, 829-843. doi: 10.1037/l 0033-2909.126.6.829

Carlson, E. A., Yates, T. M., \& Sroufe, A. L. (2011). Dissociation and the development of self. In P. F. Dell \& J. A O'Neil(Eds.), Dissociation and the dissociative disorders: DSM V and beyond (pp. 39-53). New York, NY: Routledge

Cerasoli, C. P. Nicklin, J. M., \& Ford, M. T. (2014). Intrinsic motivation and extrinsic incentives jointly predict performance: A 40-year meta-analysis. Psychological Bulletin, 140(4), 980-1008. doi: 10.1037/ a0035661

Chambers, R. A., Taylor, J. R., \& Potenza, M. N. (2003). Developmental neurocircuitry of motivation in adolescence: A critical period of addiction vulnerability. American Journal of Psychiatry, 160, 1041-1052. doi: 10.1176/appi.ajp.160.6.1041

Chen, B., Vansteenkiste, M., Beyers, W., Soenens, B., \& Van Petegem, S. (2013). Autonomy in family decision making for Chinese adolescents: Disentangling the dual meaning of autonomy. Journal of Cross-cultural Psychology, 44, 1184-1209. doi: 10.1177/002202211 3480038

Chen, B., Vansteenkiste, M., Beyers, W., Boone, L., Deci, E. L., Duriez, B. .... Verstuyf, J. (in press). Basic psychological need satisfaction, need frustration, and need strength across four cultures. Motivation and Emotion.

Chen, B., Van Assche, J., Vansteenkiste, M., Soenens, B., \& Beyers, W. (in press). Does psychological need satisfaction matter when environmental or financial safety are at risk? Journal of Happiness Studies.

Cheon, S. H., Reeve, J., \& Moon, I. S. (2012). Experimentally based, longitudinally designed, teacher-focused intervention to help physical education teachers be more autonomy-supportive toward their students. Journal of Sport \& Exercise Psychology, 34, 365-396.

Cheung, C. S. S., \& Pomerantz, E. M. (2011). Parents' involvement in children's learning in the United States and China: Implications for children's academic and emotional adjustment. Child Development 82 (3), 932-950. doi: 10.1111/j.1467-8624.2011.01582.x

Chirkov, V., \& Ryan, R. M. (2001). Parent and teacher autonomy-support in Russian and U.S. adolescents: Common effects on well-being and academic motivation. Journal of Cross-Cultural Psychology, 32, 618-635. doi: 10.1177/0022022101032005006

Chirkov, V. I., Ryan, R. M., \& Sheldon, K. M. (Eds.) (2010). Human autonomy in cross-cultural context. New York, NY: Springer.

Chua, S. N., Wong, N., \& Koestner, R. (2014). Autonomy and controlling support are two sides of the same coin. Personality and Individual Differences, 68, 48-52. doi: 10.1016/j.paid.2014.04.008

Cicchetti, D. (2006). Development and psychopathology. In D. Cicchetti \& D. Cohen (Eds.) Developmental psychopathology: Theory and methods (2nd ed., Vol. 1, pp. 1-24). New York, NY: Wiley.

Cicchetti, D., \& Dawson, G. (2002). Editorial: Multiple levels of analysis. Development and Psychopathology, 14, 417-420. doi: 10.1017/S0954 $57940200301 \mathrm{X}$

Cicchetti, D., Ganiban, J., \& Barnett, D. (1991). Contributions from the study of high-risk populations to understanding the development of emotion regulation. In J. Garber \& K. A. Dodge (Eds.), The development of emotion regulation and dysregulation (pp. 15-48). New York, NY: Cambridge University Press.
Cicchetti D., Toth S. L. (2009). The past achievements and future promises of developmental psychopathology: The coming of age of a discipline. Journal of Child Psychology and Psychiatry, 50, 16-25. doi 10.1111/j.1469-7610.2008.01979.

Cicchetti, D., \& Valentino, K. (2006). An ecological transactional perspective on child maltreatment: Failure of the average expectable environment and its influence on child development. In D. Cicchetti \& D. J. Cohen (Eds.), Developmental psychopathology: Vol. 3. Risk, disorder and adaptation (2nd ed., pp. 129-201). New York, NY: Wiley.

Claes, L., Soenens, B., Vansteenkiste, M., \& Vandereycken, W. (2012). The scars of the inner critic: Perfectionism and non-suicidal self-injury in eating disorders. European Eating Disorders Review, 20, 196-202 doi: $10.1002 /$ erv. 1158

Coie, J. D., \& Jacobs, M. R. (1993). The role of social context in the prevention of conduct disorder. Development and Psychopathology, 5 , 263-27. doi: $10.1017 / \mathrm{S} 0954579400004387$

Curran, T., Hill, A. P., \& Niemiec, C. P. (2013). A conditional process model of children's behavioral engagement and behavioral disaffection in sport based on self-determination theory. Journal of Sport \& Exercise Psychology, 35, 30-43

Danner, E W., \& Lonky, E. (1981). A cognitive-developmental approach to the effects of rewards on intrinsic motivation. Child Development 52, 1043-1052. doi: 10.1111/j.1467-8624.1981.tb03147.x

Deci, E. L. (1971). Effects of externally mediated rewards on intrinsic motivation. Journal of Personality and Social Psychology, 18, 105-115. doi: $10.1037 / \mathrm{h} 0030644$

Deci, E. L. (1972). Intrinsic motivation, extrinsic reinforcement, and inequity. Journal of Personality and Social Psychology. 22, 113-120. doi: $10.1037 / \mathrm{h} 0032355$

Deci, E. L., \& Cascio, W. F. (1972, April). Changes in intrinsic motivation as a function of negative feedback and threats. Paper presented at the meeting of the Eastern Psychological Association, Boston, MA

Deci, E. L., Driver, R. E., Hotchkiss, L., Robbins, R. J., \& Wilson, I. M. (1993). The relation of mothers' controlling vocalizations to children's intrinsic motivation. Journal of Experimental Child Psychology, 55, 151-162. doi: 10.1006/jecp.1993.1008

Deci, E. L., Koestner, R., \& Ryan, R. M. (1999). A meta-analytic review of experiments examining the effects of extrinsic rewards on intrinsic motivation. Psychological Bulletin, 125, 627-668 doi: 10.1037/ 0033-2909.125.6.627

Deci, E. L., \& Ryan, R. M. (1985a). The general causality orientation scale: Self-determination in personality. Journal of Research in Personality, 19, 109-134. doi: 10.1016/0092-6566(85)90023-6

Deci, E. L., \& Ryan, R. M. (1985b). Intrinsic motivation and self-determination in human behavior. New York, NY: Plenum.

Deci, E. L., \& Ryan, R. M. (1995). Human autonomy: The basis for true self-esteem. In M. Kernis (Ed.), Efficacy, agency, and self-esteem (pp. 31-49). New York, NY: Plenum.

Deci, E. L., \& Ryan, R. M. (2000). The "what" and "why" of goal pursuits Human needs and the self-determination of behavior. Psychological Inquiry, 11, 227-268. doi: 10.1207/S15327965PLI1104_01

Deci, E. L., \& Ryan, R. M. (2012). Motivation, personality, and development within embedded social contexts: An overview of self-determination theory. In R. M. Ryan (Ed.), The Oxford handbook of motivation (pp. 85-107). New York, NY: Oxford University Press.

Deci, E. L., \& Ryan, R. M. (2013). The importance of autonomy for development and well-being. In B. Sokol, F. M. E. Grouzet, \& U. Muller (Eds.), Self-regulation and autonomy: Social, developmental, educational, and neurological dimensions of human contact (pp. 19-46) New York, NY: Cambridge University Press.

Deci, E. L., Ryan, R. M., Schultz, P. P., \& Niemiec, C. P. (2014). Being aware and functioning fully: Mindfulness and interest-taking within 
self-determination theory. In K. W. Brown, R. M. Ryan, \& J. D. Creswell (Eds.), Handbook of mindfulness. New York, NY: Guilford

Deci, E. L., Schwartz, A. J., Sheinman, L., \& Ryan, R. M. (1981). An instrument to assess adults' orientations toward control versus autonomy with children: Reflections on intrinsic motivation and perceived competence. Journal of Educational Psychology, 73, 642-650. doi: 10.1037//0022-0663.73.5.642

De Charms, R. (1968). Personal causation: The internal affective determinants of behavior. New York, NY: Academic Press.

de Haan, A. D., Soenens, B., Dekovic, M., \& Prinzie, P. (2013) Effects of childhood aggression on parenting during adolescence: The role of parental psychological need satisfaction. Journal of Clinical Child \& Adolescent Psychology, 42, 393-404. doi: 10.1080/ 15374416.2013.769171

De Naeghel, J., Van Keer, H., Vansteenkiste, M., \& Rosseel, Y. (2012) The relation between elementary students' recreational and academic reading motivation, reading frequency, engagement and comprehension: A self-determination theory perspective. Journal of Educational Psychology, 104, 1006-1021 doi: 10.1037/a0027800

Depue, R. A., \& Lenzenweger, M. F. (2001). A neurological dimensional model. In W. J. Livesley (Ed.), Handbook of personality disorders: theory, research, and treatment (pp. 136-176). New York, NY: Guilford.

Di Domenico, S. I., \& Fournier, M. A. (2014). Socioeconomic status, income inequality and health complaints: A basic psychologica needs perspective. Social Indicators Research, DOI 10.1007/s11205 013-0572-8

Di Domenico, S. I., Fournier, M. A., Ayaz, H., \& Ruocco, A. C. (2013). In search of integrative processes: Basic psychological need satisfaction predicts medial prefrontal activation during decisional conflict. Journal of Experimental Psychology: General, 142, 967-978. doi: 10.1037/a0030257

Dittmar, H. (2011). Material and consumer identities. In S. Schwartz, K. Luyckx, \& V. Vignoles (Eds.), Handbook of identity theories and research (p. 745). New York, NY: Springer.

Dworkin, G. (1988). The theory and practice of autonomy. New York, NY: Cambridge.

Eisenberger, R., \& Cameron, J (1996). Detrimental effects of reward: Reality of myth? American Psychologist, 51,1153-1166. doi: 10.1037/ 0003-066X.51.11.1153

Elkind, D. (1967). Egocentrism in adolescence. Child Development, 38 1025-1034. doi: 10.1111/j.1467-8624.1967.tb04378.x

Enns, M. W., Cox, B. J., \& Clara, I. (2002). Adaptive and maladaptive perfectionism: Developmental origins and association with depression proneness. Personality \& Individual Differences, 33, 921-935. doi 10.1016/S0191-8869(01)00202-1

Erikson, E. H. (1950). Childhood and society. New York, NY: Norton.

Farkas, M. S., \& Grolnick, W. S. (2010). Examining the components and concomitants of parental structure in the academic domain. Motivation and Emotion, 34, 266-279. doi: 10.1007/s11031-010-9176-7

Flavell, J. H., Miller, P. H., \& Miller, S. A. (2002). Cognitive development. Englewood Cliffs, NJ: Prentice Hall.

Flett, G. L., Hewitt, P. L., \& Singer, A. (1995). Perfectionism and parental authority styles. Individual Psychology. Journal of Adlerian Theory, Research \& Practice, 51, 50-60.

Foote, J., DeLuca, A., Magura, S., Warner, A., Grand, A., Rosenblum, A., \& Stahl, S. (1999). A group motivational treatment for chemical dependency. Journal of Substance Abuse Treatment, 17, 181-192. doi 10.1016/S0740-5472(99)00003-3

Freud, A. (1966). The ego and the mechanisms of defense. New Your, NY: International Universities Press.
Friedman, M. (2003). Autonomy, gender, politics. New York, NY: Oxford University Press.

Frodi, A., Bridges, L., \& Grolnick, W. S. (1985). Correlates of mastery-related behavior: A short term longitudinal study of infants in their second year. Child Development, 56, 1291-1298. DOI: 10.2307। 1130244

Frost, R. O., Novara, C., \& Rheaume, J. (2002). Perfectionism in obsessive compulsive disorder. In R. O. Frost \& G. Steketee (Eds.), Cognitive approaches to obsessions and compulsions: Theory, assessment, and treatment (pp. 91-105). Amsterdam, The Netherlands: Pergamon Elsevier.

Fu, A. S., \& Markus, H. R. (2014). My mother and me: Why tiger mothers motivate Asian American but Not European Americans. Personality and Social Psychology Bulletin, 40, 739-749. doi: 10.1177/ 0146167214524992

Gable, S. L., Reis, H. T., \& Elliot, A. J. (2000). Behavioral activation and inhibition in everyday life. Journal of Personality and Social Psychology, 78, 1135-1149. doi: 10.1037//0022-3514.78.6.1135

Gershoff, E. T. (2013). Spanking and child development: We know enough now to stop hitting our children. Child Development Perspectives, 7 , 133-137.

Gonzalez, M. G., Swanson, D. P., Lynch, M., \& Williams, G. C. (2014). Testing satisfaction of basic psychological needs as a mediator of the relationship between socioeconomic status and physical and mental health. Journal of Health Psychology. doi: 10.1177/ 1359105314543962

Greenspan, S. I. (1979). Intelligence and adaptation. New York, NY: International Universities Press.

Grolnick, W. S. (2002). The psychology of parental control: How well-meant parenting backfires. Adolescence, 37, 858-859.

Grolnick, W. S. (2012). The relations among parental power assertion, control, and structure: Commentary on Baumrind. Human Development, 55, 57-64. doi: 10.1159/000338533

Grolnick, W. S. (in press). Mothers' motivation for involvement in their children's schooling: Mechanisms and outcomes. Motivation and Emotion

Grolnick, W. S., Bridges, L., \& Connell, J. P. (1996). Emotional self-regulation in two-year-olds: Strategies and emotional expression in four contexts. Child Development, 67, 928-941. doi: 10.1111/ j.1467-8624.1996.tb01774.x

Grolnick, W. S., Bridges, L., \& Frodi, A. (1984). Maternal control style and the mastery motivation of one-year-olds. Infant Mental Health Journal, 5, 72-82.

Grolnick, W. S., Gurland, S. T., DeCourcey, W., \& Jacob, K. (2002) Antecedents and consequences of mothers' autonomy support: An experimental investigation. Developmental Psychology, 38, 143-155. doi: $10.1037 / / 0012-1649.38 .1 .143$

Grolnick, W. S., Kurowski, C. O., McMenamy, J. M., Rivkin, I., \& Bridges, L. J. (1998). Mothers' strategies for regulating their toddlers' distress. Infant Behavior and Development, 21, 437-450. doi 10.1016/S0163-6383(98)90018-2

Grolnick, W. S., \& Pomerantz, E. M. (2009). Issues and challenges in studying parental control: Toward a new conceptualization. Child Development Perspectives, 3, 165-170.

Grolnick, W. S., \& Ryan, R. M. (1987). Autonomy in children's learning: An experimental and individual difference investigation. Journal of Personality and Social Psychology, 52, 890-898. doi: 10.1037/ 0022-3514.52.5.890

Grolnick, W. S., \& Ryan, R. M. (1989). Parent styles associated with children's self-regulation and competence in school. Journal of Educational Psychology, 81, 143-154 
Grolnick, W. S., Ryan, R. M. \& Deci, E. L. (1991). The inner resources for school achievement: Motivational mediators of children's perception of their parents. Journal of Educational Psychology, 83, 508-517. doi: 10.1037/0022-0663.83.4.508

Grolnick, W. S., Weiss, L., McKenzie, L., \& Wrightman, J. (1996). Contextual, cognitive, and adolescent factors associated with parenting in adolescence. Journal of Youth and Adolescence, 25, 33-54. doi: 10.1007/BF01537379

Grolnick, W. S., Price, C. E., Beiswenger, K. L., \& Sauck, C. C. (2007). Evaluative pressure in mothers: Effects of situation, maternal, and child characteristics on autonomy supportive versus controlling behavior. Developmental Psychology, 43, 991-1002. doi: 10.1037/ 0012-1649.43.4.991

Gross, J. J. \& John, O. P. (2002). Wise emotion regulation. In L. F. Barrett \& P. Salovey (Eds.), The wisdom in feeling: Psychological processes in emotional intelligence. Emotions and social behavior. (pp. 297-319). New York, NY: Guilford Press

Sokol, B. W., Grouzet, F. M. E., \& Muller, U. (Eds.), (2013). Self-regulation and autonomy: Social, developmental, educational, and neurological dimensions of human contact. (pp. 191-207). New York, NY: Cambridge University Press.

Grusec, J. E., \& Goodnow, J. J. (1994). Impact of parental discipline methods on the child's internalization of values: A reconceptualization of current points of view. Developmental Psychology, 30, 4-19. doi: $10.1037 / 0012-1649.30 .1 .4$

Gurland, S. T., \& Grolnick, W. S. (2005). Perceived threat, controlling parenting, and children's achievement orientations. Motivation and Emotion, 29, 103-121. doi: 10.1007/s11031-005-7956-2

Harlow, H. F. (1950). Learning and satiation of response in intrinsically motivated complex puzzle performance by monkeys. Journal of Comparative and Physiological Psychology, 43, 289-294. DOI: $10.1037 / \mathrm{h} 0058114$

Harter, S. (2012). The construction of the self. New York, NY: Guilford.

Hartmann, H. (1964). On rational and irrational action. In Essays on ego psychology. New York, NY: International Universities Press. (Original work published 1947)

Hmel, B. A., \& Pincus, A. L. (2002). The meaning of autonomy: On and beyond the interpersonal circumplex. Journal of Personality, 70 , 277-310.

Hodgins, H. S., Koestner, R., \& Duncan, N. (1996). On the compatibility of autonomy and relatedness. Personality and Social Psychology Bulletin, 22, 227-237. doi: 10.1177/014616729622300

Hodgins, H. S., Brown, A. B., \& Carver, B. (2007). Autonomy and control motivation and self-esteem. Self \& Identity, 6, 189-208. doi: $10.1080 / 15298860601118769$

Hodgins, H. S., \& Knee, C. R. (2002). The integrating self and conscious experience. In E. L. Deci \& R. M. Ryan (Eds.), Handbook of self-determination research (pp. 87-100). Rochester, NY: University of Rochester Press.

Hodgins, H., Yacko, H., \& Gottlieb, E. (2006). Autonomy and nondefensiveness. Motivation and Emotion, 30, 283-293. doi: 10.1007/ s11031-006-9036-7

Hoffman, M. L. (1960). Power assertion by the parent and its impact on the child. Child Development, 31, 129-143.

Hooyman, A., Wulf, G., \& Lewthwaite, R. (2014). Impacts of autonomy-supportive versus controlling instructional language on motor learning. Human Movement Science, 36, 190-198. doi: 10.1080/ 02640414.2014.922693

Iyengar, S. S. \& DeVoe, S. E. (2003). Rethinking the value of choice: Considering cultural mediators of intrinsic motivation. In V. Murphy-Berman \& J. J. Berman (Eds.), Nebraska symposium on motivation: Cross-cultural differences in perspectives on self (Vol. 49, pp. 129-174). Lincoln, NE: University of Nebraska Press.

Jaffee, S. R., Moffitt, T. E., Caspi, A., \& Taylor, A. (2003). Life with (or without) father: The benefits of living with two biological parents depend on the father's antisocial behavior. Child Development, 74 (1), 109-126. doi: 10.1111/1467-8624.t01-1-00524

Jahoda, M. (1958). Current concepts of positive mental health. New York, NY: Basic Books.

Jang, H., Reeve, J., Ryan, R. M. \& Kim, A. (2009). Can self-determination theory explain what underlies the productive, satisfying learning experiences of collectivistically oriented Korean students? Journal of Educational Psychology, 101, 644-661. doi: 10.1037/a0014241

Jang, H., Kim, E. J., \& Reeve, J. (2012). Longitudinal test of selfdetermination theory's motivation mediation model in a naturally occurring classroom context. Journal of Educational Psychology, 104(4), 1175-1188. doi: 10.1037/a0028089

Jang, H., Reeve, J., \& Deci, E. L. (2010). Engaging students in learning activities: It's not autonomy support or structure, but autonomy support and structure. Journal of Educational Psychology, 102, 588-600. doi: 10.1037/a0019682

Jenike, M. A. (1991). Obsessive-compulsive disorder. In B. D. Beitman \& G. L. Klerman (Eds.), Integrating pharmacotherapy and psychotherapy (pp. 183-210). Washington, DC: American Psychiatric Association.

Johnson, J. G., Cohen, P., Chen, H., Kasen, S., \& Brook, J. S. (2006). Parenting behaviors associated with risk for offspring personality disorder during adulthood. Archives of General Psychiatry, 63(5), 579-587. doi: 10.1001/archpsyc.63.5.579.

Jordan, J. V. (1991). The relational self: A new perspective for understanding women's development. In J. Strauss \& G. R. Goethals (Eds.) The self: Interdisciplinary approaches (pp. 136-149).. New York, NY Springer-Verlag.

Joussemet, M., Mageau, G., \& Koestner, R. (2014). Promoting optimal parenting and children's mental health: A preliminary evaluation of the how-to parenting program. Journal of Child and Family Studies, 23, 949-964. DOI 10.1007/s10826-013-9751-0

Joussemet, M., Vitaro, F., Barker, E. D., Coté, S., Nagin, D., Zoccolillo, M., \& Tremblay, R. E. (2008). Controlling parenting and physical aggression during elementary school. Child Development, 79, 411-425. doi: $10.1111 /$ j.1467-8624.2007.01133.x

Kanat-Maymon, M., \& Assor, A. (2010). Perceived maternal control and responsiveness to distress as predictors of young adults' empathic responses. Personality and Social Psychology Bulletin, 36 (1), 33-46. doi: 10.1177/0146167209347381

Karavasilis, L., Doyle, A. B., \& Markiewicz, D. (2003). Association between parenting style and attachment to mother in middle childhood and adolescence. International Journal of Behavioral Development. 27(2), 153-164. doi: 10.1080/0165025024400015

Kasser, T. (2002). The high price of materialism. Cambridge, MA: MIT Press.

Kasser, T., \& Ryan, R. M. (1996) Further examining the American dream: Differential correlates of intrinsic and extrinsic goals. Personality and Social Psychology Bulletin, 22, 80-87. doi: 10.1177 0146167296223006

Kasser, T., Ryan, R. M., Zax, M., \& Sameroff, A. J. (1995). The relation of maternal and social environments to late adolescents' materialistic and prosocial values. Developmental Psychology, 31, 907-971. doi: 10.1037/0012-1649.31.6.907

Kasser, V. G., \& Ryan, R. M. (1999). The relation of psychological needs for autonomy and relatedness to vitality, well-being, and mortality in a nursing home. Journal of Applied Social Psychology, 29, 935-954. doi: 10.1111/j.1559-1816.1999.tb00133.x 
Katz, I , \& Assor, A. (2007) When choice motivates and when it does not. Educational Psychology Review, 19, 429-442. doi: 10.1007/ s10648-006-9027-y

Kawamura, K. Y., Frost, R. O., \& Harmatz, M. G. (2002). The relationship of perceived parenting styles to perfectionism. Personality \& Individual Differences, 32, 317-327. doi: 10.1016/S0191-8869(01)00026-5

Kennedy, S., Gogin, K., \& Nollen, N. (2004). Adherence to HIV medications: Utility of the theory of self-determination. Cognitive Therapy and Research, 28, 611-628. doi: 10.1023/B:COTR.0000045568. 95219 e 2

Kernis, M. H. (2003). Toward a conceptualization of optimal self-esteem. Psychological Inquiry, 14, 1-26. doi: 10.1207/S15327965PLI1401_01

Kim, S., Kochanska, G., Boldt, L. B., Nordling, J. K., \& O'Bleness, J. J. (2014). Developmental trajectory from early responses to transgressions to future antisocial behavior: Evidence for the role of the parent-child relationship from two longitudinal studies. Developmen and Psychopathology, 26(1), 93-109. doi: 10.1017/S0954579413000850

Knafo, A., \& Assor, A. (2007). Motivation for agreement with parenta values: Desirable when autonomous, problematic when controlled. Motivation and Emotion, 31, 232-245.

Kobak, R. Cassidy, J., \& Zir, Y (2004). Attachment-related trauma an post-traumatic stress disorder. In W. S. Rholes \& J. A. Simpson (Eds.), Adult attachment: theory research and clinical implication. (pp. 388-407). New York, NY: Guilford.

Koestner, R., \& McClelland, D. C. (1990). Perspectives on competence motivation. In L. Pervin (Ed.), Handbook of personality: Theory and research (pp. 527-548). New York, NY: Guilford.

Koestner, R., Bernieri, E., \& Zuckerman, M. (1992). Self-regulation an consistency between attitudes, traits, and behaviors. Personality and Social Psychology Bulletin, 18, 52-59. doi: 10.1177/0146167292181008

Koestner, R., Ryan, R. M., Bernieri, E., \& Holt, K. (1984). Setting limits on children's behavior: The differential effects of controlling versus informational styles on intrinsic motivation and creativity. Journal of Personality, 52, 233-248. doi: 10.1111/j.1467-6494.1984 .tb00879.x

Krapp, A. (2002). Structural and dynamic aspects of interest development: Theoretical considerations from an ontogenetic perspective. Learning and Instruction, 12, 383-409. doi: 10.1016/S0959-4752(01) 00011-1

Kroger, J., \& Marcia, J. E. (2011). The identity statuses: Origins, meanings and interpretations. In S. J. Schwartz, K. Luyckx, \& V. L. Vignoles (Eds.), Handbook of identity theory and research (pp. 31-54) New York, NY: Springer

Kusurkar, R. A., Ten Cate, T. J., Vos, C. M. P., Westers, P. \& Croiset, G. (2013). How motivation affects academic performance: A structura equation modelling analysis. Advances in Health Sciences Education, 18, 57-61. doi: 10.1007/s10459-012-9354-3

La Guardia, J. G., Ryan, R. M., Couchman, C. E., \& Deci, E. L. (2000). Within-person variation in security of attachment: A self-determination theory perspective on attachment, need fulfillment, and well-being. Journal of Personality and Social Psychology, 79, 367-384. doi: 10.1037//0022-3514.79.3.367

Lamb, M. E., \& Easterbrooks, M. A. (1981). Individual differences in parental sensitivity: Origins, components, and consequences. In M. E. Lamb \& L. R. Sherrod (Eds.), Infant social cognition: Empirical and theoretical considerations (pp. 127-154). Hillsdale, NJ: Erlbaum.

Landry, R., Whipple, N., Mageau, G. A., Joussemet, M., Gingras, I., Didio, L., \& Koestner, R. (2008). Trust in organismic development, autonomy support, and adaptation among mothers and their children: A self-determination theory approach to parenting. Motivation \& Emotion, 32, 173-188. doi: 10.1007/s11031-008-9092-2
Lee, W. \& Reeve, J. (2013). Self-determined, but not non-self-determined, motivation predicts activations in the anterior insular cortex: An fMRI study of personal agency. Social Cognitive Affective Neuroscience, 8 538-545. DOI: 10.1093/scan/nss029

Lekes, N., Gingras, I., Philippe, F. L., Koestner, R., \& Fang, J. Q. (2010) Parental autonomy-support, intrinsic life goals, and well-being among adolescents in China and north America. Journal of Youth and Adolescence, 39, 858-869. doi: 10.1007/s10964-009-9451-7

Lepper, M. R., Greene, D., \& Nisbett, R. E. (1973). Undermining children's intrinsic interest with extrinsic rewards: A test of the "overjustification" hypothesis. Journal of Personality and Social Psychology, 28 , 129-137. doi: 10.1037/h0035519

Levenson, R. W. (1999). The intrapersonal functions of emotion. Cognition and Emotion, 13, 481-504. doi: 10.1080/026999399379159

Linehan, M. (1993). Cognitive-behavioral treatment of borderline personality disorder. New York, NY: Guilford.

Loeber, R., \& Stouthammer-Loeber, M. (1986). Family factors as correlates and predictors of juvenile conduct disorders. In M. Tonry \& N. Morris (Eds.), Crime and justice (Vol. 7, pp. 29-149). Chicago, IL: University of Chicago Press.

Loevinger, J. (1976). Ego development. San Francisco: Jossey-Bass.

Luyckx, K., Vansteenkiste, M., Goossens, L., \& Duriez, B. (2009). Basic need satisfaction and identity formation: Bridging self-determination theory and process-oriented identity research. Journal of Counseling Psychology, 56, 276-288. doi: 10.1037/a0015349

Luyckx, K., Soenens, B., Vansteenkiste, M., Goossens, L., \& Berzonsky, M. D. (2007). Parental psychological control and dimensions of identity formation in emerging adulthood. Journal of Family Psychology, 21. 546-550. doi: 10.1037/0893-3200.21.3.546

Luyten, P., \& Blatt, S. J. (2013). Interpersonal relatedness and self-definition in normal and disrupted personality development: Retrospect and prospect. American Psychologist, 68, 172-183. doi $10.1037 / \mathrm{a} 0032243$

Lynch, M. F. (2013). Attachment, autonomy, and emotional reliance: A multilevel model. Journal of Counseling and Development, 91, 301-312. doi: 10.1002/j.1556-6676.2013.00098.x

Lynn, S. J, Lillienfeld, S. O., Merckelbach, H., Geisbrecht, T., \& van der Kloet, D. (2012). Dissociation and dissociative disorders: Challenging conventional wisdom. Current Directions in Psychological Science 21, $48-53$

Maccoby, E. E. (1980). Social development: Psychological growth and the parent-child relationship. New York, NY: Harcourt Brace Jovanovich

Mansour, S., Bruce, K. R., Steiger, H., Zuroff, D. C., Horowitz, S, Anestin, A. S., \& Sycz, L. (2012). Autonomous motivation: A predictor of treatment outcome in bulimia-spectrum eating disorders. Euro pean Eating Disorders Review, 20, 116-122. doi: 10.1002/erv.2154

Markland, D., Ryan, R. M., Tobin, V., \& Rollnick, S. (2005). Motivationa interviewing and self-determination theory. Journal of Social and Clinical Psychology, 24, 811-831. doi: 10.1521/jscp.2005.24.6.811

Markland, D., \& Tobin, V. J. (2010). Need support and behavioural regulations for exercise among exercise referral scheme clients: The mediating role of psychological need satisfaction. Psychology of Sport and Exercise, 11, 91-99. doi: 10.1016/j.psychsport.2009.07.001

Markus, H. R., Kitayama, S., \& Heiman, R. J. (1996). Culture an "basic" psychological principles. In E. T. Higgins \& A. W. Kruglansk (Eds.), Social psychology: Handbook of basic principles (pp. 857-913). New York, NY: Guilford.

Mask, L., \& Blanchard, C. M. (2011). The protective role of genera self-determination against the "thin ideal" media exposure on women's body image and eating-related concerns. Journal of Health Psychology, 16, 489-499. doi: 10.1177/1359105310385367 
Masterson, J. F. (1985). Treatment of the borderline adolescent: A developmental approach. New York, NY: Brunner/Mazel.

McCranie, E. W., \& Bass, J. D. (1984). Childhood family antecedents of dependency and self-criticism: Implications for depression. Journal of Abnormal Psychology, 93, 3-8. doi: 10.1037/0021-843X.93.1.3

McGraw, K. O., \& McCullers, J. C. (1979). Evidence of detrimental effect of extrinsic incentives on breaking a mental set. Journal of Experimental Social Psychology, 15, 285-294. doi: 10.1016/00221031(79)90039-8

Meissner, W. W. (1988). Treatment of patients in the borderline spectrum. Northvale, NJ: Aronson.

Miklikowska, M., Duriez, B., \& Soenens, B. (2011). Family roots of empathy-related characteristics: The role of perceived maternal and paternal need support in adolescence. Developmental Psychology, 47(5), 1342-1352. doi: 10.1037/a0024726

Mikulincer, M., \& Shaver, P. R. (2007). Attachment in adulthood: Structure, dynamics, and change. New York, NY: Guilford.

Miquelon, P., Vallerand, R. J., Grouzet, F. M. E., \& Cardinal, G. (2005) Perfectionism, academic motivation, and psychological adjustment: An integrative model. Personality and Social Psychology Bulletin, 31, 913-924. doi: 10.1177/0146167204272298

Miller, W. R., \& Rollnick, S. (2002). Motivational interviewing: Preparing people for change. New York, NY: Guilford Press.

Millon, T., Davis, R., Millon, C., Escovar, L., \& Meagher, S. (2000). Personality disorders in modern life. New York, NY: Wiley \& Sons, Inc.

Minuchin, S., Rosman, B., \& Baker, L. (1978). Psychosomatic families. Cambridge, MA: Harvard University Press.

Moller, A. C., Roth, G., Niemiec, C. P., \& Deci, E. L. (2014). Perceiving, selecting, and projecting. Manuscript under editorial review.

Mouratidis, A., Michou, A., Vansteenkiste, M., \& Lens, W. (2013). Perceived structure and achievement goals as predictors of students' self-regulated learning and affect and the mediating role of competence need satisfaction. Learning and Individual Differences, 23, 179-186. doi: 10.1016/j.lindif.2012.09.001

Mouratidis, A., Vansteenkiste, M., Sideridis, G., \& Lens, W. (2011). Variation in vitality and interest-enjoyment as a function of class-to-class need-supportive teaching and pupils' autonomous motivation. Journal of Educational Psychology, 103, 353-366. doi: 10.1037/a0022773

Muhtae, L., Zhou, Q., Eisenberg, N., \& Wang, Y. (2013). Predicting internalizing problems in Chinese children: The unique and interactive effects of parenting and child temperament. Development and Psychopathology, 25, 653-667. doi: 10.1017/S0954579413000084

Murayama, K., Matsumoto, M., Izuma, K., \& Matsumoto, K. (2010). Neural basis of the undermining effect of extrinsic reward on intrinsic motivation. Proceedings of the National Academy of Sciences of the United States of America, 107, 20911-20916. doi: 10.1073/ pnas. 1013305107

Murayama, K., Matsumoto, M., Izuma, K., Sugiura, A., Ryan, R. M., Deci, E. L., \& Matsumoto, K. (2014). How self-determined choice facilitates motivation and performance: A key role of the ventromedial prefrontal cortex. Cerebral Cortex, 25 (5), 1241-1251. doi: medial prefrontal cor

Muraven, M., Gagné, M., \& Rosman, H. (2008). Helpful self-control: Autonomy support, vitality, and depletion. Journal of Experimental Social Psychology, 44, 573-585.

Neyrinck, B., Vansteenkiste, M., Lens, W., Hutsebaut, D., \& Duriez, B. (2006). Cognitive, affective and behavioral correlates of internalization of regulations for religious activities. Motivation and Emotion, 30, 321-332. doi: 10.1007/s11031-006-9048-3

Ng, Y., Ntoumanis, N., Thøgersen-Ntoumani, C., Deci, E. L., Ryan, R. M., Duda, J., \& Williams, G. C. (2012). Self-determination theory applied to health contexts: A meta-analysis. Perspectives on Psychological Science, 7, 325-340. doi: 10.1177/1745691612447309

Noom, M. J., Dekovic, M., \& Meeus, W. H. J. (1999). Autonomy, attachment and psychosocial adjustment during adolescence: A double-edged sword? Journal of Adolescence, 22, 771-783. doi: 10.1006/jado. 1999.0269

Othmer, E., \& Othmer, S. C. (2002). The clinical interview using DSM-IV-TR: Vols. 1 and 2. Washington, DC: American Psychiatric Publishing, Inc.

O’Rorke, D. J., Smith, R. E., Smoll, F. L., \& Cumming, S. P. (2012). Parent-initiated motivational climate and young athletes' intrinsicextrinsic motivation: Cross sectional and longitudinal relations. Journal of Child and Adolescent Behavior, 1, 109. doi: 10.4172/jcalb. 1000109

Parrott, W. G. (1993). Beyond hedonism: Motives for inhibiting good moods and for maintaining bad moods. In D. M. Wegner \& J. W. Pennebaker (Eds.), Handbook of mental control. Century psychology series (pp. 278-305). Upper Saddle River, NJ: Prentice-Hall, Inc.

Patall, E. A., Cooper, H., \& Robinson, C. (2008). The effects of choice on intrinsic motivation and related outcomes: A meta-analysis of research findings. Psychological Bulletin, 134, 270-300. doi: 10.1037/ 0033-2909.134.2.270

Patall, E. A., Dent, A. L., Oyer, M., \& Wynn, S. R. (2012). Student autonomy and course value: The unique and cumulative roles of various teacher practices. Motivation and Emotion, 37 (1), 14-32. DOI: 10.1007/s11031-012-9305-6

Pelletier, L., Dion, S. C., \& Levesque, C. (2004). Can self-determination help protect women against socio-cultural influences about body image and reduce their risk of experiencing bulimic symptoms? Journal of Social and Clinical Psychology, 23, 61-88. doi: 10.1521/ jscp.23.1.61.26990

Pfander, A. (1967). Phenomenology of willing and motivation (H. Spiegelberg, Trans.). Evanston, IL: Northwestern University Press. (Original work published 1908)

Piaget, J. (1971). Biology and knowledge. Chicago, Ill: University of Chicago Press.

Plant, R., \& Ryan, R. M. (1985). Intrinsic motivation and the effects of self-consciousness, self-awareness, and ego-involvement: An investigation of internally controlling styles. Journal of Personality. 53. 435-449. doi: 10.1111/j.1467-6494.1985.tb00375.x

Pomerantz, E. M., \& Eaton, M. M. (2001). Maternal intrusive support in the academic context: Transactional socialization processes. Developmental Psychology, 37(2), 174-186. doi: 10.1037//0012-1649.37.2.174

Pulfrey, C., Buchs,C., \& Butera, F. (2012). Why grades engender performance-avoidance goals: The mediating role of autonomous motivation. Journal of Educational Psychology, 103, 683-700. doi: 10.1037/a0023911

Raine, A. (2013). The anatomy of violence. New York, NY: Vintage Books

Reeve, J., \& Halusic, M. (2009). How K-12 teachers can put selfdetermination theory principles into practice. Theory and Research in Education, 7, 145-154

Reeve, J., \& Jang, H. (2006). What teachers say and do to support students' autonomy during a learning activity. Journal of Educational Psychology, 98, 209-218. doi: 10.1037/0022-0663.98.1.209

Rogers, C. (1963). The actualizing tendency in relation to "motives" and to consciousness. In M. R. Jones (Ed.), Nebraska symposium on motivation (Vol. 11, pp. 1-24). Lincoln, NE: University of Nebraska Press.

Roth, G., \& Assor, A. (2010). Parental conditional regard as a predictor of deficiencies in young children's capacities to respond to sad feelings. Infant and Child Development, 19, 465-477. doi: 10.1002/icd.676 
Roth, G., \& Assor, A. (2012). The costs of parental pressure to expres emotions: Conditional regard and autonomy support as predictors of emotion regulation and intimacy. Journal of Adolescence, 35, 799-808 doi: 10.1016/j.adolescence.2011.11.005

Roth, G., Benita, M., Amrani, C., Shachar, B. H., Asoulin, H., Moed, A., Bibi, U., \& Kanat-Maymon, Y. (in press). Integration of negative emotional experience versus suppression: Addressing the question of adaptive functioning. Emotion.

Roth, G., Assor, A., Niemiec, C. P., Ryan, R. M., \& Deci, E. L. (2009). The emotional and academic consequences of parental conditional regard: Comparing conditional positive regard, conditional negative regard, and autonomy support as parenting practices. Developmental Psychology, 45, 1119-1142.

Roth, G., Ron, T., \& Benita, M. (2009). Mothers' parenting practices and adolescents' learning from their mistakes in class: The mediating role of adolescent's self-disclosure. Learning and Instruction , 19, 506-512 doi: 10.1016/j.learninstruc.2008.10.001

Ryan, R. M. (1982). Control and information in the intrapersonal sphere: An extension of cognitive evaluation theory. Journal of Personality and Social Psychology, 43, 450-461. doi: 10.1037//0022-3514.43.3.450

Ryan, R. M. (1993). Agency and organization: Intrinsic motivation, autonomy and the self in psychological development. In J. Jacob (Ed.), Nebraska Symposium on Motivation: Vol. 40. Developmental perspectives on motivation (pp. 1-56). Lincoln, NE: University of Nebraska Press.

Ryan, R. M. (1995). Psychological needs and the facilitation of integrative processes. Journal of Personality, 63, 397-427. doi: 10.1111/ j.1467-6494.1995.tb00501.x

Ryan, R. M. (2005). The developmental line of autonomy in the etiology, dynamics, and treatment of borderline personality disorders. Development and Psychopathology, 17, 987-1006. doi: 10.1017/ ders. Development and

Ryan, R. M., Brown, K. W., \& Creswell, J. D. (2007). How integrative is attachment theory? Unpacking the meaning and significance of felt security. Psychological Inquiry, 18, 1-6.

Ryan, R. M., \& Connell, J. P. (1989). Perceived locus of causality and internalization: Examining reasons for acting in two domains. Journal of Personality and Social Psychology, 57, 749-761. do 10.1037/0022-3514.57.5.749

Ryan, R. M., Connell, J. P., \& Deci, E. L. (1985). A motivational analysi of self-determination and self-regulation in education. In C. Ames \& R. E. Ames (Eds.), Research on motivation in education: The classroom milieu (pp. 13-51). New York, NY: Academic Press.

Ryan, R. M., Curren, R. R., \& Deci, E. L. (2013). What humans need: Flourishing in Aristotelian philosophy and self-determination theory. In A. S. Waterman (Ed.), The best within us: Positive psychology perspectives on eudaimonic functioning (pp. 57-75). Washington, DC: American Psychological Association Books.

Ryan, R. M., \& Deci, E. L. (2000a). The darker and brighter sides of human existence: Basic psychological needs as a unifying concept. Psychological Inquiry, 11, 319-338. doi: 10.1207/ fying concept. Psych 15327965 PLI1104_03

Ryan, R. M., \& Deci, E. L. (2000b). Self-determination theory and the facilitation of intrinsic motivation, social development, an well-being. American Psychologist, 55, 68-78. doi: 10.1037//0003066X.55.1.68

Ryan, R. M., \& Deci, E. L. (2008). A self-determination approach to psychotherapy: The motivational basis for effective change. Canadia Psychology, 49, 186-193. doi: 10.1037/a0012753

Ryan, R. M., \& Deci, E. L. (2013). Motivation and development in the context of schools: Research on how classroom environments affect student engagement, well-being, and performance. In B. Sokol,
F. M. E. Grouzet, \& U. Muller (Eds.), Self-regulation and autonomy: Social, developmental, educational, and neurological dimensions of human contact (pp. 191-207). New York, NY: Cambridge University Press.

Ryan, R. M., \& Deci, E. L. (2011). Multiple identities within a single self: A self-determination theory perspective on internalization within contexts and cultures. In M. R. Leary \& J. P. Tangney (Eds.), Handbook of self and identity (pp. 225-246). New York, NY: Guilford.

Ryan, R. M., Deci, E. L., Grolnick, W. S., \& La Guardia, J. G. (2006) The significance of autonomy and autonomy support in psychological development and psychopathology. In D. Cicchetti \& D. Cohen (Eds.), Developmental psychopathology: Volume 1, theory and methods (2nd ed., pp. 795-849). New York, NY: John Wiley \& Sons.

Ryan, R. M., \& Grolnick, W. S. (1986). Origins and pawns in the classroom: Self-report and projective assessments of individual differences in children's perceptions. Journal of Personality and Social Psychology, 50, 550-558. doi: $10.1037 / / 0022-3514.50 .3 .550$

Ryan, R. M., \& Hawley, P. (in press). Naturally good? Basic psychological needs and the proximal and evolutionary bases of human benevolence. In M. Leary \& K. W. Brown (Eds.), The psychology of hypoegoic behavior. New York, NY: Guilford.

Ryan, R. M., \& Kuczkowski, R. (1994). Egocentrism and heteronomy A study of imaginary audience, self-consciousness, and public individ uation in adolescence. Journal of Personality, 62, 219-238.

Ryan, R. M., Kuhl, J. \& Deci, E. L. (1997). Nature and autonomy: Organizational view of social and neurobiological aspects of self-regulation in behavior and development. Development and Psychopathology, 9, 701-728.

Ryan, R. M., La Guardia, J. G., Solky-Butzel, J., Chirkov, V., \& Kim. Y. (2005). On the interpersonal regulation of emotions: Emotional reliance across gender, relationships, and cultures. Personal Relationships, 12, 145-163. doi: 10.1111/j.1350-4126.2005.00106.x

Ryan, R. M., Legate, N., Niemiec, C. P., \& Deci, E. L. (2012). Beyond illusions and defense: Exploring the possibilities and limits of human autonomy and responsibility through self-determination theory. In P. R. Shaver \& M. Mikulincer (Eds.), Meaning, mortality, and choice (pp. 215-233). Washington, DC: American Psychological Association.

Ryan, R. M., \& Lynch, J. H. (1989). Emotional autonomy versus detachment: Revisiting the vicissitudes of adolescence and young adult hood. Child Development, 60, 340-356. doi: 10.1111/j.1467-8624. 1989.tb02720.x

Ryan, R. M., Lynch, M. L., Vansteenkiste, M., \& Deci, E. L. (2011). Motivation and autonomy in counseling, psychotherapy, and behavior change: A look at theory and practice. Counseling Psychologist, 39 , 193-260. doi: 10.1177/0011000009359313

Ryan, R. M., Mims, V., \& Koestner, R. (1983). Relation of reward contingency and interpersonal context to intrinsic motivation: A review and test using cognitive evaluation theory. Journal of Personality and Social Psychology, 45, 736-750. doi: 10.1037/0022-3514.45.4.736

Ryan, R. M., Patrick, H., Deci, E. L., \& Williams, G. C. (2008). Facilitating health behavior change and its maintenance: Interventions base on self-determination theory. European Health Psychologist, 10, 2-5.

Ryan, R. M., Plant, R. W., \& O'Malley, S. (1993). Initial motivations for alcohol treatment: Relations with patient characteristics, treatment involvement and dropout. Addictive Behaviors, 20, 279-297. doi: 10.1016/0306-4603(94)00072-7

Ryan, R. M., Rigby, S., \& King, K. (1993). Two types of religious internalization and their relations to religious orientations and mental health. Journal of Personality and Social Psychology, 65, 586-596. doi: 10.1037/0022-3514.65.3.586

Ryan, R. M., Sheldon, K. M., Kasser, T., \& Deci, E. L. (1996). All goals are not created equal: An organismic perspective on the nature of 
goals and their regulation. In P. M. Gollwitzer \& J. A. Bargh (Eds.), The psychology of action: Linking cognition and motivation to behavior (pp. 7-26). New York, NY: Guilford.

Ryan, R. M., Stiller, J. D., \& Lynch, J. H. (1994). Representations of relationships to teachers, parents, and friends as predictors of academic motivation and self-esteem. Journal of Early Adolescence, 14, 226-249.

Savard, A., Joussemet, M., Pelletier, J. E., \& Mageau, G. A. (2013). The benefits of autonomy support for adolescents with severe emotional and behavioral problems. Motivation and Emotion, 37, 688-700. doi: 10.1007/s11031-013-9351-8

Schaefer, E. S. (1959). A circumplex model for maternal behavior. Journal of Abnormal and Social Psychology, 59, 226-235. doi: 10.1037 h0041114

Schafer, R. (1968). Aspects of internalization. New York, NY: International Universities Press.

Sears, R. R., Maccoby, E., \& Levin, H. (1957). Patterns of child rearing. Evanston, IL: Row, Peterson.

Shapiro, D. (1981). Autonomy and rigid character. New York, NY: Basic Books.

Shields, A., Cicchetti, D., \& Ryan, R. M. (1994). The development of emotional and behavioral self-regulation and social competence among maltreated school-age children. Development and Psychopathology, 6 , maltrea

Shields, A., Ryan, R. M., \& Cicchetti, D. (2001). Narrative representations of caregivers and emotion dysregulation as predictors of maltreated children's rejection by peers. Developmental Psychology, 37, 321-337. doi: 10.1037//0012-1649.37.3.321

Silk, K. R., Lee, S., \& Hill, E. M. (1995). Borderline personality disorder symptoms and severity of sexual abuse. American Journal of Psychiatry, 152, 1059-1064.

Silk, J. S., Morris, A. S., Kanaya, T., \& Steinberg, L. (2003). Psychological control and autonomy granting: Opposite ends of a continuum or distinct constructs? Journal of Research on Adolescence, 13, 113-128. doi: $10.1111 / 1532-7795.1301004$

Sierens, E., Vansteenkiste, M., Goossens, L., Soenens, B., \& Dochy, F. (2009). The synergistic relationship of perceived autonomy support and structure in the prediction of self-regulated learning. British Journal of Educational Psychology, 79, 57-68. doi: 10.1348/ 000709908 X304398

Skinner, B. F. (1953). Science and human behavior. New York, NY: Macmillan

Smetana, J. G., Campione-Barr, N., \& Daddis, C. (2004). Longitudinal development of family decision making: Defining healthy behavioral autonomy for middle-class African American adolescents. Child Development, 75, 1418-1434. doi: 10.1111/j.1467-8624. 2004.00749.x

Sobral, D. T. (2004). What kind of motivation drives medical students' learning quests? Medical Education, 38(9), 950-957. doi: 10.1111/ j.1365-2929.2004.01913.x

Soenens, B., Berzonsky, M. D., Dunkel, C., Papini, D., \& Vansteenkiste, M. (2011). Are all identity commitments created equally? The importance of motives for late adolescents' personal adjustment. International Journal of Behavioral Development, 35, 358-369. doi: 10.1177/ 016502541140595

Soenens, B., Elliot, A. J., Goossens, L., Vansteenkiste, M., Luyten, P., \& Duriez, B. (2005). The intergenerational transmission of perfectionism: Parents' psychological control as intervening variable. Journal of Family Psychology, 19, 358-366. doi: 10.1037/0893-3200.19.3.358

Soenens, B., Luyckx, K., Vansteenkiste, M., Luyten, P., Duriez, B., \& Goossens, L. (2008). Maladaptive perfectionism as an intervening variable between psychological control and adolescent depressive symptoms: A three-wave longitudinal study. Journal of Family Psychology, 22, 465-474. doi: 10.1037/0893-3200.22.3.465

Soenens, B., Park, S.-Y., Vansteenkiste, M., \& Mouratidis, A. (2012) Perceived parental psychological control and adolescent depressive experiences: A cross-cultural study with Belgian and South-Korean adolescents. Journal of Youth and Adolescence, 35, 261-272 doi 10.1016/j.adolescence.2011.05.001

Soenens, B., \& Vansteenkiste, M. (2005). Antecedents and outcomes of self-determination in three life domains: The role of parents' an teachers' autonomy support. Journal of Youth and Adolescence, 34, 589-604 doi: 10.1007/s10964-005-8948-y

Soenens, B., \& Vansteenkiste, M. (2010). A theoretical upgrade of the concept of parental psychological control: Proposing new insights on the basis of self-determination theory. Developmental Review, 30, 74-99. doi: 10.1016/j.dr.2009.11.001

Soenens, B., \& Vansteenkiste, M. (2011). When is identity congruent with the self? A self-determination perspective. In S. J. Schwartz, K. Luyckx, \& V. L. Vignoles (Eds.), Handbook of identity theory and research (pp. 381-402). New York, NY: Springer.

Soenens, B., Vansteenkiste, M., Duriez, B., \& Goossens, L. (2006). In search of the sources of psychologically controlling parenting: The role of parental separation anxiety and parental maladaptive perfectionism. Journal of Research on Adolescence, 16, 539-559. doi: 10.1111/j.1532-7795.2006.00507.x

Soenens, B., Vansteenkiste, M., Duriez, B, Luyten, P. \& Goossens, L. (2005). Maladaptive perfectionistic self-representations: The mediational link between psychological control and adjustment. Personality and Individual Differences, 38, 487-498. doi: 10.1016/j.paid. 2004.05.008

Soenens, B., Vansteenkiste, M., Goossens, L., Duriez, B., \& Niemiec, C. (2008). The intervening role of relational aggression between psychological control and friendship quality. Social Development, 17, 561-581. doi: 10.1111/j.1467-9507.2007.00454.x

Soenens, B., Vansteenkiste, M., Lens, W., Luyckx, K., Goossens, L., Beyers, W., \& Ryan, R. M. (2007). Conceptualizing parental autonomy support: Adolescent perceptions of promotion of independence versus promotion of volitional functioning. Developmental Psychology, sus promotion of volitional functioning. Deve

Soenens, B., Vansteenkiste, M., \& Sierens, E. (2009). How are parenta psychological control and autonomy support related? A clusteranalytic approach. Journal of Marriage and Family, 71, 187-202. doi: $10.1111 / j .1741-3737.2008 .00589$

Soenens, B., Vansteenkiste, M., Vandereycken, W., Luyten, P., Sierens, E. \& Goossens, L. (2008). Perceived parental psychological control and eating disordered symptoms: Maladaptive perfectionism as a possible intervening variable. Journal of Nervous and Mental Disease, 196 , 144-152. doi: 10.1097/NMD.0b013e318162aabf

Soenens, B., Vansteenkiste, M., \& Van Petegem, S. (in press). Let's not throw out the baby with the bathwater: Applying the principle of "universalism without uniformity" to autonomy-supportive and controlling parenting. Child Development Perspectives.

Sperry, L. (2003). Handbook of diagnosis and treatment of DSM-IV-TR personality disorders. New York, NY: Brunner-Routledge

Steinberg, L. (2005). Cognitive and affective development in adolescence. Trends in Cognitive Science, 9, (2), 69-74. doi: 10.1016/j.tics. 2004.12.005

Steinberg, L., \& Silverberg, S. (1986). The vicissitudes of autonomy in adolescence. Child Development, 57, 841-851.

Steinberg, M., \& Schnall, M. (2001). The stranger in the mirror. New York, NY: Cliff Street Books.

Stern, D. N. (1985). The interpersonal world of the infant. New York, NY: Basic Books. 
Stevenson, M. B. \& Lamb, M. E. (1981). The effects of social experience and social style on cognitive competence and performance. In M. E. Lamb \& L. R. Sherrod (Eds.), Infant social cognition (pp. 375-394). Hillsdale, NJ: Erlbaum.

Strauss, J., \& Ryan, R. M. (1987). Autonomy disturbances in subtypes of anorexia nervosa. Journal of Abnormal Psychology, 96, 254-258. doi 10.1037//0021-843X.96.3.254

Stipek, D., \& Seal, K. (2001) Motivated minds: Raising children to love learning. New York, NY: Henry Holt.

Strober, M., \& Humphrey, L. L. (1987). Familial contributions to the etiology and course of anorexia and bulimia. Journal of Consulting and Clinical Psychology, 55, 654-659.

Talwar, V., \& Lee, K. (2011). A punitive environment fosters children' dishonesty: A natural experiment. Child Development, 82, 1751-1758. doi: 10.1111/j.1467-8624.2011.01663.x

Thrash, T. M., \& Elliot, A. J. (2002). Implicit and self-attributed achievement motives: Concordance and predictive validity. Journal of Personality, 70, 729-756.

Tsai, Y., Kunter, M., Lüdtke, O., Trautwein, U., \& Ryan, R. M. (2008). What makes lessons interesting? The role of situational and individual factors in three school subjects. Journal of Educational Psychology, 100, 460-472. doi: 10.1037/0022-0663.100.2.460

Twenge, J., Gentile, B., DeWall, C. N., Ma, D., Lacefield, K., \& Schurtz, D. R. (2010). Birth cohort increases in psychopathology amon young Americans, 1938-2007: A cross-temporal meta-analysis of the MMPI. Clinical Psychology Review, 30, 145-154. doi: 10.1016 j.cpr.2009.10.005

Vallerand, R. J. (2010). On passion for life activities: The dualistic mode of passion. In P. Devine \& A. Plant (Eds.) Advances in Experimental Social Psychology (Vol. 42, pp. 97-193). London, UK: Elsevier.

Vallerand, R. J., O'Connor, B. P., \& Hamel, M. (1995). Motivation in later life: Theory and assessment. International Journal of Aging and Human Development, 41, 221-238. doi: 10.2190/YLFMDGUE-HRL2-VWLG

Van den Broeck, A., Schreurs, B., De Witte, H., Vansteenkiste, M., Germeys, F., \& Schaufeli, W. (2011). Understanding workaholics' motivations: A self-determination perspective. Applied Psychology: An International Review, 60(4), 600-621. doi: 10.1111/j.1464 0597.2011.00449.x

Vandereycken, W., \& Vansteenkiste, M. (2009). Let eating disorde patients decide: Providing choice might reduce early drop-out from inpatient treatment. European Eating Disorders Review, 17, 177-183. doi: $10.1002 /$ erv.917

Van der Hart, O. Nijenhuis, E., \& Steele, K. (2006). The haunted self: Structural dissociation and the treatment of chronic traumatization. New York, NY: Norton.

Van der Kaap-Deeder, J., Vansteenkiste, M., Soenens, B., Verstuyf, J., Boone, L., \& Smets, J. (in press). Fostering self-endorsed motivation to change in patients with an eating disorder: The role of perceived autonomy support and psychological need satisfaction. International Journal of Eating Disorders.

Van Petegem, S., Beyers, W., Vansteenkiste, M., \& Soenens, B. (2012). On the association between adolescent autonomy and psychosocial functioning: Examining decisional independence from self-determination theory perspective. Developmental Psychology, 48 , 76-88. doi: 10.1037/a0025307

Van Petegem, S., Vansteenkiste, M., \& Beyers, W. (2013). The jingle-jangle fallacy in adolescent autonomy in the family: In search of an underlying structure. Journal of Youth and Adolescence, 42, 994-1014. doi 10.1007/s10964-012-9847-7
Van Petegem, S., Soenens, B., Vansteenkiste, M., \& Beyers, W. (in press) Rebels with a cause? Adolescent defiance from the perspective of reactance theory and self-determination theory. Child Development.

Vansteenkiste, M., Niemiec, C., \& Soenens, B. (2010).The development of the five mini-theories of self-determination theory: An historical overview, emerging trends, and future directions. In T. Urdan \& S. Karabenick (Eds.), Advances in motivation and achievement, vol. 16: The decade ahead. Bingley, UK: Emerald Publishing.

Vansteenkiste, M., Sierens, E., Soenens, B., Goossens, L., Dochy, F., Aelterman, N., ..., Beyers, W. (2012). Identifying configurations of perceived teacher autonomy support and structure: Associations with self-regulated learning, motivation and problem behavior. Learning and Instruction, 22, 431-439. doi: 10.1016/j.learninstruc.2012.04.002

Vansteenkiste, M., Simons, J., Lens, W., Soenens, B., \& Matos, L. (2005) Examining the motivational impact of intrinsic versus extrinsic goal framing and autonomy-supportive versus internally controlling communication style on early adolescents' academic achievement. Child Development, 76, 483-501. doi: 10.1111/j.1467-8624.2005.00858.x

Vansteenkiste, M., Soenens, B., \& Vandereycken, W. (2005). Motivation to change in eating disorder patients: A conceptual clarification on the basis of self-determination theory. International Journal of Eating Disorders, 37, 207-219. doi: 10.1002/eat.20099

Vansteenkiste, M., Soenens, B., Van Petegem, S., \& Duriez, B. (2014) Longitudinal associations between adolescent perceived degree and style of parental prohibition and internalization and defiance. Developmental Psychology, 50, 229-236. doi: 10.1037/a0032972

Vansteenkiste, M., \& Ryan, R. M. (2013). On psychological growth and vulnerability: Basic psychological need satisfaction and need frustration as a unifying principle. Journal of Psychotherapy Integration, 23 , 263-280. doi: 10.1037/a0032359

Verschueren, K., Doumen, S., Vervoort, E., \& Vansteenkiste, M. (2013). How early parent-child interactions are carried forward into elementary school: Relational and motivational pathway to academic success. Manuscript submitted for publication.

Verstuyf, J., Vansteenkiste, M., \& Soenens, B. (2012). Eating regulation and bulimic symptoms: The differential correlates of health-focused and appearance-focused eating regulation. Body Image: An International Research Journal, 9, 108-117. doi: 10.1016/j.bodyim.201 .09 .003

Verstuyf, J, Vansteenkiste, M., Soenens, B., Boone, L. \& Mouratidis, A. (2013). Daily ups-and-downs binge eating symptoms: The role of need satisfaction, self-control and emotional eating style. Journal of Social and Clinical Psychology, 32, 335-361.

Vignoles, V. L., Schwartz, J., \& Luyckx, K. (2011). Introduction: Towards an integrative view of identity. In S. J. Schwartz, K. Luyckx, \& V. L. Vignoles (Eds.), Handbook of identity theory and research (pp. 1-30). New York, NY: Springer.

Warneken, F., \& Tomassell, M. (2008). Extrinsic rewards undermine altruistic tendencies in 20-month olds. Developmental Psychology, 44, 1785-1788 doi: $10.1037 / \mathrm{a} 0013860$

Waters, E., Wippman, J., \& Sroufe, L. A. (1979). Attachment, posi tive affect, and competence in the peer group: Two studies in construct validation. Child Development, 50, 821-829. doi: 10.1111/j.14678624.1979.tb02431.x

Weinstein, N., Deci, E. L., \& Ryan, R. M. (2011). Motivational determinants of integrating positive and negative past identities. Journal of Personality and Social Psychology, 100, 527-544. doi: 10.1037 a 0022150

Weinstein, N. \& Hodgins, H. S. (2009). The moderating role of autonomy and control on the benefits of written emotion expression. Personality and Social Psychology Bulletin, 35, 351-364. doi: $10.1177 / 0146167208328165$ 
Weinstein, N., Hodgins, H. S., \& Ostvik-White, E. (2011). Humor as aggression: Effects of motivation on hostility expressed in humor appreciation. Journal of Personality and Social Psychology, 100, 1043-1055. doi: 10.1037/a0022495

Weinstein, N., Przybylski, A. K., \& Ryan, R. M. (2012). The index of autonomous functioning: Development of a scale of human autonomy. Journal of Research in Personality, 46, 397-413. DOI: 10.1016/j.jrp.2012.03.007

Weinstein, N., Przybylski, A. K., \& Ryan, R. M. (2013). The integrative process: New research and future directions. Current Directions in Psychological Science, 22, 69-74.

Weinstein, N., \& Ryan, R. M. (2010). When helping helps: Autonomous motivation for prosocial behavior and its influence on well-being for the helper and recipient. Journal of Personality and Social Psychology, 98, 222-244. doi: 10.1037/a0016984

Weinstein, N., Ryan, W. S., DeHaan, C. R., Przybylski, A. K., Legate, N., \& Ryan, R. M. (2012). Parental autonomy support and discrepancies between implicit and explicit sexual identities: Dynamics of self-acceptance and defense. Journal of Personality and Social Psychology, 102, 815-832. doi: 10.1037/a0026854

Weiss, L. A., \& Grolnick, W. S. (1991, April). The roles of parental involvement and support for autonomy in adolescent symptomatology. Paper presented at the biennial meeting of the Society for Research in Child Development, Seattle, WA

Werner, H. (1948). Comparative psychology of mental development. New York, NY: International Universities Press.

Westen, D. (1991). Cognitive-behavioral interventions in the psychoanalytic psychotherapy of borderline personality disorders. Clinical Psychology Review, 11, 211-230. doi: 10.1016/0272-7358(91)90101-Y

Whiffin, V. E., \& Sassville, T. M. (1991). Dependency, self-criticism, and recollections of parenting: Sex differences and the role of depressive affect. Journal of Social and Clinical Psychology, 10, 121-133. doi 10.1521/jscp.1991.10.2.121

Whipple, N., Bernier, A., \& Mageau, G. A. (2009). Attending to the exploration side of infant attachment: Contributions from self-determination theory. Canadian Psychology, 50, 219-229 doi: 10.1037/a0016322

Whipple, N., Bernier, A., \& Mageau, G. A. (2011). Broadening the study of infant security of attachment: Maternal autonomy-support in the context of infant exploration. Social Development, 20(1), 17-32. DOI: context of infant exploration. Sociel
Williams, G. C. Grow, V. M., Ryan, R. M., Friedman, Z \& \& Deci, E. L. (1996). Motivational predictors of weight loss and weight-loss maintenance. Journal of Personality and Social Psychology, 70, 115-126. DOI 10.1037/0022-3514.70.1.115

Williams, G. C., McGregor, H. A., Sharp, D., Levesque, C., Kouides, R. W., Ryan, R. M., \& Deci, E. L. (2006). Testing a self-determination theory intervention for motivating tobacco cessation: Supporting autonomy and competence in a clinical trial. Health Psychology, 25, 91-101. doi: 10.1037/0278-6133.25.1.91

Williams, G. C., Rodin, G. C., Ryan, R. M., Grolnick, W. S., \& Deci, E. L. (1998). Autonomous regulation and long-term medication adherence in adult outpatients. Health Psychology, 17, 269-276. doi: 10.1037/0278-6133.17.3.269

Wuyts, D., Vansteenkiste, M., Soenens, B., \& Assor, A. (in press). An examination of the dynamics involved in child-invested parental contingent self-esteem. Parenting: Science and Practice.

Winnicott, D. W. (1965). The maturational process and the facilitating environment. New York, NY: International Universities Press. (Original work published 1960)

Yamauchi, H., \& Tanaka, K. (1998). Relations of autonomy, selfreferenced beliefs and self-regulated learning among Japanese children. Psychological Reports, 82, 803-816. doi: 10.2466/PR0 82.3.803-816

Yarrow, L. J., Rubenstein, J. C. \& Pedersen, F. A. (1975). Infant and environment: Early cognitive and motivational development. Washington, DC: Wiley.

Zanarini, M. C. (Ed.). (1997). Role of sexual abuse in the etiology of borderline personality disorder. Washington, DC: American Psychiatric Association.

Zeldman, A., Ryan, R. M., \& Fiscella, K. (2004) Client motivation, autonomy support and entity beliefs: Their role in methadone maintenance treatment. Journal of Social and Clinical Psychology, 23, 675-696.

Zuroff, D. C., Koestner, R., Moskowitz, D. S., McBride, C., \& Bagby, R. M. (2012). Therapist's autonomy support and patient's self-criticism predict motivation during brief treatments for depression. Journal of Social and Clinical Psychology, 31 (9), 903-932.

Zuroff, D. C., Koestner, R., Moskowitz, D. S., McBride, C., Marshall, M., \& Bagby, R. M. (2007). Autonomous motivation for therapy: A new common factor in brief treatments for depression. Psychotherapy Research, 17, 137-148. doi: 10.1080/10503300600919380 


\section{Queries in Chapter 9}

Q1. We have shortened running head, if it exceeds the text width. Please confirm if this OK. 